\title{
Explainable Data Fusion
}

\author{
A Thesis presented to \\ the Faculty of the Graduate School \\ at the University of Missouri
}

In Partial Fulfillment

of the Requirements for the Degree

Doctor of Philosophy

by

Bryce J. Murray

Dr. Derek Anderson, Thesis Supervisor

May 2021 
The undersigned, appointed by the Dean of the Graduate School, have examined the dissertation entitled:

\section{Explainable Data Fusion}

presented by Bryce J. Murray, a candidate for the degree of Doctor of Philosophy and hereby certify that, in their opinion, it is worthy of acceptance.

\begin{tabular}{c}
\hline Dr. Derek T. Anderson \\
\hline Dr. James M. Keller \\
\hline Dr. Grant J. Scott \\
\hline Dr. Curt H. Davis \\
\hline
\end{tabular}

Dr. Mihail Popescu 


\section{ACKNOWLEDGMENTS}

There are several people I would like to thank and acknowledge throughout this academic journey. The first is my beautiful wife, Kate. She graciously agreed to Missouri with me despite not knowing anyone or anything about this area. Columbia has become our first home where we've grown leaps and bounds from the newlyweds we once were (especially as we get ready to welcome Margaret in the next few months). Pursuing a doctorate can be very time intensive, and I want to acknowledge and thank her for the flexibility she has shown in doing life together. Next, I'd like to thank my parents who have always supported me in whatever I was doing. From little league baseball games to $20+$ years of school, they have always believed and been

there for whatever I needed. Accomplishing anything without a support system is nearly impossible, and I couldn't have access for a better one. Lastly, I'd like to thank Dr. Anderson. Little did I know what I'd be getting myself into that first day I walked into Dr. Anderson's class. I couldn't have imagined it would lead to 6 years of scholastic mentorship. I don't think I would have been able to accomplish half as much with any other professor. I know that I wouldn't be able to accomplish all the things I will accomplish without the knowledge and wisdom he has taught me. 
ACKNOWLEDGMENTS $\ldots \ldots \ldots \ldots \ldots \ldots$ ii

LIST OF TABLES $\ldots \ldots \ldots \ldots \ldots \ldots \ldots$ viii

LIST OF FIGURES $\ldots \ldots \ldots \ldots \ldots \ldots \ldots \ldots \ldots$

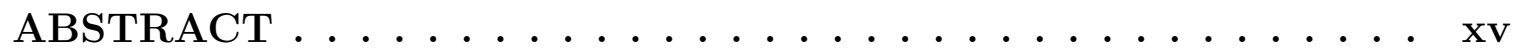

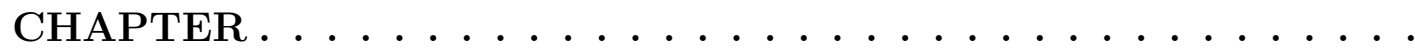

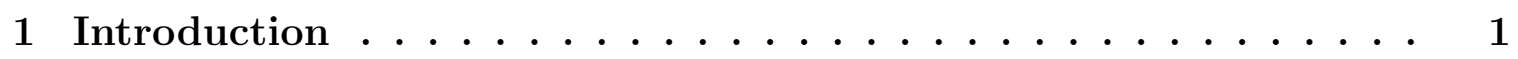

1.0 .1 State-of-the-Art in XAI $\ldots \ldots \ldots \ldots \ldots$

2 Explainable AI for Understanding Decisions and Data-Driven Optimization of the Choquet Integral $\ldots \ldots \ldots \ldots \ldots$

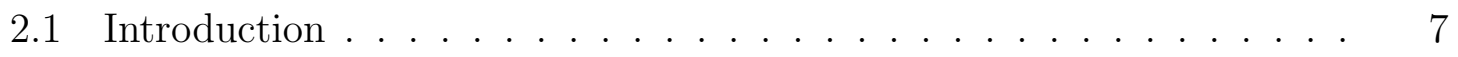

$2.2 \quad$ Measure and Choquet Integral $\ldots \ldots \ldots \ldots \ldots$

2.2 .1 Data-Driven Optimization of the Chl . . . . . . . . . 10

2.2 .2 Data Supported and Unsupported Variables . . . . . . . . . 11

$2.3 \quad$ Measure-Centric Indices: $f_{\mathrm{mc}}(\mu) \ldots \ldots \ldots \ldots \ldots$

$2.3 .1 \quad$ Shapley Index . . . . . . . . . . . . . . . . . . . . . . . . 13

2.3 .2 Interaction Index $\ldots \ldots \ldots \ldots \ldots$

$2.4 \quad$ Integral-Centric Indices: $f_{\text {ic }}\left(\mu, C_{\mu}\right) \ldots \ldots \ldots \ldots \ldots \ldots$

$2.5 \quad$ Data-Centric Indices: $f_{\mathrm{dc}}\left(\mu, C_{\mu}, O\right) \ldots \ldots \ldots \ldots \ldots$

2.5 .1 Variable Visitation . . . . . . . . . . . . . 16

2.5 .2 Percentage of Data Supported Variables $\ldots \ldots \ldots \ldots$

$2.5 .3 \quad$ Walk Visitation $\ldots \ldots \ldots \ldots \ldots$

2.5 .4 Percentage of LCSs Observed $\ldots \ldots \ldots \ldots \ldots$ 
2.5 .5 Dominant Walk Identification . . . . . . . . . . . . . 18

2.5 .6 Should we Trust our Fused Result? . . . . . . . . . . . . . . 18

$2.6 \quad$ Experiments and Results $\ldots \ldots \ldots \ldots$

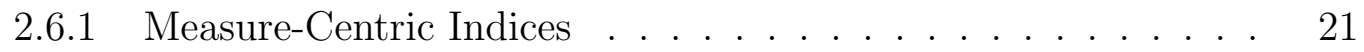

2.6 .2 Integral-Centric Indices $\ldots \ldots \ldots \ldots$

2.6 .3 Data-Centric Indices $\ldots \ldots \ldots \ldots \ldots$

$2.6 .4 \quad$ Measure + Integral + Data Index Combination . . . . . . 26

2.7 Conclusion and Future Work . . . . . . . . . . . . . . . . 26

3 Explainable AI for the Choquet Integral $\ldots \ldots \ldots \ldots$

$3.1 \quad$ Measure and Choquet Integral . . . . . . . . . . . . . . . . 30

3.1 .1 Optimization of the ChI $\ldots \ldots \ldots \ldots \ldots$

$3.1 .2 \quad$ Data Supported and Unsupported Variables . . . . . . . . . 33

$3.2 \quad$ Measure Centric Indices $\ldots \ldots \ldots \ldots$

$3.2 .1 \quad$ Shapley Index $\ldots \ldots \ldots \ldots \ldots$

$3.2 .2 \quad$ Walk Centric Shapley.$\ldots \ldots \ldots \ldots$

3.2 .3 Interaction Index $\ldots \ldots \ldots \ldots \ldots$

3.2 .4 Walk-Centric Interaction . . . . . . . . . . . . . . . . 37

3.3 Data-Centric Indices $\ldots \ldots \ldots \ldots$

3.3 .1 Variable Visitation . . . . . . . . . . . . . . . . . . . . 39

3.3 .2 Percentage of Data Supported Variables _. . . . . . . . . 39

3.3 .3 Walk Visitation . . . . . . . . . . . . . . . . . . . . . 40

3.3 .4 Percentage of Walks Observed . . . . . . . . . . . . . . . 41

3.3 .5 Dominant Walk Identification . . . . . . . . . . . . . . . . 41

$3.3 .6 \quad$ Complexity Analysis $\ldots \ldots \ldots \ldots$. . . . . . . . . . . . 42

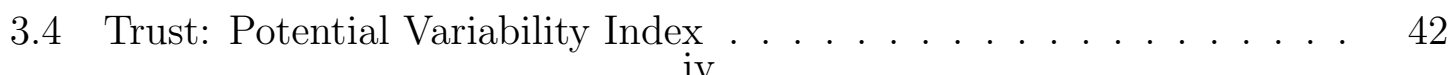


3.5 Experiments $\ldots \ldots \ldots \ldots \ldots$

$3.5 .1 \quad$ Shapley Index-Based Discoveries . . . . . . . . . . . . . . . 45

$3.5 .2 \quad$ Potential Variability Index-Based Discoveries . . . . . . . . . 45

3.5 .3 Data-Centric Index-Based Discoveries . . . . . . . . . . 46

3.5 .4 Overall XAI assessment $\ldots \ldots \ldots \ldots$. . . . . . . . . 47

3.6 Conclusion and Future Work . . . . . . . . . . . . . . . . . . . . . 49

4 Information Fusion-2-Text: Explainable Aggregation via Linguis-

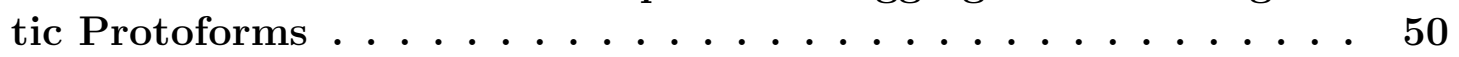

4.1 Introduction . . . . . . . . . . . . . . . . . . 50

$4.2 \quad$ Choquet Integral $\ldots \ldots \ldots \ldots \ldots \ldots a n$

$4.2 .1 \quad$ Optimization $\ldots \ldots \ldots \ldots \ldots \ldots \ldots$

$4.2 .2 \quad$ Data Supported Variables $\ldots \ldots \ldots \ldots$

4.3 Existing Low-Level XAI Indices _ . . . . . . . . . . . . . . . . 55

$4.3 .1 \quad$ Walk Centric Shapley . . . . . . . . . . . . . . . . . . 55

4.3 .2 Walk Visitation . . . . . . . . . . . . . . . . . . 56

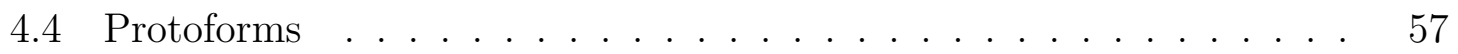

$4.5 \quad$ Fuzzy Sets $\ldots \ldots \ldots \ldots \ldots \ldots \ldots$

$4.5 .1 \quad$ Walk Centric Shapley . . . . . . . . . . . . . . . . . 58

4.5 .2 Walk Visitation . . . . . . . . . . . . . . . . . . . . . . . 59

4.6 Case Study $\ldots \ldots \ldots$

4.6 .1 Source Summaries . . . . . . . . . . . . . . . . . 60

4.6 .2 Code . . . . . . . . . . . . . . . . . . . . . 64

4.7 Summary and Future Work $\ldots \ldots \ldots \ldots \ldots$

5 Transfer Learning for the Choquet Integral . . . . . . . . . . . 66

5.1 Introduction $\ldots \ldots \ldots \ldots \ldots \ldots \ldots$ 
$5.2 \quad$ Fuzzy Measure and Integral $\ldots \ldots \ldots \ldots$

5.3 Data-Driven Learning.$\ldots \ldots \ldots \ldots$

5.4 Transfer Learning: Regularization-Based . . . . . . . . . . . . . 72

5.5 Transfer Learning: Two Step Optimization . . . . . . . . . . . . . 73

$5.6 \quad$ Experiments and Results $\ldots \ldots \ldots \ldots \ldots$

$5.6 .1 \quad$ Analytic Cases $\ldots \ldots \ldots \ldots \ldots \ldots$

Transfer t-conorm to t-norm . . . . . . . . . . . . . . . 76

Transfer t-norm to t-conorm . . . . . . . . . . . . . . 77

5.6 .2 Regularization versus Two Step Optimization $\ldots \ldots \ldots$. . . 78

5.7 Conclusions and Future Work $\ldots \ldots \ldots \ldots \ldots$

6 Actionable XAI for the Fuzzy Integral $\ldots \ldots \ldots \ldots$

6.1 Introduction $\ldots \ldots \ldots \ldots \ldots \ldots \ldots \ldots$

$6.2 \quad$ Fuzzy Measure and Integral $\ldots \ldots \ldots \ldots$

6.3 Existing XAI for the FI $\ldots \ldots \ldots \ldots \ldots \ldots$

6.3 .1 Information Indices $\ldots \ldots \ldots \ldots$

$6.3 .2 \quad$ Visual Explanations $\ldots \ldots \ldots \ldots$

$6.3 .3 \quad$ Local Explanations . . . . . . . . . . . . . . . . . . . . . 90

6.3 .4 Text or Linguistic Explanations . . . . . . . . . . . . . . . . 91

6.4 Actionable XAI and the FI . . . . . . . . . . . . . . . . . . 92

$6.4 .1 \quad$ Atomic Activity 1: Acquire New Data $\ldots \ldots \ldots \ldots . \ldots 92$

$6.4 .2 \quad$ Atomic Activity 2: Remove Source(s) _. . . . . . . . . . . 93

$6.4 .3 \quad$ Atomic Activity 3: Add Source . . . . . . . . . . . . . . 94

6.5 XAI Fusion Report . . . . . . . . . . . . . . . . . . . . . . . . . . . . 94

6.6 Case Studies . . . . . . . . . . . . . . . . . . . . . . . . . . . . 96

6.6 .1 Synthetic: Multi-Sensor Fusion . . . . . . . . . . . . 96 
$6.6 .2 \quad$ Real-World: Deep Learning for Remote Sensing . . . . . . . . 99

6.7 Conclusion and Future Work . . . . . . . . . . . . . . . . . . . . . . . 102

7 Conclusion . . . . . . . . . . . . . . . . 104

BIBLIOGRAPHY . . . . . . . . . . . . . . 108

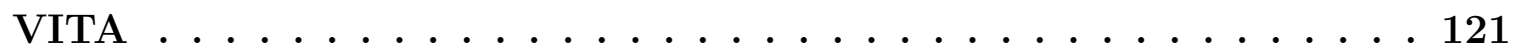




\section{LIST OF TABLES}

Table $\quad$ Page

2.1 UCM benchmark dataset . . . . . . . . . . . . . . . . . . 20

$2.2 \quad$ Accuracy of Individual NNs and their ChI Fusion $\ldots \ldots \ldots \ldots \ldots$

$3.1 \quad$ Shapley index for FM shown in Figure 2. . . . . . . . . . . . 35

$3.2 \quad$ Walk Centric Shapley for Figure $1 . \ldots \ldots \ldots \ldots$

3.3 Interaction index for Figure $2 . \ldots \ldots \ldots \ldots \ldots$

$3.4 \quad$ Walk-centric interaction index for Figure 2. . . . . . . . . . 38

$3.5 \quad$ Numerical example of the variable visitation and percentage of data supported variables indices. $\ldots \ldots \ldots \ldots$

$3.6 \quad$ Summary of the AID data set $\ldots \ldots \ldots \ldots \ldots$

$3.7 \quad$ Fusion Improvement on AID data set $\ldots \ldots \ldots \ldots \ldots \ldots$

$3.8 \quad$ Shapley index for AID data set: Shapley value (rank order value) . . 45

3.9 Data-centric indices for DCNN fusion on the AID data set . . . . . 48

4.1 Trapezoidal membership function parameters. . . . . . . . . . . . 59

$5.1 \quad$ Acronyms and Notation $\ldots \ldots \ldots \ldots \ldots \ldots$

5.2 Regularization Method: Many and Top-Down . . . . . . . . . . 80

5.3 Two Step Optimization: Many and Top-Down . . . . . . . . . . . . 81

5.4 Regularization Method: Many and Bottom-Up . . . . . . . . . . 81

5.5 Two Step Optimization: Many and Bottom-Up $\ldots \ldots \ldots \ldots$ viii 
6.1 Shapley values, percentage of walks observed, and accuracy of model when removing lowest valued source iteratively. . . . . . . . . . . . . 101 


\section{LIST OF FIGURES}

Figure

Page

1.1 Conventional data-driven life-cycle for implementing AI. $\ldots \ldots$. . . 2

1.2 DARPA reduced XAI to this set of questions. This figure also illustrates that XAI can occur in multiple places. . . . . . . . . . . . . . . 3

1.3 Common XAI terminology. . . . . . . . . . . . . . . . 3

1.4 XAI may come in different forms; this figure depicts three common forms, statistics, visualizations, or natural language. . . . . . . . . . . 4

$2.1 \quad$ Visualization of the FM and walk visitation frequency for the fusion of four deep convolutional neural networks for neuron one (agricultural) in the remote sensing UCM dataset. The most frequently encountered walk is $\left\{x_{1}\right\},\left\{x_{1}, x_{2}\right\},\left\{x_{1}, x_{2}, x_{3}\right\}$, then $\left\{x_{1}, x_{2}, x_{3}, x_{4}\right\}$ (which corresponds to the DCNNs CaffeeNet, GoogleNet, ResNet50 and ResNet101). 12

2.2 Visualization of the Shapley index values for (NN fold 1, fusion fold 1). Rows are the 21 classes and the $\mathrm{x}$-axis is the four NNs. Each row sums to one. . . . . . . . . . . . . . . . . . . . . . . . 22

$2.3 \quad$ Visualization of the interaction index for (NN fold 1, fusion fold 1) and classes 1 (upper left), 5 (upper right), 10 (lower left) and 15 (lower right). Values range from -1 to 1. . . . . . . . . . . . . . . . . . 22 
2.4 Visualization of the indices for introspection for (NN fold 1, fusion fold 1). Column one is $D_{1}(\max )$, two is $D_{2}(\min )$, three is $D_{3}$ (mean) and four is $D_{4}(\mathrm{LCOS})$.

2.5 Visualization of variable visitation frequency and percentage of data supported variables for (left) (NN fold 1, fusion fold 1) and (right) (NN fold 2, fusion fold 1). Variables appear according their binary encoded index. Row 1 are ideal values-meaning, if the variable visitations were uniformly distributed then that is the value (and thus color) they should be. Row 2 is NN class 1 , row 3 is NN class 2 , and so forth. Columns 1 to 15 are the FM variable according their binary encoded index. For example, column 1 is variable $\left\{x_{1}\right\}$, column 2 is $\left\{x_{2}\right\}$, column 3 is $\left\{x_{1}, x_{2}\right\}$, and so forth. Column 16 is the percentage of data supported variables for each class (relative to color and decimal point display resolution). . . . . . . . . . . . . . . . . . . . . . . . . . 24

2.6 (a) Percentage of LCS operators observed and dominant walk index. X-axis is output neuron (class) number. . . . . . . . . . . . . . . 25

3.1 Depiction of the XAI questions asked herein and their respective locations in the overall fusion process. . . . . . . . . . . . . . . . . . . . . 31

3.2 Visualization of an example FM for $\mathrm{N}=3$. All variables are observed except $\mu(x 1, x 3)$ (green). . . . . . . . . . . . . . . . . . . 35

$3.3 \quad$ Potential variability for classes, 6,8 , and 28 . . . . . . . . . . . . . . . 47 
4.1 Graphical illustration of fusion-2-text for the case study explored herein involving object detection and land cover classification in remote sensing. First, multiple machine learning (ML) models are trained in a cross validation context. Next, fusion is used to combat the fact that no single ML architecture is best across all data and classes. However, what have we learned? Fusion-2-text is used to discover a succinct set of important summaries or anomalies for a user in or across models. . $\quad 50$

4.2 Illustration of computational stages in our fusion-2-text. . . . . . . . 54

4.3 Vocabulary used herein to produce fusion LPSs. . . . . . . . . . . . . 58

4.4 Actual versus the ideal distribution of quantifier. . . . . . . . . . . . 61

4.5 LPSs describing the importance of each DCNN with truth degree (gray). 62

4.6 Most specific walks are observed. . . . . . . . . . . . . . . . . . 63

5.1 Example FM for the case of $N=4$. Circles denote subsets and edges denote monotonic inequalities. . . . . . . . . . . . . . . . . . 69

5.2 Example FM where a few variables are missing (red). . . . . . . . . . 76

$5.3 \quad$ Example FM where many variables are missing (red). . . . . . . . . . 77

5.4 Transfer learning where $\mu^{S}$ is the maximum and $\mu^{T}$ is the minimum. The variable in red is missing towards the top of the lattice and its transfer value is zero. . . . . . . . . . . . . . . . . . . . . . . . . . . . 78

5.5 Transfer learning where $\mu^{S}$ is the maximum and $\mu^{T}$ is the minimum. The variable in red is missing towards the bottom of the lattice and its transfer value is one. . . . . . . . . . . . . . . . . . . . . . . . . 79

5.6 Transfer learning where $\mu^{S}$ is the minimum and $\mu^{T}$ is the maximum. The variable in red is missing towards the top of the lattice and its transfer value is one. . . . . . . . . . . . . . . . . . . . . . . 80 
5.7 Transfer learning where $\mu^{S}$ is the minimum and $\mu^{T}$ is the maximum. The variable in red is missing towards the bottom of the lattice and its transfer value is zero. . . . . . . . . . . . . . . . . . . . . . . . . . 82

5.8 (Regularization Method) Error (y-axis) associated with transfer learn-

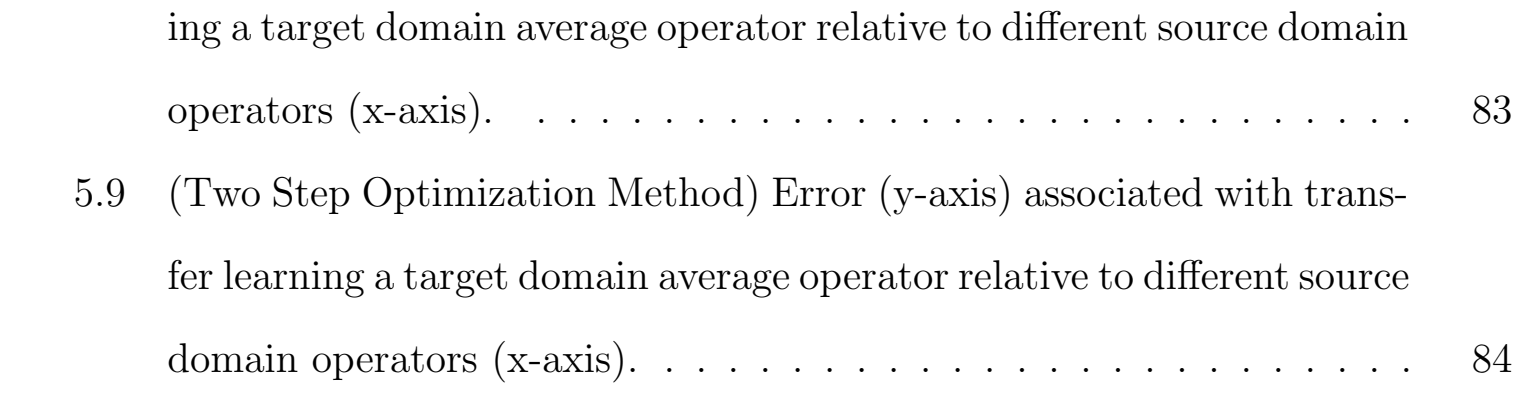

6.1 Rows are learned ChIs (one per class in the AID dataset), row one is the ideal case, and color coding goes from blue (value 0) through white (0.5) to red (1). (left) Columns are Shapley values. (right) Columns are FM variable observation frequency (lexicographical coding) and the last row is the total number of observed walks. . . . . . . . . . . . . . 89

6.2 (left) Example Hasse diagram for a ChI whose training data observed

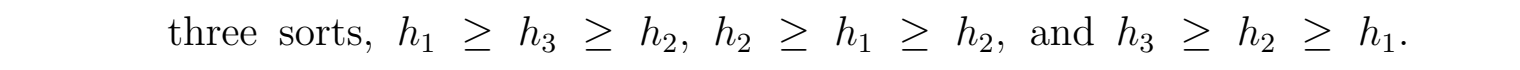
The bottom is $\mu(\emptyset)$, the next layer up is $\left\{\mu_{1}, \mu_{2}, \mu_{3}\right\}$, layer two is $\left\{\mu_{1,2}, \mu_{1,3}, \mu_{2,3}\right\}$, and the top is $\mu(X)$. (right) Real diagram for the benchmark Yacht data and non-monotonic ChI for regression [98]. . . 90

6.3 Subset of the vocabulary for generating linguistic protoform summaries for XAI for the ChI. Introduced in $[99]$. . . . . . . . . . . . . . . . . 91

6.4 Actionable XAI fusion report template. The left column has context, actions, and assistance. The right column has low-level XAI details, e.g., linguistic, graphical, etc. Each box has has a link to expand if a user desires access to the full set of underlying explanations. . . . . . 95 
6.5 Case Study 1: Synthetic two dimensional dataset with 3 sensors (sources), a ground truth, and fusion results. Red color coded samples have a value of 0 and blue indicates 1 . The answer is the minimum of sources 1 and $3 . \ldots \ldots \ldots . \ldots \ldots 6$

6.6 Case Study 1: Example fusion report recommending add a source. This action is supported by a small set of rank ordered low-level XAI explanations $($ right column). . . . . . . . . . . . . . . . . . . . . . . . 98

6.7 Case Study 1: Report showing that a source can be removed and no irregularities or anomalies otherwise exist. . . . . . . . . . . . . . . . 98

6.8 Case Study 2: The recommended actions are to remove sources and acquire more data. The visual explanations show that the model was supported by an extremely low amount of training data and there are many Shapley values indicating that sources could likely be removed. 100

6.9 Case Study 2: More in-depth interface showing a rank ordering-by ascending probability-on walks (context). Context with little-to-no probability of observation require attention and additional data collection. . . . . . . . . . . . . . . . . . . . 101 


\begin{abstract}
This is the abstract of your dissertation project. It should not exceed one page.

The recent resurgence of Artificial Intelligence (AI), specifically in the context of applications like healthcare, security and defense, IoT, and other areas that have a big impact on human life, has led to a demand for eXplainable AI (XAI). The production of explanations is argued to be a key aspect of achieving goals like trustworthiness and transparent versus opaque AI. XAI is also of fundamental academic interest with respect to helping us identifying weaknesses in the pursuit of making better AI. Herein, I focus on one piece of the AI puzzle, information fusion. In this work, I propose XAI fusion indices, linguistic summaries (aka textual explanations) of these indices, and local explanations for the fuzzy integral. However, a limitation of these indices is its tailored to highly educated fusion experts, and it is not clear what to do with these explanations. Herein, I extend the introduced indices to actionable explanations, which are demonstrated in the context of two case studies; multi-source fusion and deep learning for remote sensing. This work ultimately shows what XAI for fusion is and how to create actionable insights.
\end{abstract}




\section{Chapter 1}

\section{INTRODUCTION}

Artificial intelligence (AI) is moving full steam ahead, regardless of our understanding of it. The majority of modern AI, which is arguably more machine learning (ML) than AI, consists of data-driven task specific algorithms. Each incremental step forward in the research community contributes to the big AI picture; from better data collection practices to mathematics, hardware, and everything in between. In the last decade, AI has left the hands of researchers and it is now being used in real-world scenarios. For example, many automotive companies are investing billions of dollars to develop and implement AI into autonomous vehicles [1]. In the medical industry, AI has found use cases in detecting disease and even transcribing a physician's notes [2]. In Agriculture, AI is being used for tasks from precision agriculture to food security [3]. Some applications even require that the AI make life-or-death decisions. Specifically, autonomous cars impact the safety of those in and around vehicles, medical AI is important when it impacts diagnoses, and automatic target recognition is used for security and defense by governments. For those familiar with the mechanics of AI, this can be a frightening reality. In fact, it has prompted countries and organizations around the world to implement AI guidelines [4]. Even the White House has written "AI with American Values" to start a foundation of how to protect the American people. Fortunately, there is a thrust for AI systems that are explainable.

It is common for AI approaches to follow similar workflows-specifically ap- 
proaches driven by data. This life-cycle is generalized in Figure 1.1. However, when implementing AI, it is often not analytically clear as to what methods are best. Modern $\mathrm{AI}$ is a process that requires pivoting when an approach may not be working and understanding what parameters to tune to realize the desired solution. The process of tuning is complete once all of the problem's criteria are met.

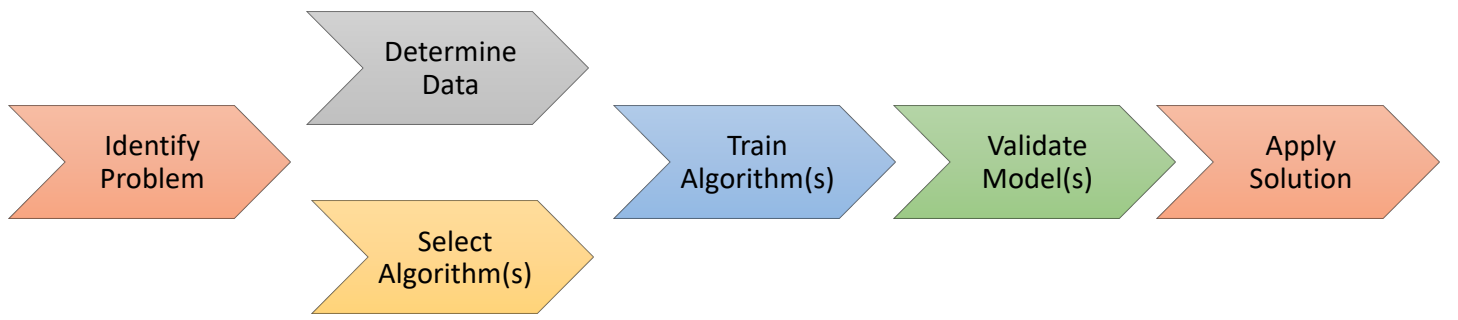

Figure 1.1: Conventional data-driven life-cycle for implementing AI.

This summary of data-driven AI was provided to highlight that there are multiple points of failure. Since modern AI is as much art as science, how do we trust an AI solution? Any arrow in Figure 1.1 is a vulnerability. One common issue is that these vulnerabilities lead to bias. The authors of [5] present the different ways in which bias has surfaced in applications including (but not limited to) the judicial system, beauty pageants, and facial recognition software. Explainable AI (XAI) is a new field that seeks to mitigate unwanted vulnerabilities and help us build trust in an AI solution. This begs the question of what is XAI? Unfortunately, there is no consensus on what constitutes XAI, and defining seems to be an ever moving target (much like AI). As shown in Figure 1.2, organizations like DARPA [6] have raised questions to get at the heart of XAI by focusing on the relationship of how a model is built by an algorithm in terms of data, and explanations can occur at any point in this process.

The research community has begun to identify terminology and in some cases an ontology for XAI (see 1.3). However, these definitions can often be confusing and many times terms are defined in terms of one another. 


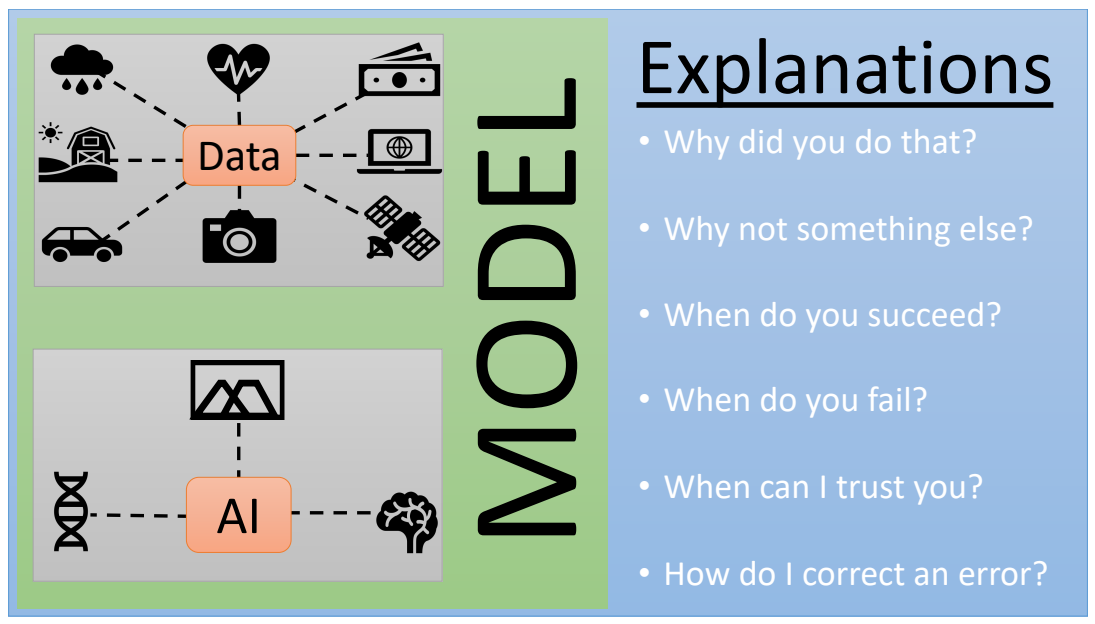

Figure 1.2: DARPA reduced XAI to this set of questions. This figure also illustrates that XAI can occur in multiple places.

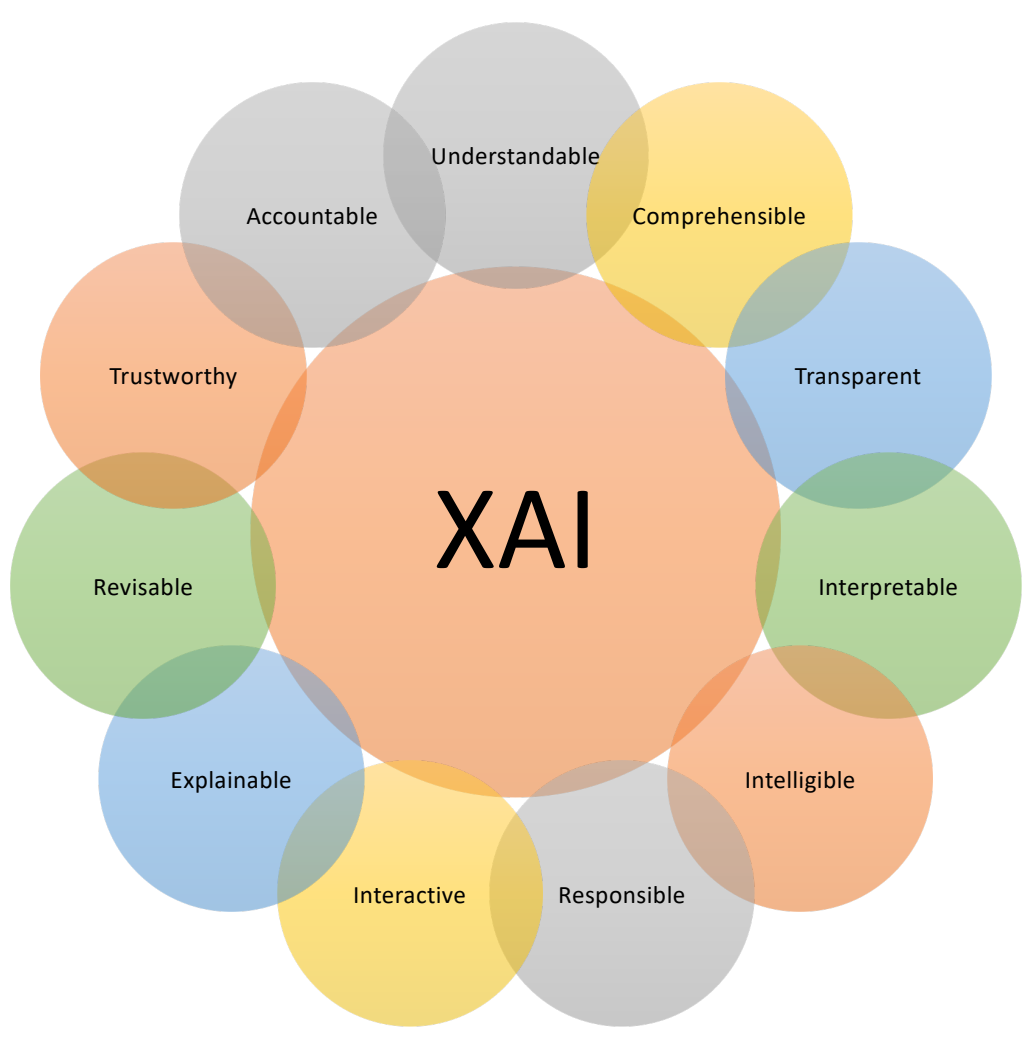

Figure 1.3: Common XAI terminology. 
Beyond the question of how do we define XAI, there is the question of how should information be delivered to a user (see Figure 1.4)? In many cases an effective explanation can take the form of statistical analysis. In other scenarios it is better to provide visualizations or even linguistic descriptions. While this list of ways to facilitate human-machine interaction is not exhaustive, it does shed light on the prevailing issue of not only what is an explanation, but how do we transfer this knowledge to a human. In part, what makes this problem so hard is the reality that optimal explanations are likely application specific and even perhaps context dependent.

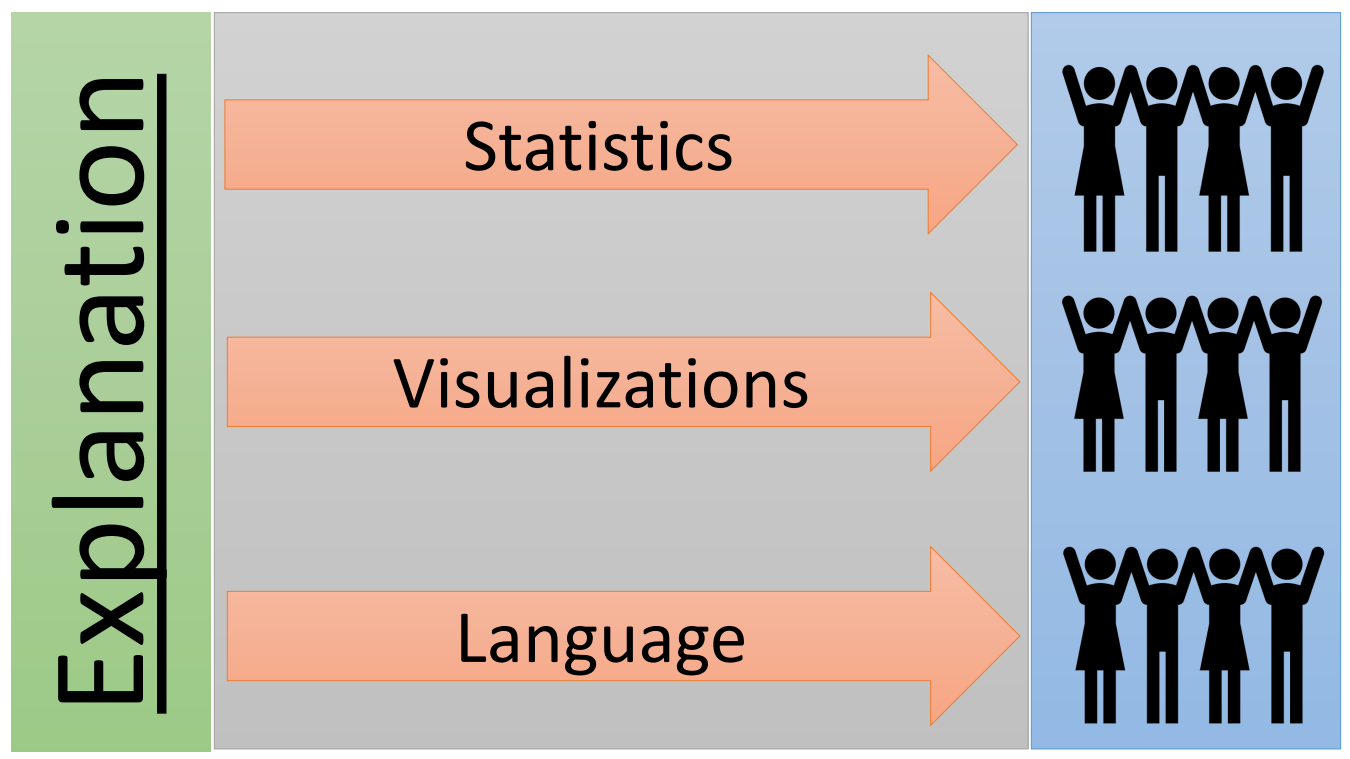

Figure 1.4: XAI may come in different forms; this figure depicts three common forms, statistics, visualizations, or natural language.

While the above summarizes the ever evolving XAI landscape, this thesis identifies and investigates the following specific challenges related to XAI for information fusion. 
1. How are low-level explanations extracted from data for fusion?

2. How are low-level explanations extracted from models for fusion?

3. How are high-level visual and linguistic fusion explanations extracted?

4. How can this knowledge be used to transfer fusion solutions across domains?

\subsubsection{State-of-the-Art in XAI}

As stated above, there are many algorithms and approaches to AI. The following is a brief summary of current trends in XAI. At a high-level, XAI can generally be divided into XAI that extracts information a model (often a black box) or XAI by design. For example, deep learning has led to astonishing leaps in performance, but generally at the cost of opaqueness. Most XAI to date has focused on understanding deep learners based on a learned model. For instance, in [7, 8, 9, 10], researchers produced visual explanations to understand convolutional neural networks, usually on a premise of back flowing visual attention relative to a feature or machine decision (e.g., class label). On the other hand, in LIME [11], Ribeiro et al. explored XAI methods that study the relationship between inputs and outputs by perturbing the input to see how it affects the output. In [12], Lundberg exploited a game-theoretic approach of Shapley values to understand the importance of different components in an AI. Moreover, even businesses such as LinkedIn have produced XAI tools to determine bias in an AI [13].

If black box XAI is on one end of the spectrum, explainability by design is on the other. One community that on occasion claims explainability by design is the fuzzy logic community. Fuzzy logic is built on the notion of rules, and the argument is sometimes that rules are more interpretable than deep learning. In theory, the learned rules, themselves, are explainable. This property has led to the learning of fuzzy rules. In [14], Sanz et al. learned a fuzzy rule based classifier that produces rules 
to classify financial data. Furthermore, in [15], Hagras argues that type-2 fuzzy logic provides very effective XAI because this math mimics the way that humans think. Granted, one of the lingering questions deals with the trade-off between performance and explainability; meaning, attempting to understand how much performance is sacrificed for explainability. The authors of [16] address this question with respect to two methods of learning a fuzzy logic system-ANFIS 17] and ADONiS [18. The point is, research exists in some communities to address the design of improved representations for understandability. However, there are questions if that is true when these tools are intersected with our current data driven era.

While the above is a terse summary of past and recent work (see [19] and 20] for a recent review of XAI), the focus of this thesis is data/information fusion, a topic not necessarily covered by the above approaches. In particular, this is important because there are unique questions related to the syntax and the semantics of XAI in fusion versus what others are exploring. The following thesis is broken up into four parts. Chapter 2 details a way to extract explanations from data and models relative to fusion. Chapter 3 considers the question of how to elicit meaningful explanations from incomplete models; which exists by definition on data driven domains. Chapter 4 takes this work and considers how to report it to a human linguistically. Last, Chapter 5 explores if these methods can be leveraged to intelligently transfer a solution from one domain (called the source) to a new domain (called the target). Overall, these chapters detail how to elicit meaningful explanations, how to communicate this information to people, and applications. 


\section{Chapter 2}

\section{EXPLAINABLE AI FOR UNDERSTANDING DECISIONS AND DATA-DRIVEN OPTIMIZATION OF THE CHOQUET INTEGRAL}

\subsection{INTRODUCTION}

Data and information fusion is a fundamental capability in many state-of-the-art technologies, e.g., Big Data, smart cars, remote sensing, computer vision, etc. However, fusion is a rather vague concept that takes many forms. For example, in remote sensing sensor registration is fusion. In computer vision, the combining of features is fusion. In expert systems, the combining of expert opinions is fusion. Herein, we restrict our analysis and focus on a more specific avenue of fusion: aggregation functions. Let $X=\left\{x_{1}, \ldots, x_{N}\right\}$ be $N$ sources like a sensor, human or algorithm. In general, an aggregation function is a mapping of data from our $N$ sources, denoted by $h\left(x_{i}\right) \in \Re$, to data, $f\left(\left\{h\left(x_{1}\right), \ldots, h\left(x_{N}\right)\right\}, \Theta\right) \in \Re$, where $\Theta$ are the parameters of f. Fusion typically needs a "home" to make sense, e.g., fusion for machine learning, fusion for scientific visualization, etc. The point is, in order to evaluate the success of fusion one typically needs a context in which to explore the quality of its result. Common nomenclature for fusion of different data is signal-in-signal-out (SISO), feature-in-feature-out (FIFO) and decision-in-decision-out (DIDO). Herein, we focus on the Choquet integral (ChI), which has been used for SISO, FIFO and DIDO. 
Often, it is too complicated or not possible for a human to specify the parameters of the underlying aggregation operator. In response, a multitude of methods have appeared on data-driven optimization of aggregation operators, e.g., 21]. The subject of this article is, learning a solution from data is useful, but what is the quality of that learned solution and why should we trust decisions that it produces on new data? In part, the current article is driven by the emerging need for so-called explainable artificial intelligence (XAI). Herein, we explore XAI methods for introspection of the ChI. As such, we pull together existing methods and explore new data-driven ones.

In Section 2.2 we review the ChI, Section 2.3 is existing fuzzy measure (FM) specific XAI tools, Section 2.4 is existing ChI specific XAI methods, and Section 2.5 is new data-centric XAI methods. Last, in Section 2.6 we use these XAI methods to fuse a heterogeneous set of deep convolutional neural networks (DCNN) for remote sensing on benchmark data sets.

\subsection{MEASURE AND CHOQUET INTEGRAL}

The fuzzy integral has been demonstrated numerous times in a variety of applications; e.g., explosive hazard detection 22, 23], computer vision [24], pattern recognition 25, 26, 27], multi-criteria decision making [28, 29], forensic anthropology [30, 31], fuzzy logic [32], multiple kernel learning [33], multiple instance learning [34], ontologies 35], missing data [36, and deep learning for remote sensing 37, 38], to name a few. The $\mathrm{ChI}$ is a nonlinear aggregation function parameterized by the FM. Herein, we focus on the real-valued discrete (finite $X$ ) ChI.

The first action we face is how to assign "worth" to different subsets of sources. For example, the well-known backbone of calculus on real-valued domains is the Lebesgue measure; which coincides with length, area and hypervolume. However, when $X$ is a discrete domain, e.g., set of algorithms, what is the corresponding measure? In [24], Keller et al. first investigated the idea of using the fuzzy integral for pattern 
recognition. A FM is a function, $\mu$, on the power set of $X, 2^{X}$, which satisfies (1) (boundary condition) $\mu(\emptyset)=0$ and (2) (monotonicity) if $A, B \subseteq X$ and $A \subseteq B$, then $\mu(A) \leq \mu(B)$. Often, $\mu(X)=1$ is imposed in settings like DIDO fusion.

The FM models interactions (e.g., subjective worth, statistical correlation, etc.) between input subsets. The data provided by our inputs are $\left\{h\left(\left\{x_{1}\right\}\right), h\left(\left\{x_{2}\right\}\right), \ldots, h\left(\left\{x_{N}\right\}\right)\right\}$. The fuzzy integral is a way to combine the integrand $(h)$ data relative to the FM $(\mu)$. Let $h\left(\left\{x_{i}\right\}\right) \in \Re^{\geq 0}$ be the data/information from input $i$. The discrete (finite $X$ ) Sugeno FI is 1

$$
\int_{S} h \circ \mu=S_{\mu}(h)=\bigvee_{i=1}^{N}\left(h\left(\left\{x_{\pi(i)}\right\}\right) \wedge \mu\left(A_{i}\right)\right),
$$

where $\pi$ is $h\left(\left\{x_{\pi(1)}\right\}\right) \geq h\left(\left\{x_{\pi(2)}\right\}\right), \ldots, \geq h\left(\left\{x_{\pi(N)}\right\}\right)$ and $A_{i}=\left\{x_{\pi(1)}, \ldots, x_{\pi(i)}\right\}$. The discrete ChI is ${ }^{2}{ }^{3}$

$$
\int_{C} h \circ \mu=C_{\mu}(h)=\sum_{i=1}^{N} h\left(\left\{x_{\pi(i)}\right\}\right)\left[\mu\left(A_{i}\right)-\mu\left(A_{i-1}\right)\right],
$$

where $\mu\left(A_{0}\right)=0$. Since the ChI is a parametric aggregation function, once the FM is determined the ChI turns into a specific operator. For example: if $\mu(A)=1, \forall A \in$ $2^{X} \backslash \emptyset$, the ChI becomes the maximum operator; if $\mu(A)=0, \forall A \in 2^{X} \backslash X$, we recover the minimum; if $\mu(A)=\frac{|A|}{N}$, we recover the mean; and for $\mu(A)=\mu(B)$ when $|A|=|B|, \forall A, B \subseteq X$, we obtain a linear combination of order statistics (LCOS). In general, each of these cases can be viewed as constraints or simplifications on the FM (and therefore the ChI).

The discrete ChI can alternatively be regarded as $N$ ! linear convex sum (LCS)

\footnotetext{
${ }^{1}$ Due to the maximum (t-conorm) and minimum (t-norm) operators, the Sugeno FI does not actually generate any possible number between the minimum and maximum of the inputs. Instead, it selects one of the FM or input values, i.e., at most one of $2^{N}+N$ values.

${ }^{2}$ The $\mathrm{ChI}$ is used frequently for a number of reasons; e.g., it is differentiable 26, for an additive (probability) measure it recovers the Lebesgue integral, it yields a wider (versus the Sugeno integral) spectrum of values, etc.

${ }^{3}$ If $\mu(X)<1$, idempotence, boundedness, etc. are not guaranteed.
} 
operators-one for each possible input sort $4^{4}$ For a single instance of the ChI, i.e., $N$ new inputs that need to be fused, its sort determines which LCS to use. If we consider a constrained FM then this set of LCSs is reduced. For example, a LCOS has one underlying operator. Each sort has a corresponding "walk" in the FM. For example, let $N=3$ and $h\left(\left\{x_{2}\right\}\right) \geq h\left(\left\{x_{3}\right\}\right) \geq h\left(\left\{x_{1}\right\}\right)$. Equation 2.2 thus uses the FM variables $\mu(\emptyset), \mu\left(\left\{x_{2}\right\}\right), \mu\left(\left\{x_{2}, x_{3}\right\}\right)$ and $\mu(X)$. This sequence of increasing cardinality FM variables is referred to as a "walk" up the FM lattice (Hasse diagram) hereafter 5

\subsubsection{Data-Driven Optimization of the ChI}

A big question is, where do we get the FM from? One option is to have an expert specify it. However, this is not practical (assuming the expert could even meaningfully assign values to the interactions) as the number of inputs increases. Another option is we can specify or learn the worth of just the singletons (the densities). From there, a number of formulas can be used to impute (fill in) the missing variable values. Popular approaches include the Sugeno $\lambda$-FM and the S-Decomposable FM [39]. However, while convenient, most often we do not obtain the desired values for variables that we need. Next, we quickly review one method of optimizing the ChI. The reader can refer to 21] for more details (full mathematical detail and experiments).

Let $O=\left\{\mathbf{h}_{j}, y_{j}\right\}, j=1, \ldots, M$, be $M$ training examples; where $\mathbf{h}_{j}$ is the $j$-th instance with data/information from $N$ inputs and $y_{j}$ is the ground-truth for $\mathbf{h}_{j}$. The sum of squared error for training dataset $O$ is

$$
E(O, \mathbf{u})=\sum_{j=1}^{M} e^{j}=\sum_{j=1}^{M}\left(\mathbf{c}_{j}^{T} \mathbf{u}-y_{j}\right)^{2}=\|D \mathbf{u}-\mathbf{y}\|_{2}^{2}
$$

\footnotetext{
${ }^{4}$ Whereas there are $N$ ! LCS operators, they share $2^{N}$ variables. For example, for $N=6$ we have 720 LCS operators but only 64 variables.

${ }^{5}$ As $\mu(\emptyset)=0$ and $\mu(X)=1$, it is trivial to prove that the difference-in-measure coefficients in Equation 2.2 sum to 1.
} 
where $\mathbf{u}=\left[\mu\left(\left\{x_{1}\right\}\right), \ldots, \mu\left(\left\{x_{1}, x_{2}\right\}\right), \mu\left(\left\{x_{1}, x_{3}\right\}\right), \ldots, \mu(X)\right]$ (lexiographic vector of size $\left.2^{N}-1\right), D=\left[\begin{array}{llll}\mathbf{c}_{1} & \mathbf{c}_{2} & \ldots & \mathbf{c}_{M}\end{array}\right]^{T}$ (full dataset), $\mathbf{y}=\left[\begin{array}{llll}y_{1} & y_{2} & \ldots & y_{M}\end{array}\right]^{T},\|\cdot\|_{2}$ is norm-2 operation, and $\mathbf{c}_{j}$ holds the coefficients of $\mathbf{u}$ for observation $\mathbf{h}_{j}$, e.g., for $N=3$ and $h\left(\left\{x_{2}\right\}\right) \geq h\left(\left\{x_{1}\right\}\right) \geq h\left(\left\{x_{3}\right\}\right), c$ is

$$
\left[0, h\left(\left\{x_{2}\right\}\right)-h\left(\left\{x_{1}\right\}\right), 0, h\left(\left\{x_{1}\right\}\right)-h\left(\left\{x_{3}\right\}\right), 0,0, h\left(\left\{x_{3}\right\}\right)\right]
$$

The regularized SSE optimization problem is

$$
\min _{\mathbf{u}} f(\mathbf{u})=\|D \mathbf{u}-\mathbf{y}\|^{2}+\beta v(\mathbf{u})
$$

where $\beta \in \Re^{\geq 0}$ is a regularization constant (which balances the "cost" (or penalty) of obtaining minimum function error relative to our desire to have minimal model complexity) and $v(\mathbf{u})$ is an index of model complexity (e.g., k-additive and Mobius, Gini-Simpson, $\ell_{p}$-norm, etc. [40]), subject to the FM boundary and monotonicity conditions (see [41] for how to pack the constraints into a linear algebra expression), which can be solved via quadratic programming. Full code and explanation (including how to build the constraint matrix $C$ ) can be found at www.derektanderson.com/FuzzyLibrary.

\subsubsection{Data Supported and Unsupported Variables}

Many parametric methods are unable to determine which variables are supported (and to what degree at that) by data. For example, determination of which parameters are sufficiently approximated by data in a neural network is unsolved. In [21], we put forth a way to identify data supported and data unsupported FM variables for learning the FM/ChI from data. For $M$ training instances we get, at maximum, $M$ unique sorts 6 . For each walk, we record which variables are used. Data supported

\footnotetext{
${ }^{6}$ In practice, it is not common that we encounter $M$ unique sorts for $M$ instances. Depending on the diversity of our data, it is more common to have just a small percentage of unique walks. The number of walks typically becomes sparser as $N$ increases.
} 


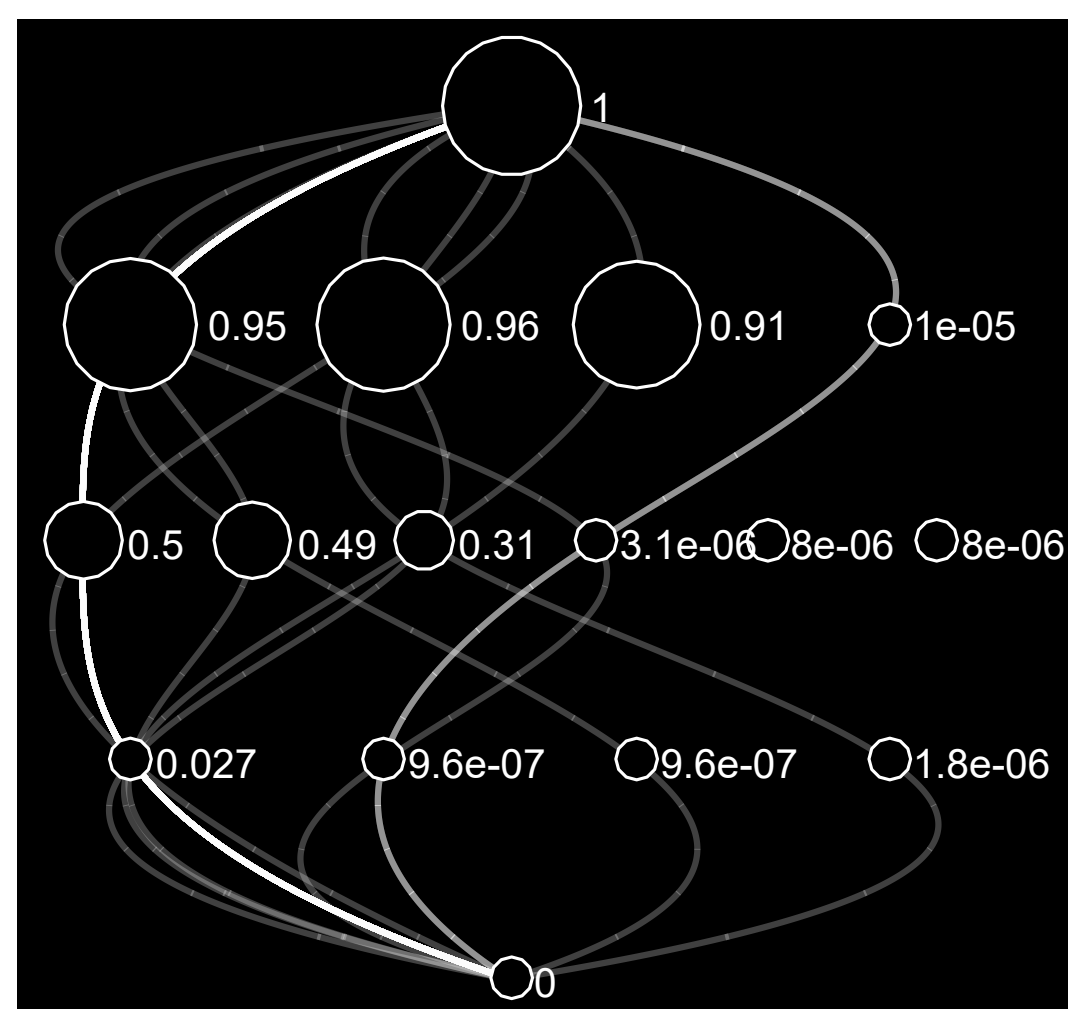

Figure 2.1: Visualization of the FM and walk visitation frequency for the fusion of four deep convolutional neural networks for neuron one (agricultural) in the remote sensing UCM dataset. The most frequently encountered walk is $\left\{x_{1}\right\}$, $\left\{x_{1}, x_{2}\right\},\left\{x_{1}, x_{2}, x_{3}\right\}$, then $\left\{x_{1}, x_{2}, x_{3}, x_{4}\right\}$ (which corresponds to the DCNNs CaffeeNet, GoogleNet, ResNet50 and ResNet101).

means a FM variable $(A \subseteq X)$ was encountered at least once. Data unsupported means a variable was never encountered in the $M$ walks.

Figure 2.1 shows the $2^{N} \mathrm{FM}$ variables for an $N=4$ problem-specifically the fusion of DCNNs. "Layer" $L$ (from bottom to top) in the image denotes FM variables with cardinally $L$. Thus, layer 0 (bottom node) is the empty set, the next layer is the singletons (from left to right), top is $\mu(X)$, etc. Each variable is presented in lexicographic order, i.e., layer 2 is $\left\{x_{1}, x_{2}\right\},\left\{x_{1}, x_{3}\right\},\left\{x_{1}, x_{4}\right\},\left\{x_{2}, x_{3}\right\},\left\{x_{2}, x_{4}\right\}$ and $\left\{x_{3}, x_{4}\right\}$. The nodes are also drawn size-wise proportional to their value (a minimum size and maximum was specified to make them still show up for 0-valued variables). In addition, the "paths" drawn indicate the visitation frequency (the brighter the line, the higher the visitation) for test data in fold 1. 


\subsection{MEASURE-CENTRIC INDICES: $F_{\mathrm{MC}}(\mu)$}

Next, we review the first of three approaches to understand the inner-workings of fusion. In general, we have $\left(\mu, C_{\mu}, O\right)$ at our disposal-the measure, integral and data set respectively. This section focuses on questions that require just $\mu$.

\subsubsection{Shapley Index}

The Shapley index informs us about the "worth" of each input,

$$
\begin{aligned}
\Phi_{\mu}(i) & =\sum_{K \subseteq X \backslash\{i\}} \zeta_{X, 1}(K)(\mu(K \cup\{i\})-\mu(K)), \\
\zeta_{X, 1}(K) & =\frac{(|X|-|K|-1) !|K| !}{|X| !}
\end{aligned}
$$

where $K \subseteq X \backslash\{i\}$ denotes all proper subsets from $X$ that do not include source $i$. The Shapley value of $\mu$ is the vector $\Phi_{\mu}=\left(\Phi_{\mu}(1), \ldots, \Phi_{\mu}(N)\right)^{t}$ and $\sum_{i=1}^{N} \Phi_{\mu}(i)=1$. The Shapley index can be interpreted as the average amount of contribution of source $i$ across all coalitions.

\subsubsection{Interaction Index}

The Interaction Index informs us about how two inputs interact with one anotheraka what advantage (or not) is there in combining inputs. The Interaction Index (Murofushi and Soneda [42]) between $i$ and $j$ is

$$
\begin{aligned}
\mathcal{I}_{\mu}(i, j) & =\sum_{K \subseteq X \backslash\{i, j\}} \zeta_{X, 2}(K)(\mu(K \cup\{i, j\}) \\
& -\mu(K \cup\{i\})-\mu(K \cup\{j\})+\mu(K)), \\
\zeta_{X, 2}(K) & =\frac{(|X|-|K|-2) !|K| !}{(|X|-1) !}
\end{aligned}
$$


where $\mathcal{I}_{\mu}(i, j) \in[-1,1], \forall i, j \in\{1,2, \ldots, N\}$. A value of 1 (respectively, -1 ) represents the maximum complementary (respective redundancy) between $i$ and $j$. The reader can refer to [43] for further details about the interaction index, its connections to game theory and interpretations. Grabisch extended the index to the general case of any coalition 44 ,

$$
\begin{aligned}
\mathcal{I}_{\mu}(A) & =\sum_{K \subseteq X \backslash A} \zeta_{X, 3}(K, A) \sum_{C \subseteq A}(-1)^{|A \backslash C|} \mu(C \cup K), \\
\zeta_{X, 3}(K, A) & =\frac{(|X|-|K|-|A|) !|K| !}{(|X|-|A|+1) !}
\end{aligned}
$$

Equation (2.7) is a generalization of both the Shapley index and Murofushi and Soneda's interaction index as $\Phi_{\mu}(i)$ corresponds with $\mathcal{I}_{\mu}(\{i\})$ and $\mathcal{I}_{\mu}(i, j)$ with $\mathcal{I}_{\mu}(\{i, j\})$.

\subsection{INTEGRAL-CENTRIC INDICES: $F_{\text {IC }}\left(\mu, C_{\mu}\right)$}

A different fundamental question is what "type" of aggregation is the ChI performing? Answering this question helps us understand how the inputs are being combined (e.g., in an optimistic, pessimistic, expected value like fashion, etc.). In this section we review indices that operate on $\left(\mu, C_{\mu}\right)$.

In [45], we established an index $D_{1}$ to measure the degree to which a given $\mathrm{FM} / \mathrm{ChI}$ is an maximum operator. Let "layer k" (measure defined on sets of cardinality $k$ ) be denoted by $L(k)$, e.g., $L(1)=\left\{\mu\left(\left\{x_{1}\right\}\right), \mu\left(\left\{x_{2}\right\}\right), \mu\left(\left\{x_{3}\right\}\right)\right\}$ for $N=3$.

$$
\begin{gathered}
D_{1}(\mu)=\sum_{k=1}^{1} \frac{\mathbf{W}(k)}{2}\left(T_{1}+T_{4}\right)+\left[\sum_{k=2}^{N} \frac{\mathbf{W}(k)}{3}\left(T_{1}+T_{2}+T_{4}\right)\right] \\
T_{1}=1-\left(\frac{\sum_{I \in L(k)} \mu(I)}{|L(k)|}\right), \\
T_{2}=\left(\frac{\sum_{I \in L(i)} \mu(I)}{|L(k)|}-\frac{\sum_{J \in L(k-1)} \mu(J)}{|L(k-1)|}\right)
\end{gathered}
$$




$$
\begin{gathered}
T_{3}=\frac{\sum_{I \in L(k)} \mu(I)}{|L(k)|}, \\
T_{4}=\frac{\sum_{I \in L(k)}\left(\mu(I)-T_{3}\right)^{2}}{|L(k)|-1}, \\
\mathbf{W}=\frac{\left[\frac{1}{N}, \ldots, 1\right]}{\sum_{i=1}^{N} \frac{i}{N}} .
\end{gathered}
$$

In summary, $T_{4}$ is the variance of a layer, $T_{3}$ is the mean of a layer, $T_{2}$ is the difference in mean value between two consecutive layers, $T_{1}$ is how far the mean of a layer is from value 1 and $\mathbf{W}$ is a set of layer weights. A value of $D_{1}=0$ means that a $\mathrm{ChI}$ is the maximum operator. In addition, the distance of a learned capacity to a minimum operator $\left(D_{2}\right)$, mean $\left(D_{3}\right)$ and $\operatorname{LCOS}\left(D_{4}\right)$ is

$$
\begin{gathered}
D_{2}(\mu)=\sum_{k=1}^{1} \frac{\mathbf{W}_{2}(k)}{2}\left(T_{3}+T_{4}\right) \\
+\left[\sum_{k=2}^{N-1} \frac{\mathbf{W}_{2}(i)}{3}\left(T_{3}+T_{2}+T_{4}\right)\right], \\
D_{3}(\mu)=\frac{1}{2^{N}-2} \sum_{k=1}^{N-1} \sum_{I \in L(k)}\left|\mu(I)-\frac{k}{N}\right|, \\
D_{4}(\mu)=\frac{1}{N-1} \sum_{k=1}^{N-1} \sqrt{T_{4}}, \\
\mathbf{W}_{2}=\frac{\left[1, \ldots, \frac{1}{N-1}\right]}{\sum_{i=1}^{N-1} \frac{i}{N-1}} .
\end{gathered}
$$

The formulas in this section provide a way to measure the divergence of a learned FM to a reference FM (and thus underlying ChI). However, the caveat is these indices are distance measures. As such, their values are not directly membership degrees. If one desires such information then a membership function needs to be specified or learned. 


\subsection{DATA-CENTRIC INDICES: $F_{\mathbf{D C}}\left(\mu, C_{\mu}, O\right)$}

In this section, we create new indices for answering data-driven questions. Ultimately, these results help us understand the diversity (or lack of) contained in $O$. Last, we explore a way to determine how much we should trust the result of fusion for data not in our training set. Meaning, have we seen its like before and how confident should we be in the result coming out of our system (fusion operator)?

\subsubsection{Variable Visitation}

In this subsection we address the question of how frequently is a FM variable encountered in training data? Algorithm 1 is a formal description of the procedure.

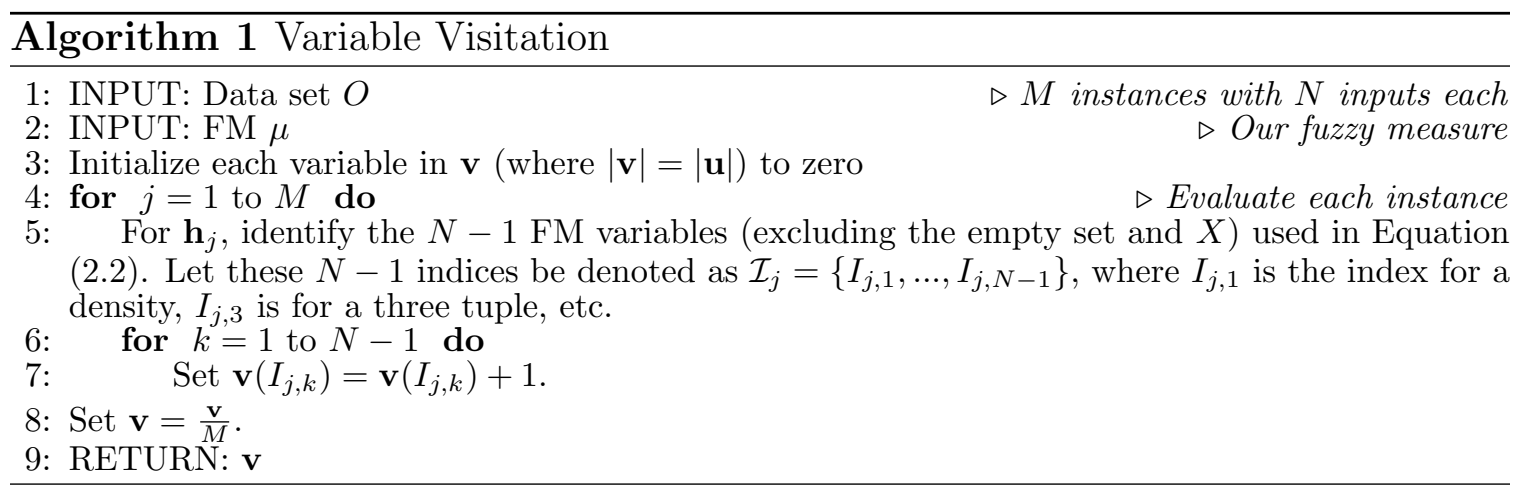

Algorithm 1 counts how many times a FM variable is encountered in the $M$ different $\mathrm{ChI}$ instances. It then normalizes these counts to obtain their relative frequency of occurrence per layer. Each layer has a different number of variables. Consider the case of $N=4$. Layer one (densities) has four variables and layer two (tuples) has six variables. Assume there are four training samples, $M=4$, so four walks. It is possible for each walk to touch a different density. However, these four walks can only touch four of the six tuples. We normalize per layer to provide an answer that supports what is the relative frequency of occurrence of variables at each layer, i.e., how often did I encounter this three tuple relative to other three tuples? Last, data supported variables are derived by looking at entries in $\mathbf{v}$ whose value is greater than 
zero. Data unsupported are entries in $\mathbf{v}$ with value (probability) zero.

\subsubsection{Percentage of Data Supported Variables}

The next question we address is overall, what percentage of our FM variables are supported by data? To answer this question we first run Algorithm 1. Next, we "harden" $\mathbf{v}$-meaning we convert $\mathbf{v}$ into $\hat{\mathbf{v}}$ such that an index in $\hat{\mathbf{v}}$ is 0 if its corresponding value in $\mathbf{v}$ is 0 , otherwise it is assigned a 1 . Next, we calculate an auxiliary variable,

$$
\delta_{1}=\sum_{k=1}^{|\hat{\mathbf{v}}|} \hat{\mathbf{v}}(k) .
$$

The answer to our question is

$$
i_{1}(\mu, O)=\frac{\delta_{1}}{|\hat{\mathbf{v}}|} \in[0,1]
$$

\subsubsection{Walk Visitation}

The last two subsections focused on individual variables. The next index (see Algorithm 22 we explore is based on the fact that the discrete (finite $X$ ) $\mathrm{ChI}$ is in reality $N$ ! LCS operators. As such, we need to know which of these operators are supported by training data.

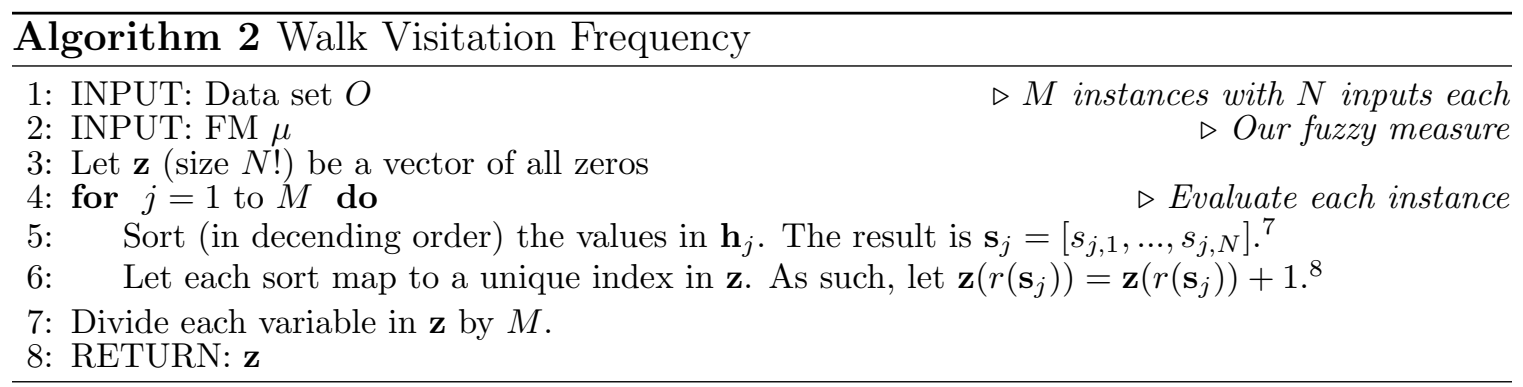

\footnotetext{
${ }^{8}$ For example, let $N=2$ and $h_{1}\left(\left\{x_{2}\right\}\right) \geq h_{1}\left(\left\{x_{1}\right\}\right)$. The sort index (walk) would therefore be $[2,1]$.

${ }^{8}$ Note, $r(\cdot) \in\{1, \ldots, N !\}$ is the index resolving function for sort $\mathbf{s}_{j}$.
} 
Algorithm 2 simply iterates each instance in our training data, determines its sort, and a mapping function (from sort to index in the FM) is used to increment the counts. At the end, these counts are normalized by the number of instances $(M)$.

\subsubsection{Percentage of LCSs Observed}

Algorithm 2 determines the relative frequency of occurrence for each walk. The next index is a summarizing statistic of what percentage of walks were encountered? This is calculated in a similar respect to index $i_{1}$. First, we convert $\mathbf{z}$ into a vector of zeros and ones $(\hat{\mathbf{z}})$. The index is then calculated as $i_{2}(\mu, O)=\frac{\delta_{2}}{N !} \in[0,1]$, where $\delta_{2}$ is the sum of the $\hat{\mathbf{z}}$ values.

\subsubsection{Dominant Walk Identification}

The next index asks the high-level summarizing question of, is our training data diverse, or does there exist a dominant walk? This index, $i_{3}(\mu, O)$, is

$$
i_{3}(\mu, O)=\max _{k} \mathbf{z}(k)
$$

If the training data has ideal walk variety then $\mathbf{z}$ is a uniform distribution. On the other hand, when diversity is poor we drive towards a single value of one and zero elsewhere. Thus, higher values for $i_{3}(\mu, O)$ mean less diversity in data and therefore our fusion solution may suffer.

\subsubsection{Should we Trust our Fused Result?}

The above techniques are focused on answering questions about variable and walk visitation and data diversity. However, at the end of the day we are typically given a set of data $O$, that may or may not be diverse, and we have to learn from what we are given. An important question is, if we are given a new instance ( $N$ inputs)-e.g., a sample from test data-can we trust the fused result? Whereas this might seem like a 
simple question, it is an important and fundamental one. Algorithm 3 is our proposed method.

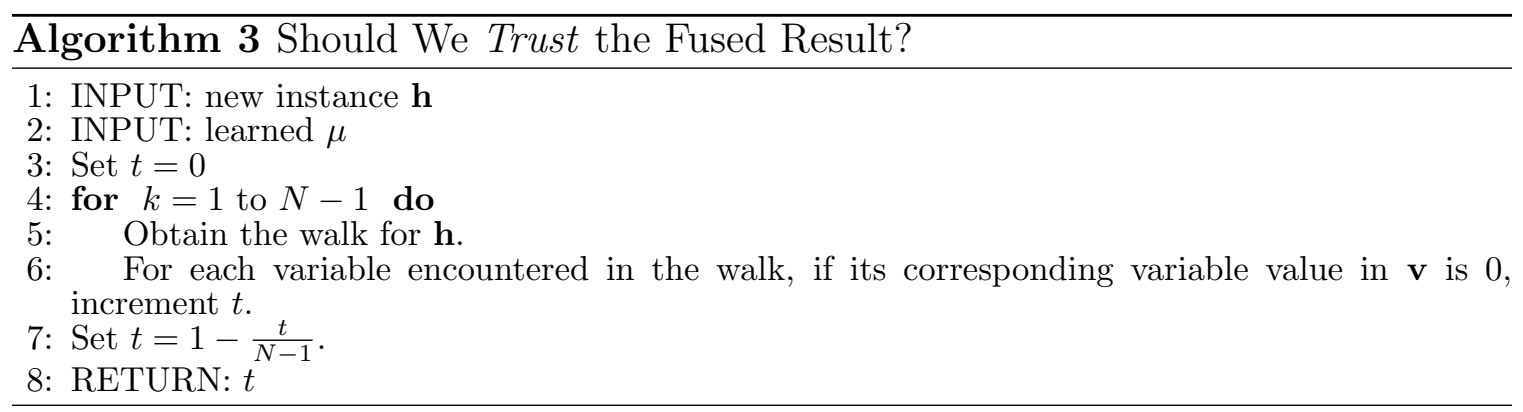

Algorithm 3 yields a value $t \in[0,1]$. This value is one when we have encountered data in training to support all of its relevant FM variables. However, as $t$ decreases, we are relying on variables (parts of our fusion calculation) that were not supported by data but are most likely a result of the optimization process. As we showed in [21], this often depends on the optimizer. For example, in simple quadratic programming data unsupported variables will take the lowest possible value to ensure monotonicity. Is this the "correct" value that should have been used? Most likely, no. The point is, a system can now return the tuple $\left(C_{\mu}(\mathbf{h}), t\right)$, i.e., what is the result of fusion and how much might we trust this in terms of what percentage of the answer was supported by data.

\subsection{EXPERIMENTS AND RESULTS}

Herein, we fuse four heterogeneous architecture DCNNs-CaffeeNet, GoogleNet, ResNet50 and ResNet101-on the UC Merced (UCM) remote sensing dataset. The UCM includes 21 classes that are a mix of objects and landcover (see Table 2.1). Each image chip is $256 \mathrm{x} 256$ in spatial size and each pixel is approximately $0.3 \mathrm{~m}$ ground sampling distance (GSD) spatial resolution. Some classes, e.g., harbor and parking lot, are complex compositions of sub-entities (boats and vehicles); while others are general structural patterns of shapes (e.g., intersection and baseball diamonds). In general, 
Table 2.1: UCM benchmark dataset

\begin{tabular}{|c|c|c|c|}
\hline Label & Class & Label & Class \\
\hline $\mathbf{1}$ & agricultural & $\mathbf{1 2}$ & intersection \\
\hline $\mathbf{2}$ & airplane & $\mathbf{1 3}$ & medium residential \\
\hline $\mathbf{3}$ & baseball diamond & $\mathbf{1 4}$ & mobile home park \\
\hline $\mathbf{4}$ & beach & $\mathbf{1 5}$ & overpass \\
\hline $\mathbf{5}$ & buildings & $\mathbf{1 6}$ & parking lot \\
\hline $\mathbf{6}$ & chaparral & $\mathbf{1 7}$ & river \\
\hline $\mathbf{7}$ & dense residential & $\mathbf{1 8}$ & runway \\
\hline $\mathbf{8}$ & forest & $\mathbf{1 9}$ & sparse residential \\
\hline $\mathbf{9}$ & freeway & $\mathbf{2 0}$ & storage tanks \\
\hline $\mathbf{1 0}$ & golf course & $\mathbf{2 1}$ & tennis court \\
\hline $\mathbf{1 1}$ & harbor & & \\
\hline
\end{tabular}

the variability and complexity of overhead imagery is immense as visual cues exist at multiple levels: fine-scale (e.g. airplane shapes, vehicle presence, etc.) to large-scale (e.g., road way configurations in overpasses versus intersections versus freeway).

The DCNNs were trained using our procedure outlined in [46], which includes transfer learning and data augmentation. The trained DCNNs are then used in a locked state, i.e., no further learning happens in DL during the fusion stage. The training of the DCNNs are done in five-fold, cross validation manner; such that we have 5 sets of $80 \%$ training and $20 \%$ testing for both datasets. Per DCNN fold, threefold CV fusion is used. An approximately equal number of samples are randomly selected per class across the fusion folds. This helps avoid scenarios where classes get zero samples.

The reader should refer to our prior research for a detailed discussion of how to learn a ChI per class/output neuron versus a shared ChI across all neurons 37. In this article, the goal is not to simply analyze the accuracy of ChI-based DCNN fusion, that was already demonstrated in [37]. Instead, our goal is to open the hood on the learned solutions and see if the XAI-ChI tools provide additional insight. Most 
Table 2.2: Accuracy of Individual NNs and their ChI Fusion

\begin{tabular}{|l|l|l|l|l|l|}
\cline { 2 - 6 } \multicolumn{1}{c|}{} & CaffeeNet & GoogleNet & RN50 & RN101 & ChI \\
\hline UCM & 0.974 & 0.980 & 0.986 & 0.985 & 0.990 \\
\hline PatternNet & 0.932 & 0.953 & 0.955 & 0.955 & 0.997 \\
\hline
\end{tabular}

figures reported in this section are illustrated using the "redblue" color map coding for visual simplicity-which means zero is blue, white is 0.5 , and 1.0 is red. Furthermore, in each figure the NNs have been assigned the indices CaffeeNet=1, GoogleNet=2, ResNet50=3 and ResNet101 $=4$. Table 2.2 reports the classification accuracy of the individual NNs and their fused result. As a further point of reference, we also ran the PatternNet data set and obtained the classification results reported in Table 2.2 $[\mathrm{X}]$. As Table 2.2 is clearly the more difficult case, with respect to fusion, we use the XAI-ChI tools on it for analysis.

\subsubsection{Measure-Centric Indices}

Figure 2.2 is the Shapley index. We cannot report all results as there are five cross validation folds with respect to the NNs, three fusion folds per NN fold, and 21 object classes. However, the trends are more-or-less consistent across all folds in the UCM data set. As such, we arbitrarily selected the first fold.

Figure 2.2 reveals a few interesting stories. First, classes 13 and 19 clearly indicate the existence of a (different) dominant NN. On the other hand, classes 1, 2, 4, and 6 say all four NNs have equal worth. Overall, there are no global trends-i.e., no consensus across classes about a single best $\mathrm{NN}$ or a single poor NN. If we listen to the Shapley, it seems to say that different classes require different fusions and all NNs are vital to achieving success. Next, we analyze the interaction index (Figure 2.3).

In Figure 2.3, class 1 indicates independence between all of the NNs. However, class 15 indicates positive interaction between ResNet50 and ResNet101, and moreor-less independence otherwise. Class 10 indicates strong negative interactions (re- 


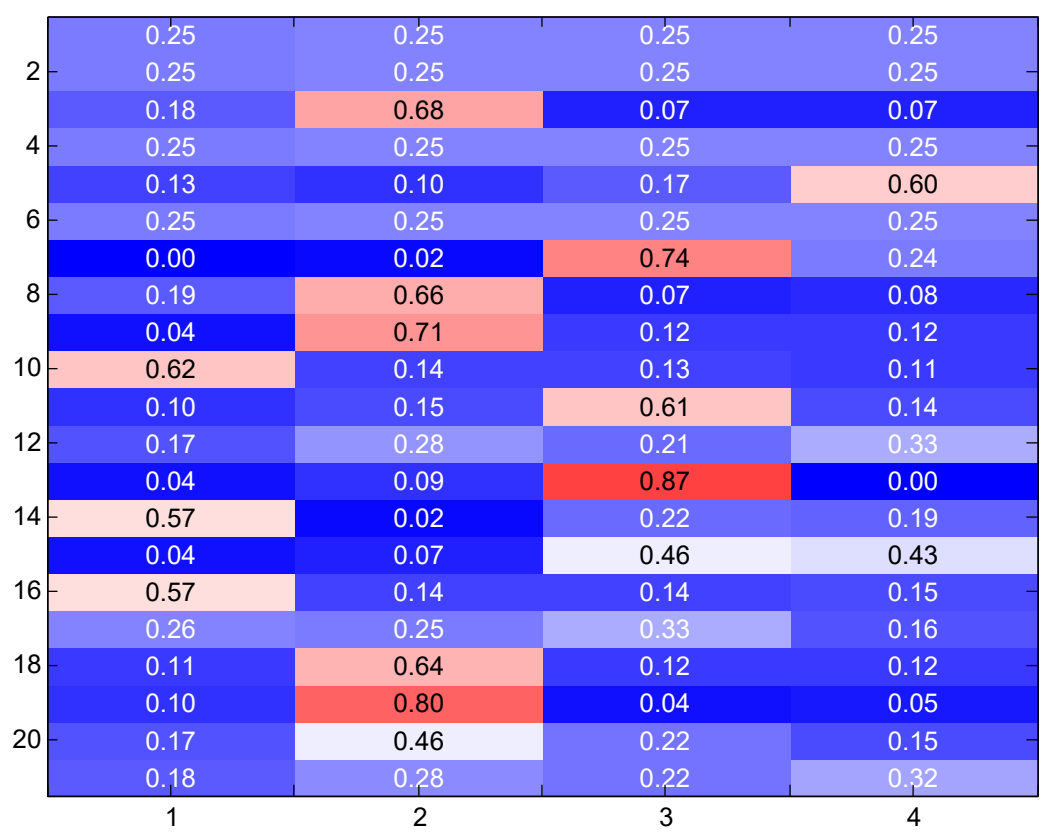

Figure 2.2: Visualization of the Shapley index values for (NN fold 1, fusion fold 1). Rows are the 21 classes and the $\mathrm{x}$-axis is the four NNs. Each row sums to one.
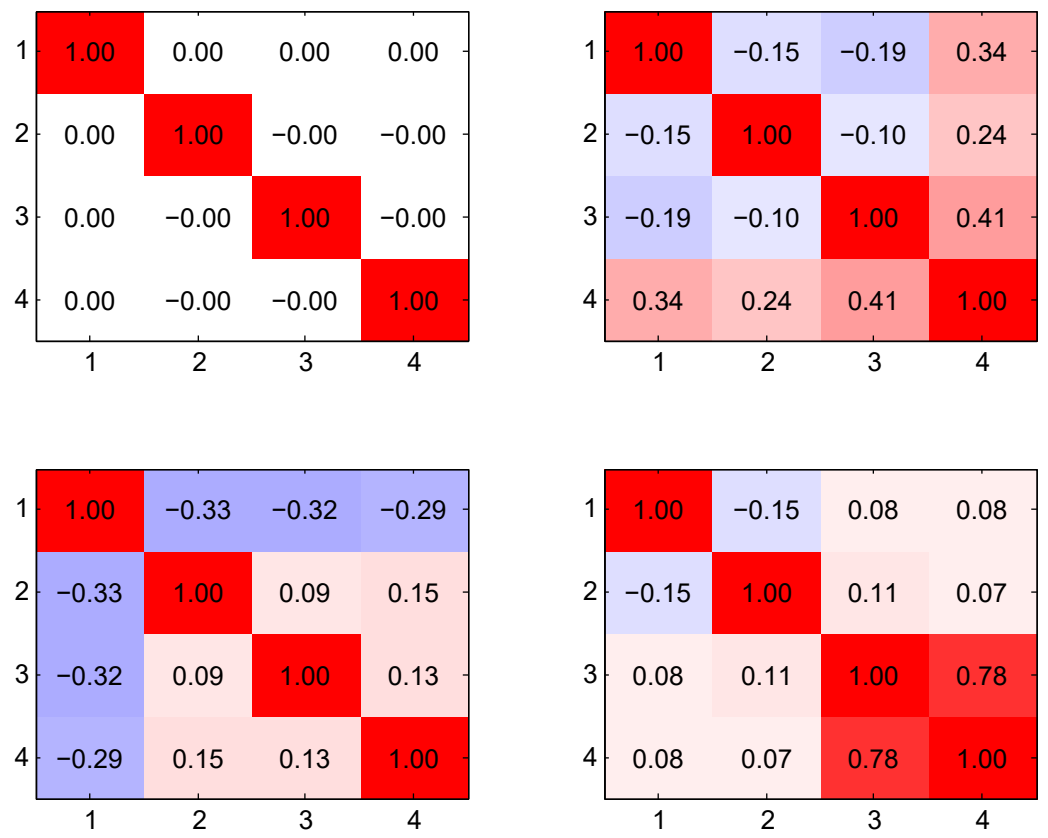

Figure 2.3: Visualization of the interaction index for (NN fold 1, fusion fold 1) and classes 1 (upper left), 5 (upper right), 10 (lower left) and 15 (lower right). Values range from -1 to 1 . 


\begin{tabular}{|c|c|c|c|}
\hline 0.17 & 0.20 & 0.10 & 0.00 \\
\hline 0.17 & 0.20 & 0.10 & 0.00 \\
\hline 0.25 & 0.17 & 0.30 & 0.31 \\
\hline 0.17 & 0.20 & 0.10 & 0.00 \\
\hline 0.26 & 0.15 & 0.29 & 0.24 \\
\hline 0.17 & 0.20 & 0.10 & 0.00 \\
\hline 0.15 & 0.31 & 0.31 & 0.37 \\
\hline 0.25 & 0.18 & 0.30 & 0.30 \\
\hline 0.25 & 0.20 & 0.35 & 0.33 \\
\hline 0.12 & 0.31 & 0.27 & 0.25 \\
\hline 0.25 & 0.15 & 0.26 & 0.25 \\
\hline 0.18 & 0.20 & 0.21 & 0.13 \\
\hline 0.21 & 0.26 & 0.36 & 0.43 \\
\hline 0.23 & 0.19 & 0.34 & 0.28 \\
\hline 0.30 & 0.11 & 0.37 & 0.25 \\
\hline 0.24 & 0.15 & 0.24 & 0.22 \\
\hline 0.16 & 0.23 & 0.28 & 0.14 \\
\hline 0.13 & 0.31 & 0.28 & 0.27 \\
\hline 0.18 & 0.29 & 0.34 & 0.39 \\
\hline 0.18 & 0.21 & 0.24 & 0.17 \\
\hline 0.19 & 0.18 & 0.17 & 0.08 \\
\hline 1 & 2 & 3 & 4 \\
\hline
\end{tabular}

Figure 2.4: Visualization of the indices for introspection for ( $\mathrm{NN}$ fold 1, fusion fold $1)$. Column one is $D_{1}(\max )$, two is $D_{2}(\min )$, three is $D_{3}$ (mean) and four is $D_{4}$ (LCOS).

dundancies) between CaffeeNet and the other three networks. While it is possible to study each class across all folds, what is apparent is variation across the interaction index results. There does not appear to be a NN that we can remove-or a NN that we can solely rely on.

\subsubsection{Integral-Centric Indices}

In this sub-section we investigate how the NNs are being combined via the ChI. Figure 2.4 is a visual summarization for NN fold 1 and fusion fold 1. Again, we focus on a single NN-fusion fold for sake of space.

The takeaway from Figure 2.4 is as follows. First, once again we see diversity in the way that different classes are aggregating their data. Classes 1, 2, 4, and 6 are for sure LCOS operators-as their fourth column distance values are zeros. However, they are not exactly min, max or mean like-confirmed by the other column one to three 

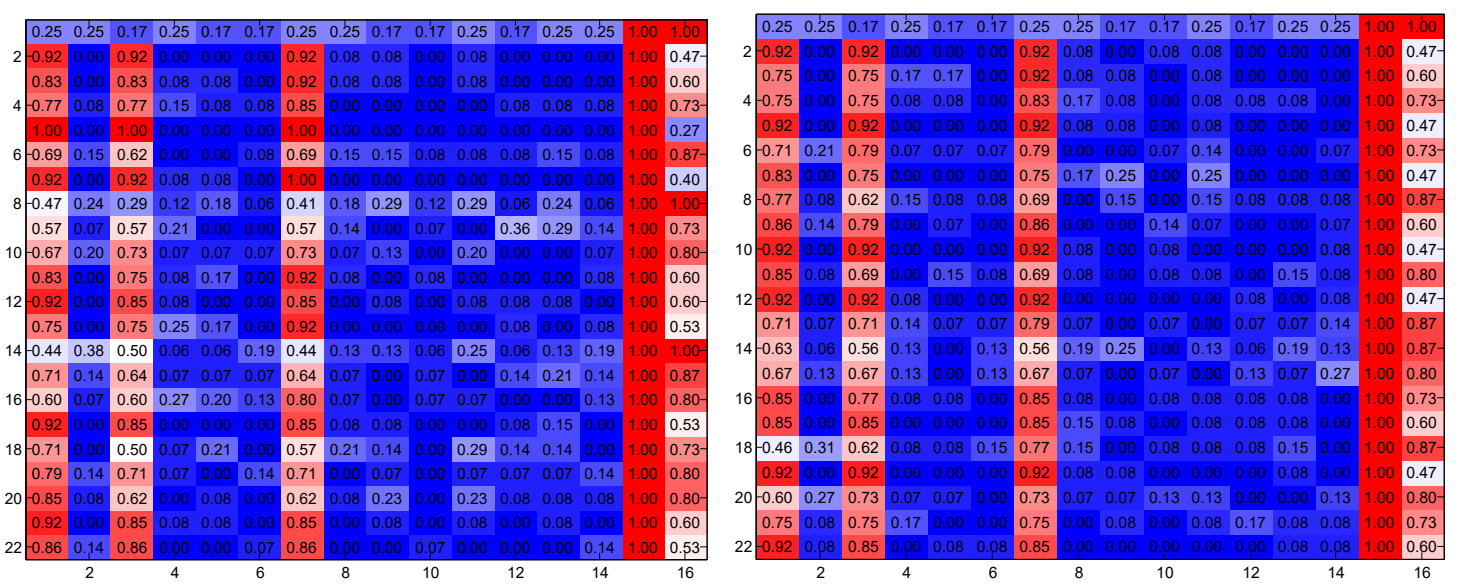

Figure 2.5: Visualization of variable visitation frequency and percentage of data supported variables for (left) (NN fold 1, fusion fold 1) and (right) (NN fold 2, fusion fold 1). Variables appear according their binary encoded index. Row 1 are ideal values-meaning, if the variable visitations were uniformly distributed then that is the value (and thus color) they should be. Row 2 is NN class 1, row 3 is NN class 2, and so forth. Columns 1 to 15 are the FM variable according their binary encoded index. For example, column 1 is variable $\left\{x_{1}\right\}$, column 2 is $\left\{x_{2}\right\}$, column 3 is $\left\{x_{1}, x_{2}\right\}$, and so forth. Column 16 is the percentage of data supported variables for each class (relative to color and decimal point display resolution).

values. If anything, they are the most like a mean operator. What is interesting is there are numerous non-LCOS like ChIs learned. This is encouraging per se because it helps to maybe justify that the ChI has a place here, versus a simpler fixed operator.

\subsubsection{Data-Centric Indices}

The summary of the last two sections is that there is variety in the worth of the individual NNs, their interactions and the aggregation operators. In this sub-section we explore the problem from a data-driven perspective to see if there is anything else to learn. Figure 2.5 shows variable visitation frequencies and percentage of data supported variables.

Figure 2.5 reveals a more disturbing trend in the UCM data set, as it relates to fusion. There is a strong imbalance in the visitation of variables and many low percentages of data supported variables. This indicates that variety is low in this 


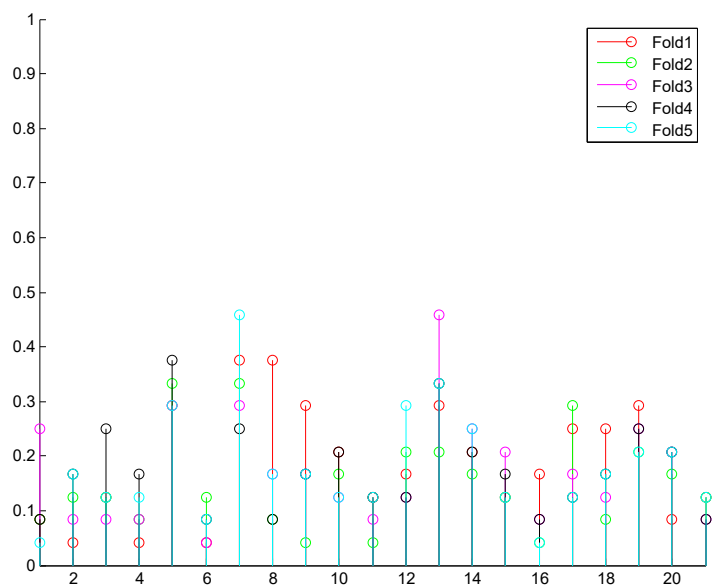

(a) Percentage of LCS operators observed (value one desired)

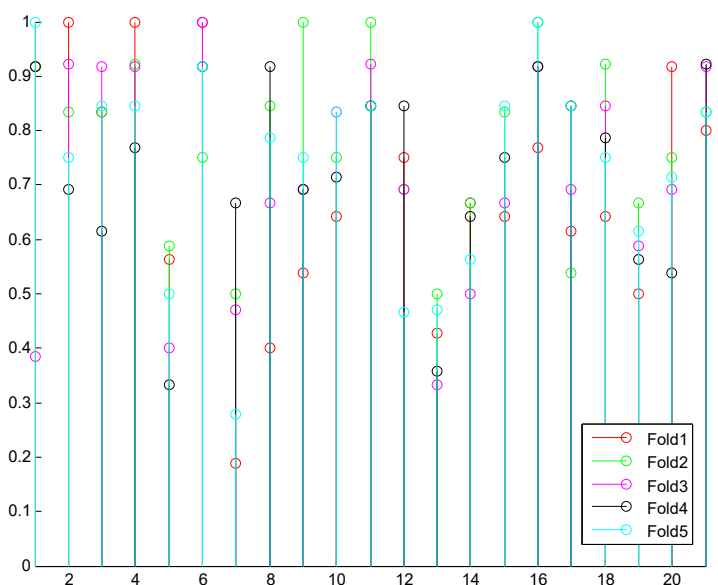

(b) Dominant walk index (lower values preferred)

Figure 2.6: (a) Percentage of LCS operators observed and dominant walk index. $\mathrm{X}$-axis is output neuron (class) number.

data set-again, with respect to fusion-and as such we do not really have adequate data to support learning a quality fusion solution. Figure 2.6 reinforces this claim in terms of observed percentage of LCSs and dominant walk assessment.

Figure 2.6 is alarming in the respect that most learned ChIs see approximately only ten to forty percent of the different underlying ChI LCSs. Furthermore, the index for dominant walk indicates that most NNs have a single dominant walk, more support in the claim of low data diversity.

Based on the alarming evidence from the data-driven indices, we went back to the data and looked at both the outputs (decisions) from the different NNs and the missclassification cases. What we discovered was two fold. First, the NNs are extremely strong classifiers, as indicated by their individual accuracy rates. As such, in the vast majority of cases they are all "voting" the same way. Second, most of the missed chips are either naturally ambiguous chips or mislabeled chips-e.g., a golf course with no golf features that was called forest (which would likely trick a human). As such, when they get something wrong they all more-or-less get it uniformly wrong. There is not much to work with in terms of fusion. Overall, there are numerous red flags that 
indicate a scenario of poor training data to support learning quality fusion solutions.

On a side note, the dominant walk imbalance seems to be related to the fact that we are fusing very strong learners. Meaning, a great number of inputs result in the same value across the NNs, a confidence of $100 \%$. As such, the default sort order becomes an ascending index ordering. Meaning, the dominant walk is really a product of the (too) strong agreement between the NNs and a further testament to low diversity.

\subsubsection{Measure + Integral + Data Index Combination}

In order to reach a holistic decision about the quality of our fusion solution, we need to combine the results of these three indices. The reality is these indices are obviously not independent of one another. The indices merely give us different ways to answer various questions. As such, the data-centric indices tell us that there is a severe lack of data diversity in the UCM data set. The Shapley, interaction index and aggregation indices tell us that there is diversity-meaning no obvious worthless DCNNs that can be removed. However, we would trust those indices a great deal if there was ideal data diversity. Since there is not, we must conclude that while we observe performance improvement on the UCM data set, there is not really the data to support the learning of fusion and based on what we can observe all DCNNs are needed.

\subsection{CONCLUSION AND FUTURE WORK}

In this article, we summarized existing indices and introduced new data-driven indices to support explainable AI (XAI) for the Sugeno Choquet integral. These indices were applied to the fusion of a set of heterogeneous architecture deep convolutional neural networks in remote sensing. The indices were used to assemble a more complete understanding of how fusion was working (or not working!). 
The data-driven indices put forth, and how we ultimately "use them", are preliminary. For example, in our remote sensing problem there were many folds and classes. There are likely better indices and/or better methods of summarizing the findings across folds to build a more complete and informative picture. Also, we are currently using these indices in a manual investigative mode. Our desire is to find new algorithmic ways to use these indices to improve the results. For example, it might be possible to use these indices during training to promote less over-fitting and more diverse NNs. 


\section{Chapter 3}

\section{EXPLAINABLE AI FOR THE CHOQUET INTEGRAL}

Since the dawn of computing, machines have been designed to carry out lists of deterministic operations given to them. In the last few decades, factors like Big Data and machine learning (ML) have given rise to a so-called data-driven era of artificial intelligence (AI). On one hand, we have data to help approximate parameters. On the other hand, the vast majority of algorithms are black box solutions. Sometimes, it might not be imperative to have an explanation as to what was learned; the solution in itself is all that is required. However, in other settings it may be vital to understand why a decision was reached. This need is a driving factor behind explainable

artificial intelligence (XAI). Another advantage of XAI is finding gaps in current AI to accelerate the field.

The big question in XAI is, what is the question! Specifically, what do we want from XAI and what constitutes an explanation? Common inquiries include: "what is the worth of our inputs?", "why did an AI make a particular decision?", "what evidence and process(es) were used?", "what confidence does our machine have in its decision?", "does our AI and/or data have a bias? (age, race, sex, etc.)", "what trust do we place in the machine and its decision", etc. There are many questions that make up XAI, some of which are specific to a particular tasks or tool, e.g. what features were learned by a convolutional neural net $(\mathrm{CNN})$ ?

At a high-level, XAI can be divided into two classes: methods that are naturally 
explainable from the ground up and methods that elicit an explanation post-learning. An example of "XAI by design" in computational intelligence is fuzzy logic. Often, IFTHEN rules are formed with meaningful linguistic variables whose values are modeled by fuzzy sets over appropriate domains. These rules are fired, aggregated, and mapped into linguistic expression. However, it is often the case that fuzzy logic learned from data might not be interpretable $[47,48,17]$. Another example of explainability in the fuzzy set community is linguistic summarization [49, 50]. Linguistic summaries are a high-level description provided for the user of the system. However, not all AI is created with explainability in mind. For instance, neural networks are not developed in this manner; this AI requires extracting any explanations after the model has been learned from data. Specifically, various methods have been developed to visually understand what a CNN is "looking at" in an image [51, 7, 52. There are numerous works and discussions about XAI in the literature. The reader can refer to [19] and [20] for a high-level overview and review of recent work.

Herein, we examine data/information fusion, a piece of the AI/ML puzzle. Namely, we focus on the fuzzy integral (FI). The FI is selected because it has been successfully applied across a variety of applications like computer vision [24], multi-criteria decision making [28, 29], multi-sensor fusion [22], remote sensing [53], neural networks [54], etc. The FI is a parametric function that yields a wide class of operators used in practice; the max, min, linear order statistics, and more. The FI aggregates data from sources-people, sensors, algorithms, and combinations thereof. Let $X=\left\{x_{1}, \ldots, x_{N}\right\}$ be $N$ sources and $h\left(x_{i}\right) \in \mathbb{R}$ is the input from source $i$. In general, an aggregation function is a mapping of data, $\mathbf{h}=\left\{h_{1}, \ldots, h_{N}\right\}$ (where $h_{i}=h\left(x_{i}\right)$ ), to data, i.e., $f_{\Theta}(\mathbf{h}) \rightarrow \mathbf{y}$, where $\Theta$ are model parameters. The FI is defined with respect to a capacity, which can be learned from data 21]. The focus of our article is XAI as it pertains to answering questions about aggregation.

There are many "types" of FIs; herein, we focus on the Choquet integral (ChI) 
[55], one of the most utilized types of FI. Furthermore, the ChI has many desirable properties, e.g., it recovers the Lebesgue integral for an additive/probability measure. There are many FIs, such as the Sugeno integral [39, 56], the non direct FI [31], the shape preserving FI [57], the general FI [58], the Shilkret integral [59], etc. A common thread across FIs is their underlying fuzzy measure (FM). In return, it is the FM that ultimately allows our proposed indices to be applicable to integrals beyond the $\mathrm{Ch}[$ The combination of the above is why we focus on a single integral, the ChI. Exploring different integrals and observing if there are any advantages of one over there other for XAI is beyond the scope of this article. This article aims to lay a foundation for such future work.

This paper makes the following specific contributions. First, we show that it is typical that a significant number of fusion variables are often not learned from data. As such, we identify which variables are data unsupported (referred to hereafter as missing). On this premise, we extend the Shapley (i.e. how important is each source) and the interaction index (i.e. how do sources interact) to data-driven partially observable domains. Next, we address the question "how good is a fusion decision" based on the learned fusion model. Last, we apply our XAI tools to synthetic data and real-world benchmark data in the context of classification from remote sensing to demonstrate and highlight their utility.

An overview of the paper is as follows (see Figure 3.1). In Section 3.1 we review the ChI, Sections 3.2, 3.3 and 3.4 highlight existing XAI tools and our new extensions, and last, Section 3.5 explores the fusion of different deep learners.

\subsection{MEASURE AND CHOQUET INTEGRAL}

On a discrete (finite $X$ ) domain, the fuzzy measure (FM), $\mu: 2^{X} \rightarrow \mathbb{R}^{+}$, is a function that satisfies the following two properties: (i) (boundary condition) $\mu(\emptyset)=0$, and

\footnotetext{
${ }^{1}$ The core principles are the same, however other integrals might result in different data observed FM variables. As a result, the formulas could change.
} 


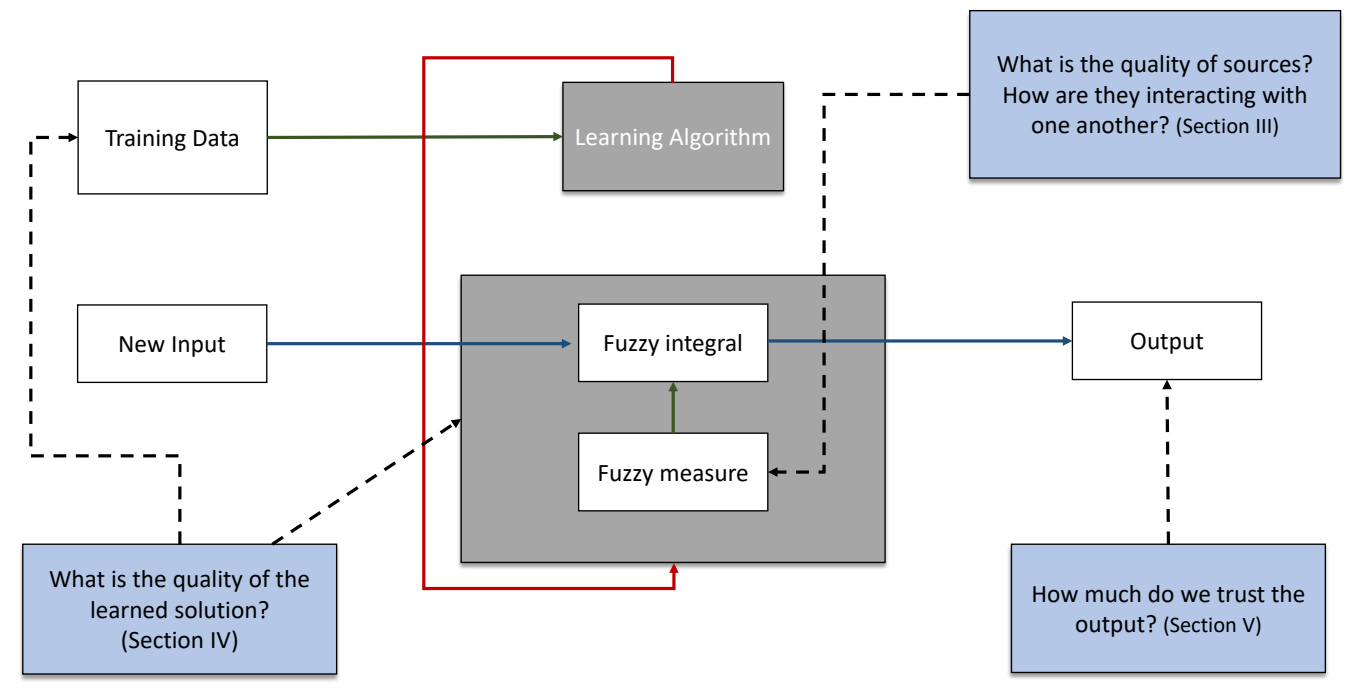

Figure 3.1: Depiction of the XAI questions asked herein and their respective locations in the overall fusion process.

(ii) (monotonicity) if $A, B \subseteq X$, and $A \subseteq B$, then $\mu(A) \leq \mu(B)^{2}$. One way to think about the FM is that it models the "interactions" (e.g., statistical correlations) between subsets of sources.

Let $h\left(x_{i}\right) \in \mathbb{R}^{\geq 0}$ be the input from source $i$. The ChI of $\mathbf{h}=\left(h_{1}, h_{2}, \ldots, h_{N}\right)^{t}$, where $h_{i}=h\left(x_{i}\right)$, is

$$
\int \mathbf{h} \circ \mu=\sum_{j=1}^{N} h_{\pi(j)}\left(\mu\left(A_{\pi(j)}\right)-\mu\left(A_{\pi(j-1)}\right)\right),
$$

for $A_{\pi(j)}=\left\{x_{\pi(1)}, \ldots, x_{\pi(j)}\right\}, \mu\left(A_{\pi(0)}\right)=0$, and $\pi$ such that $h_{\pi(1)} \geq h_{\pi(2)} \geq \ldots$ $\geq h_{\pi(N)}$. Once the FM is defined, the ChI becomes a specific aggregation operator. For example, the ChI becomes the max operator for $\mu(A)=1, \forall A \in X$, excluding $\mu(\emptyset)$ which is 0 . One useful way to discuss the ChI is in terms of $N$ ! linear convex sums (LCSs). Relative to a particular sort of the data $\left(\pi_{i}\right)$-of which there are $N$ !

\footnotetext{
${ }^{2}$ Sometimes a normality condition is imposed such that $\mu(X)=1$.
} 
possible sorts-the $\mathrm{ChI}$ is

$$
\sum_{j=1}^{N} h_{\pi_{i}(j)}\left(\mu\left(A_{\pi_{i}(j)}\right)-\mu\left(A_{\pi_{i}(j-1)}\right)\right)=\mathbf{h}_{\pi_{i}}^{t} \mathbf{w}_{\pi_{i}}
$$

where $w_{\pi_{i}}(j)=\mu\left(A_{\pi_{i}(j)}\right)-\mu\left(A_{\pi_{i}(j-1)}\right)$. For the ChI, these $N \times N$ ! weights are tied to the underlying $2^{N} \mathrm{FM}$ variables. The next section addresses one way in which these variables can be defined.

\subsubsection{Optimization of the ChI}

In this section, one method for determining the ChI is discussed. Most of the time it is impractical to have an expert define the FM; as the number of variables increases, the likelihood of arriving at a meaningful FM is not highly probable. Another route is to just determine the values of each of the singletons (densities) and to use a formula to calculate the remaining variables; e.g., the Sugeno $\lambda$-FM or the S-Decomposable FM [39]. While there are many methods to optimize the ChI (see $60,61,62,33$, 63, 64, 34, 65, 66, 67), we limit our scope to the following data-driven method. Full mathematical detail and experiments can be found in 21.

Let $O=\left\{\mathbf{h}_{j}, y_{j}\right\}, j=1, \ldots, M$, be $M$ training examples; where $\mathbf{h}_{j}$ is the $j$-th instance with data/information from $N$ inputs and $y_{j}$ is the ground-truth (e.g., class label, regression output, etc.) for $\mathbf{h}_{j}$. The sum of squared error (SSE) for $O$ is

$$
E(O, \mathbf{u})=\sum_{j=1}^{M} e_{j}^{2}=\sum_{j=1}^{M}\left(\mathbf{c}_{j}^{T} \mathbf{u}-y_{j}\right)^{2}=\|D \mathbf{u}-\mathbf{y}\|_{2}^{2}
$$

where $\mathbf{u}=\left[\mu\left(\left\{x_{1}\right\}\right), \ldots, \mu\left(\left\{x_{1}, x_{2}\right\}\right), \mu\left(\left\{x_{1}, x_{3}\right\}\right), \ldots, \mu(X)\right]$ (lexiographic vector of size $\left.2^{N}-1\right), D=\left[\begin{array}{llll}\mathbf{c}_{1} & \mathbf{c}_{2} & \ldots & \mathbf{c}_{M}\end{array}\right]^{T}$ (full dataset), $\mathbf{y}=\left[\begin{array}{llll}y_{1} & y_{2} & \ldots & y_{M}\end{array}\right]^{T},\|\cdot\|_{2}$ is norm-2 operation, and $\mathbf{c}_{j}$ holds the coefficients of $\mathbf{u}$ for observation $\mathbf{h}_{j}$, e.g., for $N=3$ and 


$$
\begin{aligned}
& h\left(x_{2}\right) \geq h\left(x_{1}\right) \geq h\left(x_{3}\right)^{3}, \\
& \quad c=\left[0, h\left(x_{2}\right)-h\left(x_{1}\right), 0, h\left(x_{1}\right)-h\left(x_{3}\right), 0,0, h\left(x_{3}\right)\right] .
\end{aligned}
$$

The regularized SSE optimization problem is

$$
\min _{\mathbf{u}} f(\mathbf{u})=\|D \mathbf{u}-\mathbf{y}\|^{2}+\beta v(\mathbf{u})
$$

where $\beta \in \mathbb{R}^{\geq 0}$ is a regularization constant (which balances the "cost" (or penalty) of obtaining minimum function error relative to our desire to have minimal model complexity) and $v(\mathbf{u})$ is an index of model complexity (e.g., k-additive and Mobius, Gini-Simpson, $\ell_{p}$-norm, etc. [40]), subject to the FM boundary and monotonicity conditions (see 41] for how to pack the constraints into a linear algebra expression), which can be solved via quadratic programming. Full code and explanation (including how to build the constraint matrix $C$ ) can be found at https://github.com/BMur/ChoquetIntegral.

\subsubsection{Data Supported and Unsupported Variables}

A benefit of working with the ChI is knowing how training data maps to ChI parameters. That is, we can identify unsupported variables. In [21], we showed that the sort operation in a discrete ChI reveals which FM variables are exercised. For sort $\pi_{i}$, we get $w_{\pi_{i}}(j)=\mu\left(A_{\pi_{i}(j)}\right)-\mu\left(A_{\pi_{i}(j-1)}\right)$. For example, let $N=3$ and let $h_{2}>h_{3}>h_{1}$. The FM variables that are encountered are $\mu\left(h_{2}\right), \mu\left(\left\{h_{2}, h_{3}\right\}\right)$ and $\mu\left(\left\{h_{1}, h_{2}, h_{3}\right\}\right)$. By taking the entire training set of data into account, we can identify which of the variables are never encountered. Herein, we will exploit this property to build our XAI tools. It is our opinion that the ChI is a wonderful framework for XAI because the math is explainable by design, and as we show below, tools exist to tie

\footnotetext{
${ }^{3}$ The dimensionality of variables are as follows: $\mathbf{h}_{j} \in \mathcal{R}^{N}, y_{j} \in \mathcal{R}, \mathbf{u} \in \mathcal{R}^{2^{N}-1}$, and $c \in R^{2^{N}-1}$
} 
these properties to what was extrapolated from data.

\subsection{MEASURE CENTRIC INDICES}

Numerous researchers have created indices to help explain the ChI, but on the basis that the full measure/ChI has been observed. However, as we showed, this is not often the case for data-driven fusion. Two of the most common indices are the Shapley index [68] and the interaction index [42]. The Shapley index assigns a value to each input to describe its worth; whereas, the interaction index computes how much two inputs interact (are they redundant, independent, or complimentary?). However, to date, all have assumed that the FM was fully obtainable, i.e. all variables were explicitly learned or determined. Herein, we propose new indices that do not have this inherent missing information bias.

\subsubsection{Shapley Index}

\section{XAI Question: How good is a source?}

The Shapley index is a way to summarize the $2^{N}$ variables in the FM. Generally, they are interpreted to be the worth of the different sources. The Shapley index is

$$
\begin{aligned}
\Phi_{\mu}(i) & =\sum_{K \subseteq X \backslash\{i\}} \zeta_{X, 1}(K)(\mu(K \cup\{i\})-\mu(K)), \\
\zeta_{X, 1}(K) & =\frac{(|X|-|K|-1) !|K| !}{|X| !}
\end{aligned}
$$

where $K \subseteq X \backslash\{i\}$ denotes all proper subsets from $X$ that do not include source $i$. The Shapley values of $\mu$ is the vector $\Phi_{\mu}=\left[\Phi_{\mu}(1), \ldots, \Phi_{\mu}(N)\right]^{t}$ where $\sum_{i=1}^{N} \Phi_{\mu}(i)=1$. The Shapley index can be interpreted as the average amount of contribution of source $i$ across all coalitions. Intuitively, it is a weighted sum of the difference between the ensemble not including source $i$ and the ensemble including source $i$. To further 
illustrate the Shapley index, a numerical example is provided (see Table 3.1). This example is built on the ChI presented in Figure 3.2 .

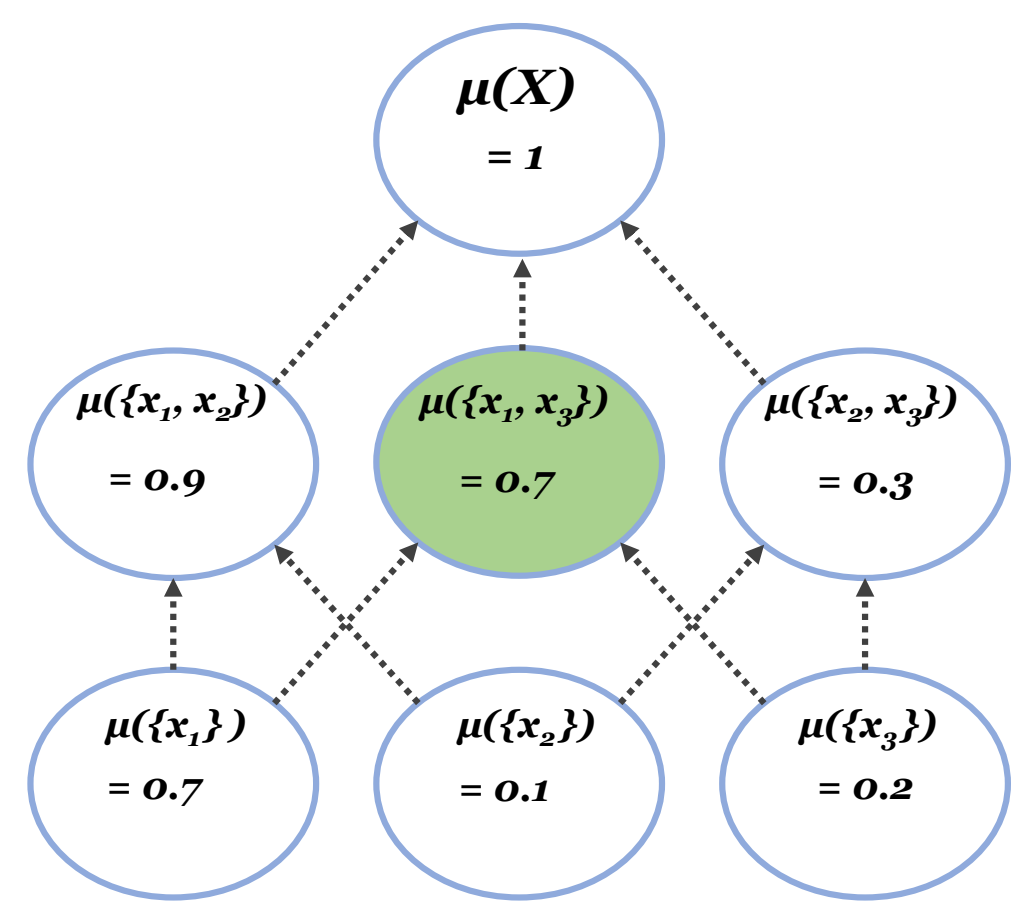

Figure 3.2: Visualization of an example $\mathrm{FM}$ for $\mathrm{N}=3$. All variables are observed except $\mu(x 1, x 3)$ (green).

Table 3.1: Shapley index for FM shown in Figure 2.

\begin{tabular}{|ll|}
\hline$\Phi_{\mu(1)}$ & $.68=\frac{1}{3}(.7-0)+\frac{1}{6}(.9-.1)+\frac{1}{6}(.7-.2)+\frac{1}{3}(1-.3)$ \\
{$[0.5 \mathrm{pt} / 3 \mathrm{pt}] \Phi_{\mu(2)} .18=\frac{1}{3}(.1-0)+\frac{1}{6}(.9-.7)+\frac{1}{6}(.3-.2)+\frac{1}{3}(1-.7)$} \\
{$[0.5 \mathrm{pt} / 3 \mathrm{pt}] \Phi_{\mu(3)} .13=\frac{1}{3}(.2-0)+\frac{1}{6}(.7-.7)+\frac{1}{6}(.3-.1)+\frac{1}{3}(1-.9)$}
\end{tabular}

\subsubsection{Walk Centric Shapley}

XAI: How good is a source relative to what we observed?

The Shapley index can be modified in order to exclude any variables that are not data supported. Hereafter, we refer to each input sort $\left(\pi_{i}\right)$ as a walk. The FM can be visualized as a Hasse Diagram, where nodes are the FM variables and edges are the 
monotonicity constraints. Furthermore, a layer in the Hasse Diagram is all variables of equal size cardinality, i.e., layer 1 is $\left\{\mu\left(\left\{x_{1}\right\}\right), \mu\left(\left\{x_{2}\right\}\right), \ldots\right\}$, layer 2 is all tuples, etc. In the $\mathrm{ChI}$, each input sort results in selecting one variable in each layer. Equation (3.6a) is the Walk-Centric Shapley index,

$$
\begin{aligned}
\bar{\Phi}_{\mu}(i) & =\sum_{K \subseteq X \backslash\{i\}} \zeta_{X, 2}(K)(\mu(K \cup\{i\})-\mu(K)), \\
\zeta_{X, 2}(K) & =\frac{(|X|-|K|-1) !|K| !}{|X| !} \mathbb{1}_{(K \cup\{i\})} \mathbb{1}_{(K)}, \\
\widetilde{\Phi}_{\mu}(i) & =\frac{\bar{\Phi}_{\mu}(i)}{\sum_{j=1}^{N} \bar{\Phi}_{\mu}(j)} .
\end{aligned}
$$

Extending the Shapley index required including the indicator function, $\mathbb{1}$, which takes the value of 1 if the FM value is data-supported and 0 otherwise. The Shapley index values sum to 1 , which helps with our interpretation of their relative worth degrees. Herein, we normalize the Walk Centric Shapley index in order to maintain the same semantics.

The calculation of the Walk Centric Shapley index, relative to Figure 3.2, is provided in Table 3.2. The variable, $\mu\left(\left\{x_{1}, x_{3}\right\}\right)$, is data-unsupported. As such, computation on any term containing this variable is not considered. For example, in $\Phi_{\mu}(1)$ (first row of Table 3.2 , the third term is 0 because it contains the $\mu\left(\left\{x_{1}, x_{3}\right\}\right)$. The reader can see that a single missing variable leads to a noticeable change of the second source's worth from 0.18 to 0.08 . The point is, unsupported variables change the way that one thinks about worth relative to what is observable.

\subsubsection{Interaction Index}

\section{XAI: How are two sources interacting?}

Next, we explore the interaction index, which informs us about how well two sets of sources interact with one another. Similar of the Shapley index, the interaction 
Table 3.2: Walk Centric Shapley for Figure 1.

\begin{tabular}{|lc|}
\hline$\Phi_{\mu}(1)$ & $.6=\frac{1}{3}(.7-0)+\frac{1}{6}(.9-.1)+(0)+\frac{1}{3}(1-.3)$ \\
{$[0.5 \mathrm{pt} / 3 \mathrm{pt}] \Phi_{\mu}(2)$} & $.08=\frac{1}{3}(.1-0)+\frac{1}{6}(.9-.7)+\frac{1}{6}(.3-.2)+(0)$ \\
{$[0.5 \mathrm{pt} / 3 \mathrm{pt}] \Phi_{\mu}(3)$} & $.13=\frac{1}{3}(.2-0)+(0)+\frac{1}{6}(.3-.1)+\frac{1}{3}(1-.9)$ \\
{$[0.5 \mathrm{pt} / 3 \mathrm{pt}] \widetilde{\Phi}_{\mu}(1)$} & $.74=\frac{.6}{.81}$ \\
{$[0.5 \mathrm{pt} / 3 \mathrm{pt}] \widetilde{\Phi}_{\mu}(2)$} & $.09=\frac{.08}{.81}$ \\
{$[0.5 \mathrm{pt} / 3 \mathrm{pt}] \widetilde{\Phi}_{\mu}(3)$} & $.16=\frac{.13}{.81}$ \\
\hline
\end{tabular}

index assumes a fully observable FM. The interaction index [42] between $i$ and $j$ is

$$
\begin{aligned}
\mathcal{I}_{\mu}(i, j) & =\sum_{K \subseteq X \backslash\{i, j\}} \zeta_{X, 3}(K)(\mu(K \cup\{i, j\}) \\
& -\mu(K \cup\{i\})-\mu(K \cup\{j\})+\mu(K)), \\
\zeta_{X, 3}(K) & =\frac{(|X|-|K|-2) !|K| !}{(|X|-1) !},
\end{aligned}
$$

where $\mathcal{I}_{\mu}(i, j) \in[-1,1], \forall i, j \in\{1,2, \ldots, N\}$. A value of 1 (respectively, -1 ) represents the maximum complementary (respectively, redundancy) between $i$ and $j$. A

\begin{tabular}{|c|c|}
\hline $\mathcal{I}_{\mu}(1,2)$ & $.15=\frac{1}{2}(.9-.7-.1+0)+\frac{1}{2}(1-.7-.3+.2)$ \\
\hline$[0.5 \mathrm{pt} / 3 \mathrm{pt}] \mathcal{I}_{\mu}(1,3)$ & $-.15=\frac{1}{2}(.7-.7-.2+0)+\frac{1}{2}(1-.9-.3+.1)$ \\
\hline$[0.5 \mathrm{pt} / 3 \mathrm{pt}] \mathcal{I}_{\mu}(2,3)$ & $.05=\frac{1}{2}(.3-.1-.2+0)+\frac{1}{2}(1-.9-.7+.7)$ \\
\hline
\end{tabular}
numerical example is provided in Table 3.3 with respect to Figure 3.2 .

Table 3.3: Interaction index for Figure 2.

\subsubsection{Walk-Centric Interaction}

XAI: How are two sources interacting, relative to what we observed? 
Next, we extend the interaction index to listen only to data supported variables.

$$
\begin{aligned}
& \widetilde{\mathcal{I}}_{\mu}(i, j)=\sum_{K \subseteq X \backslash\{i, j\}} \zeta_{X, 4}(K)(\mu(K \cup\{i, j\}) \\
&-\mu(K \cup\{i\})-\mu(K \cup\{j\})+\mu(K)), \\
& \zeta_{X, 4}(K)=\frac{\widehat{\zeta}_{X, 4}(K)}{\sum_{\widehat{K} \subseteq X \backslash\{i, j\}} \widehat{\zeta}_{X, 4}(\widehat{K})} \\
& \widehat{\zeta}_{X, 4}(K)=\frac{(|X|-|K|-2) !|K| !}{(|X|-1) !} \\
& \mathbb{1}_{(K \cup\{i\})} \mathbb{1}_{(K \cup\{j\})} \mathbb{1}_{(K \cup\{i, j\})} \mathbb{1}_{(K)} .
\end{aligned}
$$

See Table IV for a numeric example of the walk-centric interaction index with respect to Figure 2.

Table 3.4: Walk-centric interaction index for Figure 2.

\begin{tabular}{lr}
$\widetilde{\mathcal{I}_{\mu}}(1,2)$ & $.1=(.9-.7-.1+0)+(0)$ \\
{$[0.5 \mathrm{pt} / 3 \mathrm{pt}] \widetilde{\mathcal{I}_{\mu}}(1,3)$} & $-.1=(0)+(1-.9-.3+.1)$ \\
{$[0.5 \mathrm{pt} / 3 \mathrm{pt}] \widetilde{\mathcal{I}_{\mu}}(2,3)$} & $0=(.3-.1-.2+0)+(0)$ \\
\hline
\end{tabular}

Remark 1. In Section 3.2.4 we extended the interaction index to partially observable domains with respect to two sources. In [69], Grabisch extended the interaction index to $\mathcal{I}(A, B)$, where $A, B \subseteq X$. Our missing variable weighting (Equation (3.8a)) naturally extends to interaction between sets of sources.

\subsection{DATA-CENTRIC INDICES}

Oftentimes computational methods are applied to a data set based on intuition of the practitioner without any type of analysis of the data. Whereas Section 3.2 produced metrics that explain characteristics in and across the sources, this section's focus is 
insights into the quality of a learned ChI to a set of training data. We specifically address the data, directly, to determine their diversity. In this section, we present a set of indices that describes a different aspect of data diversity. However, understanding the diversity is only half of the story; we also present a new index to assess the degree to which we should trust a new sample's fused result.

\subsubsection{Variable Visitation}

\section{XAI: How often was a FM variable observed?}

Each of our indices are a different piece of evidence in the XAI big picture. The idea behind the next index (see Algorithm 4) is to determine the frequency for which a FM variable is observed in data

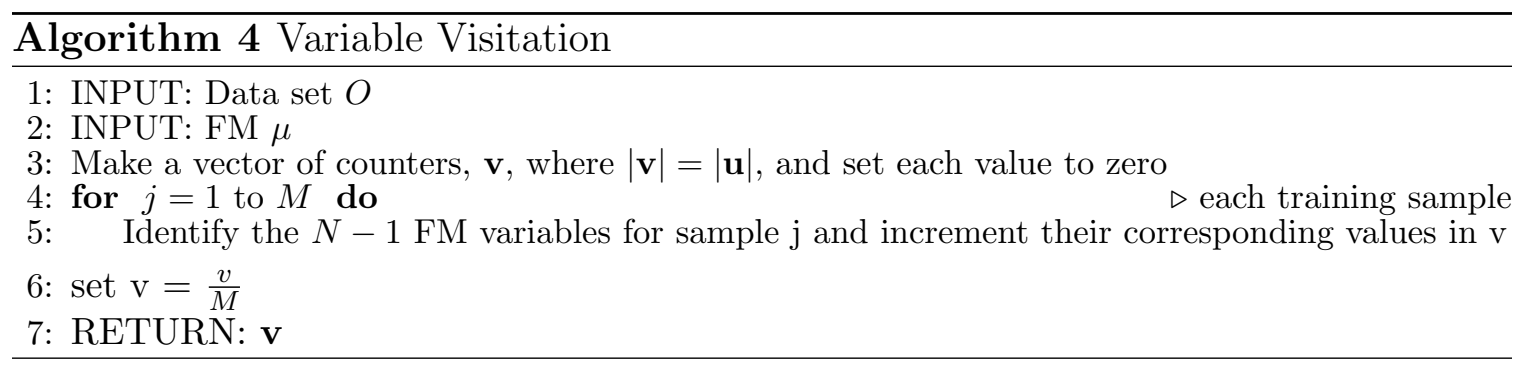

Table $\mathrm{V}$ is a numeric example for $N=3$. The first column shows the $M=4$ samples. Column two is the different FM variables. Column 3 is the number of times each variable was encountered. The next two columns are our proposed variable visitation index and its conversion to a binary seen/not seen.

\subsubsection{Percentage of Data Supported Variables}

\section{XAI: How many FM variables were observed?}

While knowing the relative frequency of visibility of each FM variable per layer is useful, it may still be too much information for a user to interpret. The next index reduces the $2^{N}$ probabilities into a single value that tells us total percentage of the variables seen. To this end, our variable visitation probabilities are "hardened." 
Table 3.5: Numerical example of the variable visitation and percentage of data supported variables indices.

\begin{tabular}{ccccc}
\hline Samples & FM Variables & Count & $\mathbf{v}$ & $\hat{\mathbf{v}}$ \\
\hline$\left\{x_{1}, x_{2}, x_{3}\right\}$ & $\left\{x_{1}\right\}$ & 2 & .50 & 1 \\
$\left\{x_{2}, x_{1}, x_{3}\right\}$ & $\left\{x_{2}\right\}$ & 1 & .25 & 1 \\
$\left\{x_{3}, x_{1}, x_{2}\right\}$ & $\left\{x_{3}\right\}$ & 1 & .25 & 1 \\
$\left\{x_{1}, x_{3}, x_{2}\right\}$ & $\left\{x_{1}, x_{2}\right\}$ & 2 & .5 & 1 \\
& $\left\{x_{1}, x_{3}\right\}$ & 2 & .5 & 1 \\
& $\left\{x_{2}, x_{3}\right\}$ & 0 & 0 & 0 \\
\hline
\end{tabular}

First, we convert $\mathbf{v}$ into $\hat{\mathbf{v}}$ such that an index in $\hat{\mathbf{v}}$ is 0 if its corresponding value in $\mathbf{v}$ is 0 , otherwise it is assigned a 1 . We calculate the number of nonzero observed probabilities,

$$
\delta_{1}=\sum_{k=1}^{|\hat{\mathbf{v}}|} \hat{\mathbf{v}}(k) .
$$

The percentage of data supported variables index is

$$
i_{1}(\mu, O)=\frac{\delta_{1}}{|\hat{\mathbf{v}}|} \in[0,1] .
$$

Following the previous example from Table 3.5 , approximately $83 \%$ of the variables are supported.

\subsubsection{Walk Visitation}

\section{XAI: What contexts did we observe?}

The data-centric indices proposed thus far have provided valuable insights to the inner workings of a learned FM. However, each input/sample has a corresponding 
walk, which corresponds to one of the $N$ ! LCS operators. Algorithm 5 exploits this property to provide the walk visitation frequency.

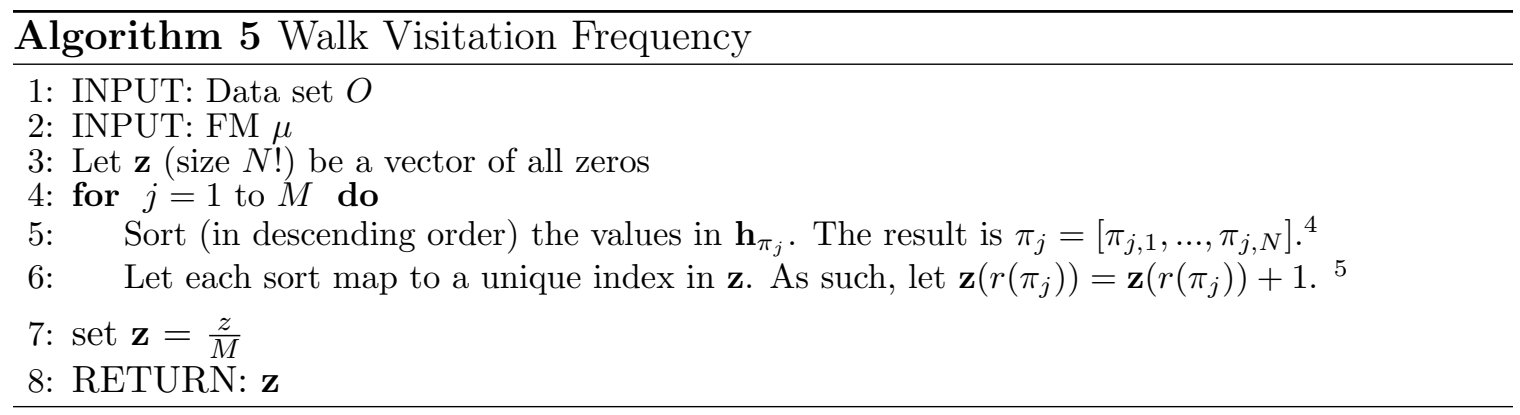

Algorithm 5 sorts each instance of the training data, and then it uses a mapping function to increment the number of times each sort has been seen. Once each iteration is complete, the values are normalized by the number of training instances.

\subsubsection{Percentage of Walks Observed}

\section{XAI: What percentage of contexts were observed?}

Algorithm 5 provides insight into the relative frequency of how often each walk is encountered. Next, we outline an index that tells us what percentage of walks are seen. However, that again is information overload as there are $N$ ! walks. Similar to $i_{1}, z$ is "hardened." The percentage of LCSs observed is $i_{2}(\mu, O)=\frac{\delta_{2}}{N !} \in[0,1]$, where $\delta_{2}$ is the sum of the $\hat{\mathbf{z}}$ values. This index can be calculated based on the example provided in Table 3.5. The percentage of walks seen is $\frac{4}{6}$.

\subsubsection{Dominant Walk Identification}

\section{XAI: Are there any anomalous contexts?}

The next index tells us about the diversity of the data. The idea behind this index is to look for irregularly high observation walks. It is computed as

${ }^{4}$ For example, let $N=2$ and $h\left(x_{2}\right) \geq h\left(x_{1}\right)$. The sort index (walk) would therefore be $[2,1]$.

${ }^{5}$ Note, $r(\cdot) \in\{1, \ldots, N !\}$ is the index resolving function for sort $\mathbf{s}_{j}$. 


$$
i_{3}(\mu, O)=\max _{k} \mathbf{z}(k)
$$

In theory, a balanced data set would have a uniform distribution for the probability of walk frequencies. As we discuss in the Section 3.5 , cases where $i_{3}(\mu, O)$ is large are suspicious and need further analysis.

\subsubsection{Complexity Analysis}

Here, we provide a succinct analysis of the computational complexity of the proposed indices. It is trivial to show that the computational complexity of the Shapley index is $O\left(N 2^{N-1}\right)$, one difference term per monotonicity constraint. In the case of the WalkCentric Shapley, complexity simply grows by a factor of 2 (the indicator functions) and the Walk-Centric Interaction index grows by a factor of 4 (the indicator functions). With respect to the data-centric indices, computing the walks and keeping track of the data-supported variables only requires running a sort on the $M$ data points. The point is, the proposed indices do not come at any significant computational complexity, namely relative to the fact that they are often an offline procedure.

\subsection{TRUST: POTENTIAL VARIABILITY INDEX}

\section{XAI: How much trust do we place in a ChI decision?}

The above sections focus on answering XAI questions about learning. In this section, we address the question of how much should we trust the output of a ChI? Specifically, we focus on the viewpoint of how many missing parameters were used in a particular ChI instance. Ideally, we would like this value to be low, which corresponds to a low interval of uncertainty in that instance. To the best of our knowledge, this has not been tackled before with respect to the FI. Let $\mathbf{h}_{\pi_{i}}$ be the sorted input and $\mathbf{W}_{\mathbf{h}}$ be its corresponding walk. For example, if $N=4$ and $h_{4} \geq$ $h_{2} \geq h_{1} \geq h_{3}$ then $\mathbf{W}_{\mathbf{h}}=\left(\left\{x_{4}\right\},\left\{x_{2}, x_{4}\right\},\left\{x_{2}, x_{2}, x_{4}\right\}, X\right)$. Each FM variable that was 
encountered during training is assigned a real-valued number during optimization. On the other hand, each missing variable has interval-valued uncertainty [21]. That is, each missing variable has an interval of uncertainty for a $\mathrm{ChI}$ instance because of the monotonicity constraints. The missing variable can take any value between the max of its corresponding variables at layer L-1 and the min of the corresponding variables at layer $\mathrm{L}+1$. Algorithm 6 outlines how to calculate the potential variability index.

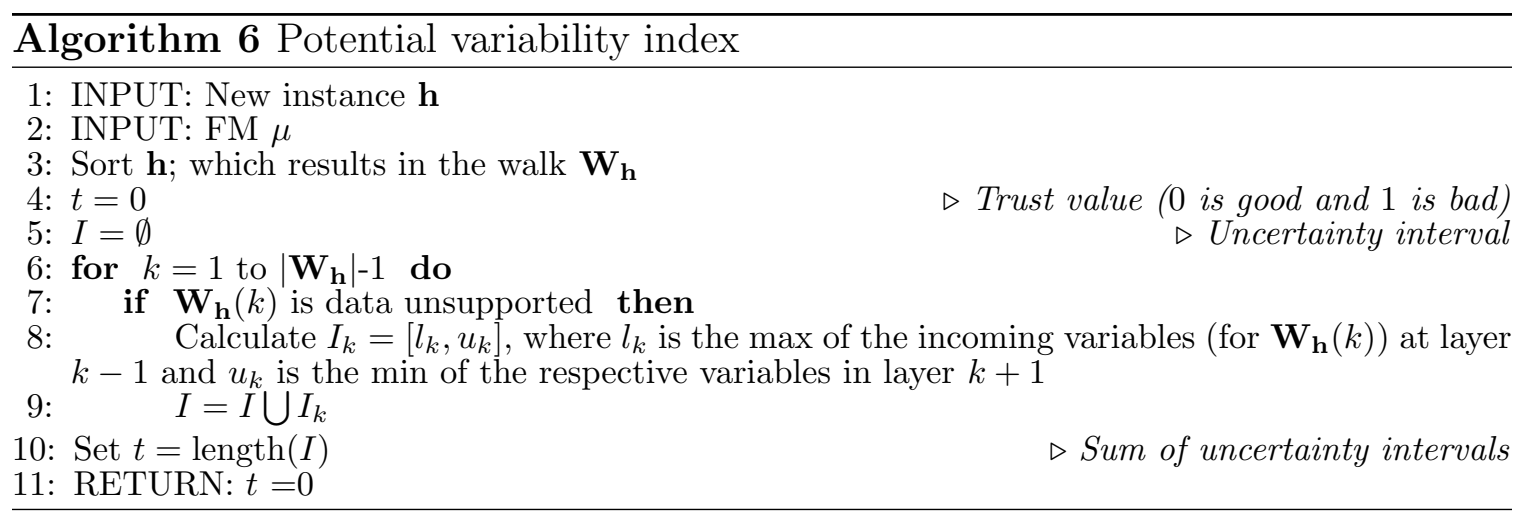

It should be noted that a low $t$ does not guarantee that the ChI output is correct. It only informs us that data were sufficient to support the learning algorithm. The index $t$ is merely evidence to warn a user when decisions are being made that were not encountered during training.

\subsection{EXPERIMENTS}

In order to understand how the proposed indices work in a real-world setting, we use the ChI to fuse a set of heterogeneous architecture deep convolutional neural networks (DCNNs) for object detection and land classification in remote sensing. Herein, we fuse seven DCNNs — CaffeNet [70], GoogleNet [71], ResNet 50 [72], ResNet 101, DenseNet [73], InceptionResNetV2 [74], and Xception [75] - on the AID remote sensing data set $[76]$. The AID data set contains 10,000 images of 30 different aerial scene types (see Table 3.6). 
Table 3.6: Summary of the AID data set

\begin{tabular}{|ll|ll|}
\hline Types & \# images & Types & \# images \\
\hline airport & 360 & mountain & 340 \\
bare land & 310 & park & 350 \\
baseball field & 220 & parking & 390 \\
beach & 400 & playground & 370 \\
bridge & 360 & pond & 420 \\
center & 260 & port & 380 \\
church & 240 & railway station & 260 \\
commercial & 350 & resort & 290 \\
dense residential & 410 & river & 410 \\
desert & 300 & school & 300 \\
farmland & 370 & sparse residential & 300 \\
forest & 250 & square & 330 \\
industrial & 390 & stadium & 290 \\
meadow & 280 & storage tanks & 360 \\
medium residential & 290 & viaduct & 420 \\
\hline
\end{tabular}

The DCNNs were trained following the procedures outlined in [46], which included state-of-the-art steps like transfer learning, data augmentation, drop out, and regularization. For fusion, the DCNNs were locked, which means no further training occurs in the networks. The DCNNs were trained using five-fold cross validation, meaning we have 5 sets and $80 \%$ of the data was used for training and $20 \%$ for testing. Per DCNN fold, two-fold cross validation is used for fusion. An approximately equal number of samples are randomly selected per class across the fusion folds to ensure that each class is represented in each of the folds. The reader can refer to [37] for a more detailed discussion of how to train the ChI; one per class or a shared ChI across classes.

Herein, it is not our goal to reiterate the already demonstrated accuracy of the ChI for DCNN fusion (see Table 3.7 and 37 ). Instead, our goal is to use the proposed XAI indices to shed light into what was learned. 
Table 3.7: Fusion Improvement on AID data set

\begin{tabular}{ccccc}
\hline & CaffeNet & GoogleNet & ResNet 50 & ResNet 101 \\
\hline Accuracy & 0.9395 & 0.9528 & 0.9580 & 0.9589 \\
\hline & DenseNet & InceptionResNetV2 & Xception & ChI \\
\hline Accuracy & 0.9480 & 0.9484 & 0.9622 & $\mathbf{0 . 9 8 2 1}$ \\
\hline
\end{tabular}

Table 3.8: Shapley index for AID data set: Shapley value (rank order value)

\begin{tabular}{cllllllll}
\hline Class & Shapley index & Caffe & Google & Res 50 & Res 101 & Dense & InceptionRes V2 & Xception \\
\hline \multirow{2}{*}{1} & All variables & $0.063(1)$ & $0.087(3)$ & $0.088(4)$ & $0.076(2)$ & $0.094(5)$ & $0.221(6)$ & $\mathbf{0 . 3 6 9 ( 7 )}$ \\
& DS variables & $0.049(1)$ & $0.071(3)$ & $0.075(4)$ & $0.064(2)$ & $0.088(5)$ & $0.230(6)$ & $\mathbf{0 . 4 2 1}(\mathbf{7})$ \\
\hline \multirow{2}{*}{6} & All variables & $0.043(1)$ & $0.071(4)$ & $0.045(2)$ & $0.122(5)$ & $0.135(6)$ & $0.068(3)$ & $\mathbf{0 . 5 1 2 ( 7 )}$ \\
& DS variables & $0.033(2)$ & $0.062(4)$ & $0.027(1)$ & $0.097(5)$ & $0.124(6)$ & $0.061(3)$ & $\mathbf{0 . 5 9 3 ( 7 )}$ \\
\hline \multirow{2}{*}{28} & All variables & $0.097(2)$ & $0.132(5)$ & $0.113(4)$ & $0.102(3)$ & $0.095(1)$ & $\mathbf{0 . 3 1 9 ( 7 )}$ & $0.138(6)$ \\
& DS variables & $0.063(1)$ & $0.114(5)$ & $0.094(3)$ & $0.095(4)$ & $0.080(2)$ & $\mathbf{0 . 4 3 3 ( 7 )}$ & $0.118(6)$ \\
\hline \multirow{2}{*}{30} & All variables & $0.120(3)$ & $0.161(5)$ & $0.126(4)$ & $\mathbf{0 . 1 9 7 ( 7 )}$ & $0.107(2)$ & $0.182(6)$ & $0.105(1)$ \\
& DS variables & $0.140(4)$ & $0.193(6)$ & $0.069(1)$ & $0.148(5)$ & $0.097(2)$ & $\mathbf{0 . 2 4 1 ( 7 )}$ & $0.109(3)$ \\
\hline
\end{tabular}

\subsubsection{Shapley Index-Based Discoveries}

Due to the high number of experiments (five neural network cross validation folds, two fusion folds, and 30 object classes), all of the results are not reported. Instead, we report a few interesting discoveries (Table 3.8).

As shown in Section 3.2, missing variables can have a large impact on our understanding of a source's worth. Table 3.8 highlights a few instances from the AID data set where a source's worth fluctuates as much as $10 \%$ based on the classical Shapley definition versus our Walk-Centric Shapley formulation. Furthermore, it is worth highlighting that this fluctuation results in a different rank ordering of sources based on their individual worth.

\subsubsection{Potential Variability Index-Based Discoveries}

In this experimental section, there is not a "ground truth" per se. As such, our goal was to explore how our potential variability index affects the ability of fusion to compute an accurate output. In Figure 3.3, values for the potential variability 
(horizontal-axis) and cost/penalty (vertical-axis) for samples from three different arbitrary classes are provided, where the cost is the confidence of the classifier and negative values represent missed classifications. Ideally, we would like to see a value of one on the vertical-axis with a potential variability of zero (aka, all variables are supported). However, Figure 3.3 informs us that a low potential variability index does not guarantee an accurate cost value. This concept is demonstrated by class 26 (blue); as many points have a low cost despite having zero potential variability. On the other hand, a high potential variability does not mean a low cost. Class 8 (green) contains several samples that have a high potential variability while maintaining a high cost value. It is often said, garbage in, garbage out. Fusion is not guaranteed to solve every problem. If each DCNN says it is not the correct class, fusion will not fix that. For example, this index helped us discover that class 26 had errors because of the classifiers, not because there are data unsupported variables.

\subsubsection{Data-Centric Index-Based Discoveries}

Next, the data-centric indices exposed additional weaknesses. Table 3.9 highlights the percentage of data supported variables, number of walks visited, percentage of walks observed, and the frequency of the top two most visited walks. Ideally, we would hope that the percentage of walks observed would be one and that the frequency of each walk would be equal. However, the reader can see that at most $2 \%$ of walks are observed and two walks typically dominate our training data. This is concerning because it tells us that there is a dangerously low amount of variety in our training data to aid learning a quality fusion solution. In one case, the top two walks account for over $80 \%$ of the data. 


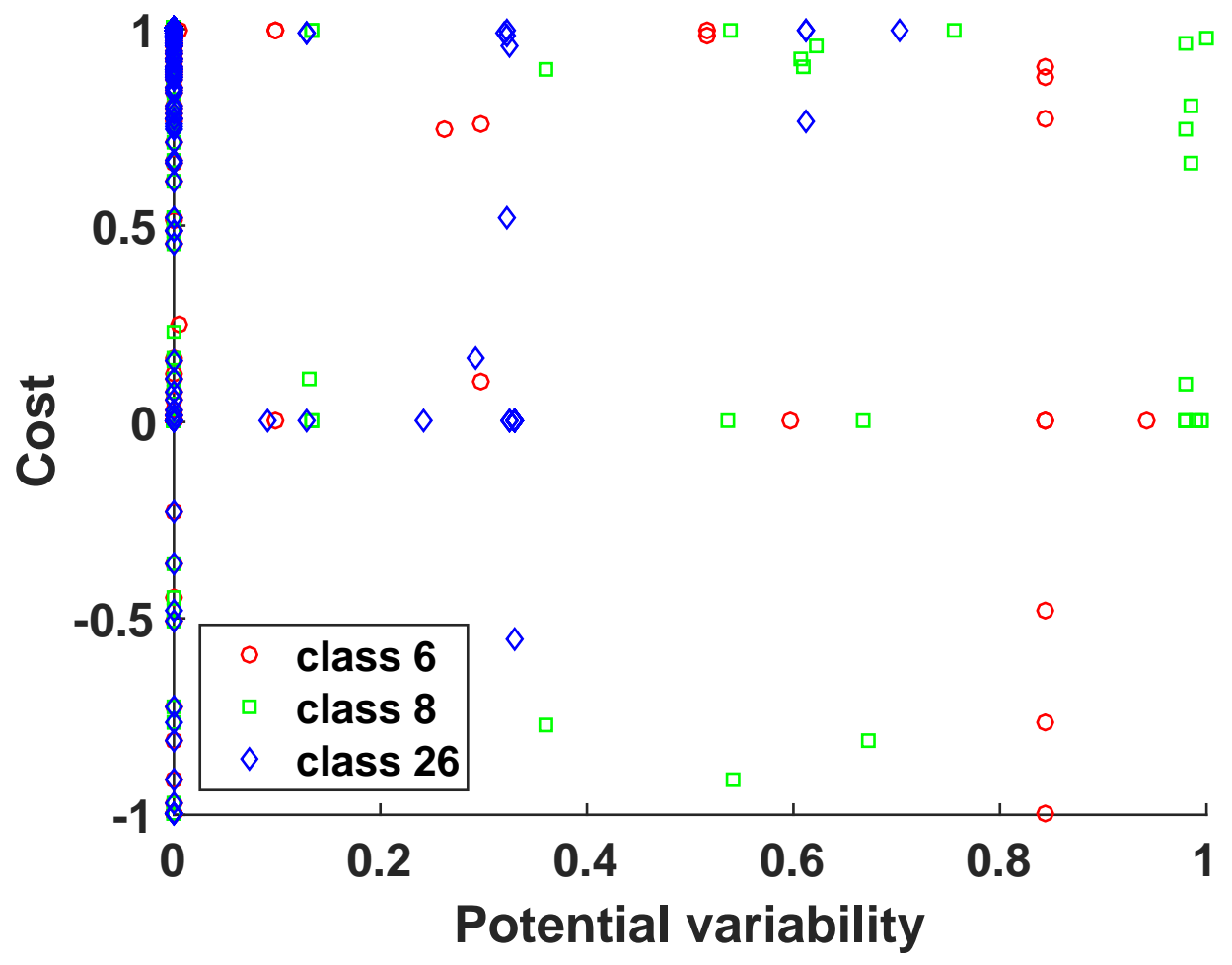

Figure 3.3: Potential variability for classes, 6, 8, and 28.

\subsubsection{Overall XAI assessment}

The proposed indices need to be combined to garner a complete picture of the quality of our fusion solution. The reality is, these indices are not independent of one another. At a high-level, the indices highlight that we have dangerously low variety as it relates to learning a trustworthy fusion solution. Furthermore, the Shapley and interaction indices highlight that there are no definitive DCNNs that can be eliminated, especially when we look across classes. This is promising for fusion, meaning there is no single dominant network and fusion is likely needed and beneficial.

These XAI observations prompted us to investigate the dominant walks. The most frequently encountered sort order is $1,2,3$, etc. The reason this occurs is because all the networks are producing the same value, e.g., all zeros, ones, etc. Our analysis has led us to believe that our DCNNs are too strong of classifiers - they almost always say the same thing, right or wrong. As a result, we should explore weaker learners 
and simultaneously learning of nets alongside fusion. This is not alarming, it is a strategy used in areas like ensemble learning.

In summary, only a very small part of our fusion solution was learned and as a result, it is likely that future scenarios can arise and impact the generalization of our model.

Table 3.9: Data-centric indices for DCNN fusion on the AID data set

\begin{tabular}{|c|c|c|c|c|c|c|}
\hline Class & $\begin{array}{c}\text { Percentage of } \\
\text { data supported } \\
\text { variables }\end{array}$ & $\begin{array}{c}\text { Number of } \\
\text { walks visited }\end{array}$ & $\begin{array}{l}\text { Percentage of } \\
\text { LCS observed }\end{array}$ & $\begin{array}{l}\text { Frequency of } \\
\text { top walk }\end{array}$ & $\begin{array}{l}\text { Frequency of } \\
\text { walk-2 }\end{array}$ & $\begin{array}{l}\text { Frequency of } \\
\text { top two walks }\end{array}$ \\
\hline 1 & 0.780 & 55 & 0.011 & 0.385 & 0.310 & 0.696 \\
\hline 2 & 0.638 & 39 & 0.008 & 0.411 & 0.382 & 0.793 \\
\hline 3 & 0.567 & 32 & 0.006 & 0.373 & 0.317 & 0.689 \\
\hline 4 & 0.622 & 36 & 0.007 & 0.475 & 0.317 & 0.792 \\
\hline 5 & 0.701 & 50 & 0.010 & 0.376 & 0.313 & 0.689 \\
\hline 6 & 0.701 & 49 & 0.010 & 0.298 & 0.277 & 0.575 \\
\hline 7 & 0.709 & 56 & 0.011 & 0.335 & 0.309 & 0.644 \\
\hline 8 & 0.740 & 59 & 0.012 & 0.341 & 0.309 & 0.651 \\
\hline 9 & 0.677 & 50 & 0.010 & 0.465 & 0.250 & 0.715 \\
\hline 10 & 0.591 & 38 & 0.008 & 0.415 & 0.304 & 0.719 \\
\hline 11 & 0.654 & 42 & 0.008 & 0.338 & 0.285 & 0.623 \\
\hline 12 & 0.449 & 22 & 0.004 & 0.462 & 0.332 & 0.794 \\
\hline 13 & 0.803 & 70 & 0.014 & 0.361 & 0.282 & 0.643 \\
\hline 14 & 0.441 & 25 & 0.005 & 0.561 & 0.244 & 0.805 \\
\hline 15 & 0.709 & 42 & 0.008 & 0.393 & 0.334 & 0.728 \\
\hline 16 & 0.567 & 34 & 0.007 & 0.438 & 0.300 & 0.738 \\
\hline 17 & 0.512 & 31 & 0.006 & 0.462 & 0.332 & 0.794 \\
\hline 18 & 0.772 & 67 & 0.013 & 0.373 & 0.280 & 0.653 \\
\hline 19 & 0.669 & 42 & 0.008 & 0.458 & 0.267 & 0.726 \\
\hline 20 & 0.732 & 57 & 0.011 & 0.402 & 0.260 & 0.662 \\
\hline 21 & 0.701 & 47 & 0.009 & 0.442 & 0.274 & 0.716 \\
\hline 22 & 0.756 & 57 & 0.011 & 0.339 & 0.285 & 0.624 \\
\hline 23 & 0.819 & 90 & 0.018 & 0.405 & 0.219 & 0.623 \\
\hline 24 & 0.795 & 59 & 0.012 & 0.341 & 0.303 & 0.644 \\
\hline 25 & 0.890 & 96 & 0.019 & 0.301 & 0.264 & 0.565 \\
\hline 26 & 0.591 & 37 & 0.007 & 0.428 & 0.369 & 0.797 \\
\hline 27 & 0.850 & 82 & 0.016 & 0.363 & 0.270 & 0.632 \\
\hline 28 & 0.559 & 35 & 0.007 & 0.407 & 0.331 & 0.738 \\
\hline 29 & 0.512 & 32 & 0.006 & 0.343 & 0.330 & 0.673 \\
\hline 30 & 0.370 & 23 & 0.005 & 0.464 & 0.307 & 0.770 \\
\hline
\end{tabular}




\subsection{CONCLUSION AND FUTURE WORK}

In this article, we proposed metrics to address different questions about a learned fusion. Specifically, we proposed indices to assess the quality of a model, quality of our sources and their interactions, and how much we should trust a particular output of the ChI. We demonstrated analytical cases and the benchmark AID remote sensing data set was used as a case study. Each index plays a pivotal role in understanding and diagnosing weaknesses in fusion. Furthermore, our extension of the Shapley and interaction indices enable a more accurate assessment based on what was observed from training data.

While this paper is a step in the right XAI direction, it merely provides evidence via different indices. As we showed in the Section 3.5, these indices should ideally be combined by an algorithm or expert to make even more useful high-level conclusions. In future work, we will seek additional mathematics/algorithms to infer and build linguistic summarizations. Another research avenue is to formally take the exposed weaknesses and to fold them into the optimization process. For example, we observed a potential flaw due to independently training DCNNs. A next step is to use our indices to help promote the simultaneous training of a set of diverse learners to increase performance. 


\section{Chapter 4}

\section{INFORMATION FUSION-2-TEXT: EXPLAINABLE AGGRE- GATION VIA LINGUISTIC PROTOFORMS}

\subsection{INTRODUCTION}

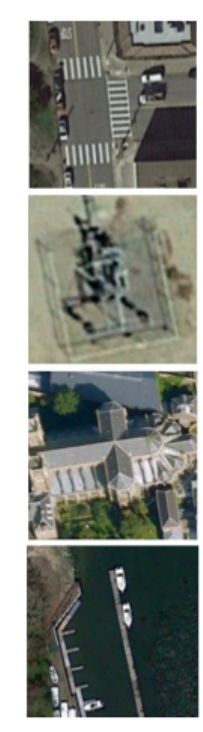

Training Data

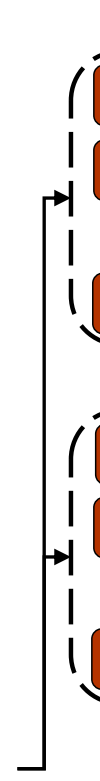

Fold 1

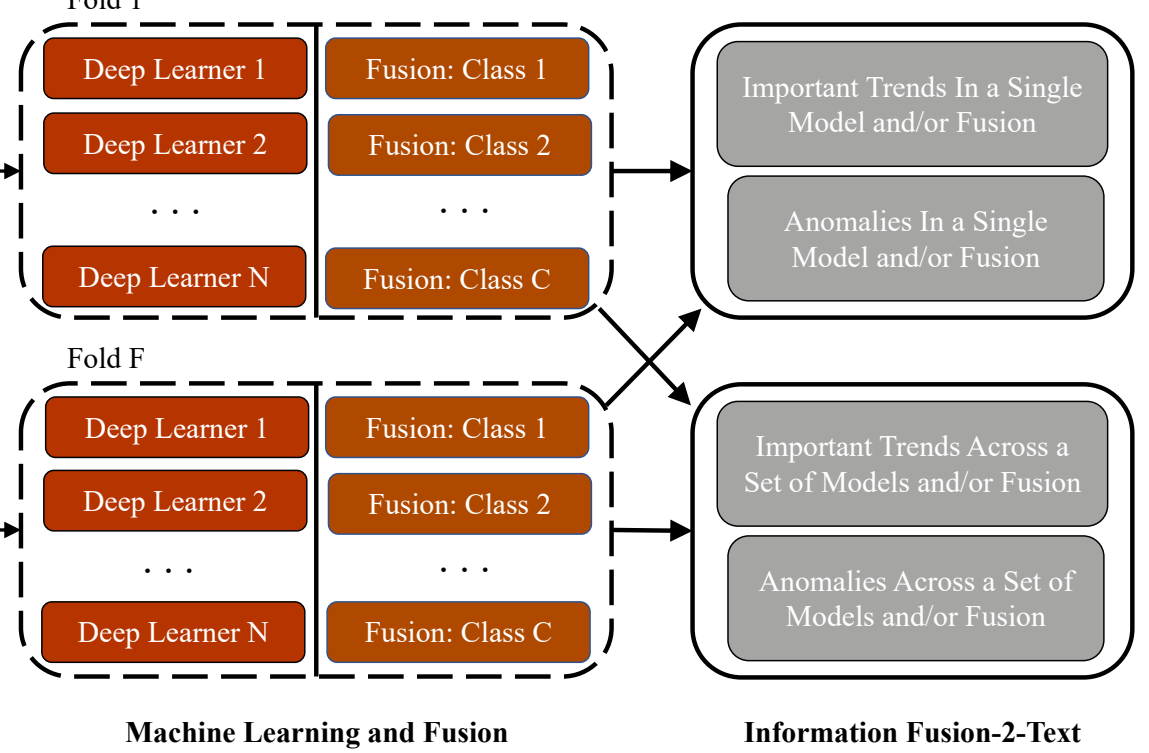

Figure 4.1: Graphical illustration of fusion-2-text for the case study explored herein involving object detection and land cover classification in remote sensing. First, multiple machine learning (ML) models are trained in a cross validation context. Next, fusion is used to combat the fact that no single ML architecture is best across all data and classes. However, what have we learned? Fusion-2-text is used to discover a succinct set of important summaries or anomalies for a user in or across models.

We live in a world that is recognizing the potential of artificial intelligence (AI) and machine learning (ML) in everyday settings. These tools have been integrated 
into many aspects of our daily lives - whether we realize it or not. These tools, which were birthed from academic exercise, are no longer just in academia; they have found home in many different applications. Various AIs are being used to solve real-world problems, or they simply make our lives more convenient. Many of these algorithms are built on data-driven methods which scientists, researchers, and engineers have creatively developed and applied mathematics to build. Despite the mathematical foundations, it has become common for these tools to produce solutions that are not understandable. However, many applications require an explanation as to why a machine made a particular decision.

One task in the AI community is data or information fusion. One type of fusion revolves around the Choquet Integral (ChI), which can be learned from data. There are many ways to learn the $\mathrm{ChI}$, the reviewer can refer to 77 for a recent review. However, once these parameters are learned, explanations can be derived from the data and learned model. In [21, we exploited properties of the ChI to understand which parameters in a learned model are supported by data. In [78, we exploited this knowledge to produce indices that describe different properties of a learned ChI. Unfortunately, the large quantity of values that our indices produce can be daunting and can lead to information overload. For example, when fusing multiple deep convolutional neural networks (DCNN) for classification, there will be one ChI learned per class - a set of XAI indices for each learned ChI. As a result, there is a need to summarize these results to explain the model at a higher-level as well as reduce the amount of information that they produce.

This begs the question of how do we reduce the number of outputs while maintaining the integrity of our XAI descriptions. Herein, we explore the production of linguistic summaries that concisely describe the relevant information coming from the XAI indices. Linguistic protoform summaries (LPS) are an efficacious mechanism of describing data because natural language is easier for humans to interpret. LPSs are 
statements with a specific format that are used to describe data in natural language. LPSs have been shown to be an effective means to more easily comprehend a set of data. For example, in [79] LPSs were applied to time-series data, the authors of [80] utilized LPSs to describe the restlessness of eldercare residents for healthcare, and LPSs have also been used for data mining for knowledge discovery [81].

An LPS generally follows one of several templates. These templates fall into the category of simple protoforms or extended protoforms. The simple protoform, first introduced in [82], is constructed from three different concepts: the quantifier, summarizer, and truth value. An example of a simple protoform is "most papers are readable." In this example, the quantifier is "most," the summarizer is "readable," and the truth value would be computed to determine the degree to which that statement is valid. As time passed, others have extended the simple protoform's template [83, 79]. While there are several extensions, one example of an extended protoform (that includes an additional summarizer) is "few papers are readable and noteworthy." Not only has the LPS template been modified, but Yager's original computation of truth values has been scrutinized. In [84], it was shown that Yager's original equations to compute truth may not suited for all membership functions that model the protoforms because they may produce non intuitive summaries. As such, 84 and [85] used the Sugeno integral to overcome shortcomings in Yager's equations. Moreover, the authors of 86 present a holistic view of the development of quantifying sentences and the equations that drive this process.

The main contributions of this paper are as follows. First, we explore the potential for LPSs to reduce the complexity and amount of XAI information for the ChI. To the best of our knowledge, this has not been explored to date. Second, at a high-level, we explore what type of summaries are useful and relevant and should be reported. Third, we propose a way to derive LPSs from two of our data-centric and modelcentric indices. While this is only performed on two indices herein, due to space, 
we discuss how our procedures generalize to other XAI indices. Last, we give a case study for aggregating a set of heterogeneous architecture DCNNs for object detection and land cover classification on a benchmark remote sensing dataset. The benefit of this study is to show actual summaries and assess if they are useful.

The breakdown of this paper is as follows. In Section 4.2, we give a brief overview of the ChI and its optimization, and we identify its data supported parameters. In Section 4.3, we present the XAI indices, Section 4.4 describes how to construct LPSs, and Section 4.5 shows how to construct fuzzy sets with respect to our indices. Last, we present our case study in Section 4.6 and insights are drawn from our data and LPSs. Figure 4.1 shows the technical breakdown of our fusion-2-text and Figure 4.1 shows our remote sensing case study.

\subsection{CHOQUET INTEGRAL}

The utility of the ChI has been demonstrated in numerous applications, e.g., 24,28 , 22, 53]. The ChI is a powerful, nonlinear aggregation operator that is parameterized by a fuzzy measure (FM). Let $X=\left\{x_{1}, \ldots, x_{N}\right\}$ be a set of $N$ information sources. With respect to a finite domain, the $\mathrm{FM}, \mu: 2^{X} \rightarrow \mathbb{R}$, is a function that satisfies: (i) (boundary condition) $\mu(\emptyset)=0$, and (ii) (monotonicity) if $A, B \subseteq X$, and $A \subseteq$ $B, \mu(A) \leq \mu(B)^{1}$. It is often convenient to think about the FM as not just free parameters but as a modeling of interactions (e.g., possibly correlations) between subsets. The $\mathrm{Ch}^{2}$ is

$$
\int \mathbf{h} \circ \mu=\sum_{j=1}^{N} h_{\pi(j)}\left(\mu\left(A_{j}\right)-\mu\left(A_{j-1}\right)\right),
$$

\footnotetext{
${ }^{1}$ While not required, it is common in practice to impose $\mu(X)=1$.

${ }^{2}$ It is important to note that when a FM is set (values are specified), the ChI becomes a specific aggregation operator. For example, consider $\mu(A)=1, \forall A \in X$, except $\mu(\emptyset)$. As such, the ChI reduces to the max operator.
} 
where $\pi$ is an ordering of $\mathbf{h}=\left(h\left(x_{1}\right), \ldots, h\left(x_{N}\right)\right)^{3}$. Furthermore, $h\left(x_{i}\right)=h_{i} \in \mathbb{R}$ is the input from source $i$, such that $h_{\pi(1)} \geq h_{\pi(2)} \geq \ldots \geq h_{\pi(N)}$. Last, $A_{j}$ corresponds to the subset $\left\{x_{\pi(1)}, \ldots, x_{\pi(j)}\right\}$.

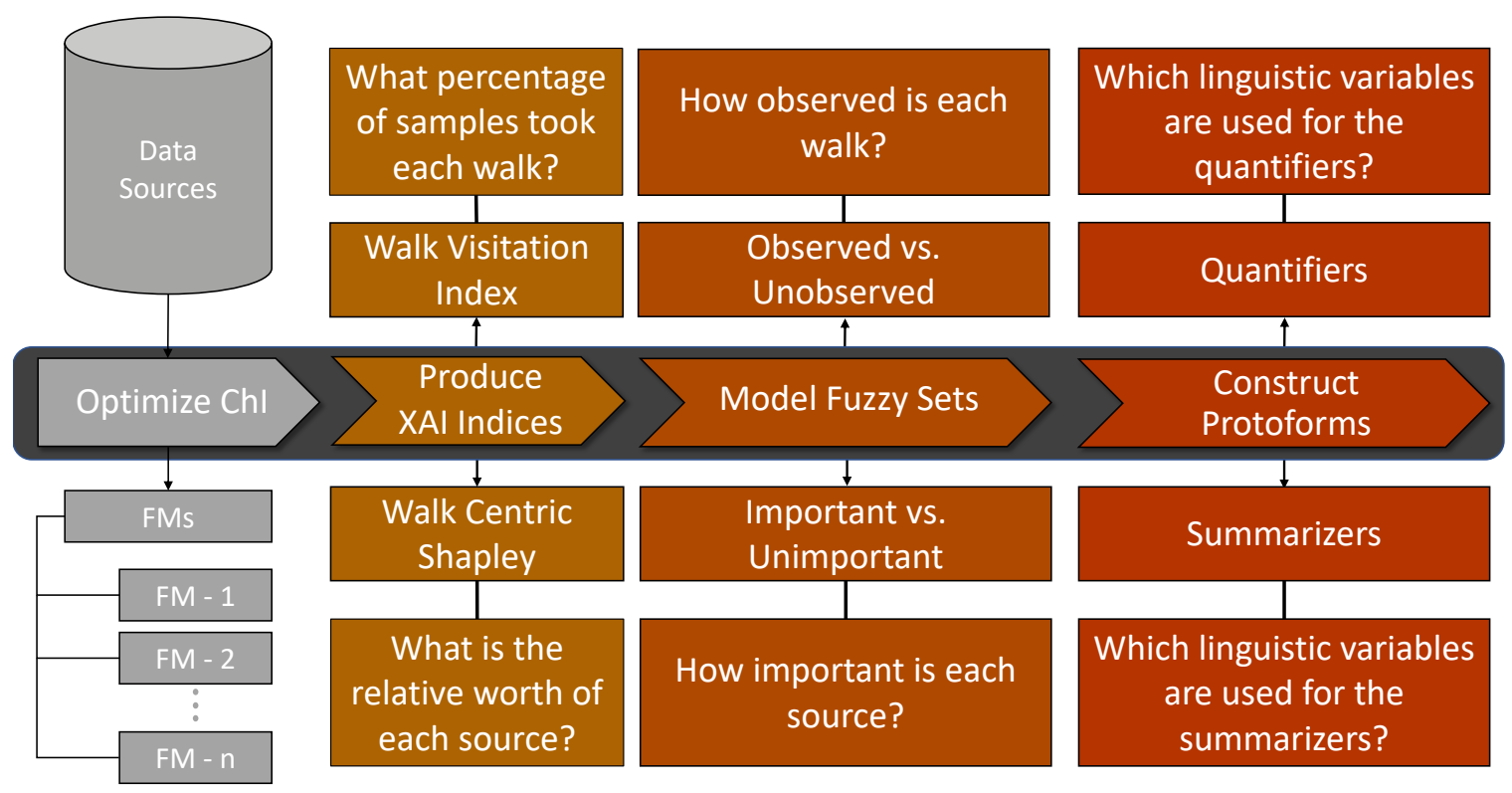

Figure 4.2: Illustration of computational stages in our fusion-2-text.

The ChI can alternatively be thought about as $N$ ! linear convex sums (LCS) ${ }^{4}$. as each sort of the data yields an LCS. Herein, we follow the nomenclature defined in [78, and we call each sort of the data a walk (in the Hasse diagram).

\subsubsection{Optimization}

Defining the FM variables in the $\mathrm{ChI}$ is not a trivial task and there are many ways to identify them, e.g., 60, 61, 63, 34]. However, in our current data-driven era, it is common place to learn the FM variables. Herein, we use our learning algorithm put forth in 21]. We do not describe the algorithm due to limited page length. The techniques proposed herein extend beyond a specific learner, they are applicable to

\footnotetext{
${ }^{3}$ Hereafter, $h\left(x_{i}\right)$ will be shortened to $h_{i}$ for simplicity.

${ }^{4}$ When $\mu(X)=1$.

${ }^{5}$ Who share $2^{N}$ weights.
} 
any $\mathrm{ChI}$ derived from data.

\subsubsection{Data Supported Variables}

In [21], we established that data supported variables can be identified for the ChI. A variable is called supported if any walk of the data includes it. For example, let $N=3$ and $h_{3}>h_{1}>h_{2}$. The FM variables that are encountered are $\mu\left(\left\{h_{3}\right\}\right)$,

$\mu\left(\left\{h_{1}, h_{3}\right\}\right)$ and $\mu(X)$. By considering all the given inputs in the training data, we can easily determine all data supported variables. This fact is important to many of the upcoming indices.

\subsection{EXISTING LOW-LEVEL XAI INDICES}

In [78], we proposed a set of XAI indices - measures/functions that summarize and highlight important properties about the FM and $\mathrm{ChI}$ - in the context of data-driven learning. In [77], we expanded our initial set of indices, including the Shapley and interaction index, to operate more accurately on partially observed domains. In general, our indices can be partitioned into three sets: information about the FM (the sources and their interactions), inquiries about the FM-ChI (e.g., what is the specific aggregation), and inquiries about the data relative to the $\mathrm{ChI}$ (e.g., what parts of our model are missing). While each of our indices provide valuable and different insights, we limit the scope herein to one data specific index, walk visitation, and one model specific index, the walk centric Shapley index.

\subsubsection{Walk Centric Shapley}

The Walk Centric Shapley (WCS) index is an extension of the Shapley index [68]. The WCS defines the relative worth of each source with respect to its data supported variables. This extension is valuable because the traditional Shapley may be drastically over- or under-estimate the worth of sources as it assumes that the FM is fully 
observable. The WC Shapley is

$$
\begin{aligned}
\bar{\Phi}_{\mu}(i) & =\sum_{K \subseteq X \backslash\{i\}} \zeta_{X, 2}(K)(\mu(K \cup\{i\})-\mu(K)), \\
\zeta_{X, 2}(K) & =\frac{(|X|-|K|-1) !|K| !}{|X| !} \mathbb{1}_{(K \cup\{i\})} \mathbb{1}_{(K)}, \\
\widetilde{\Phi}_{\mu}(i) & =\frac{\bar{\Phi}_{\mu}(i)}{\sum_{j=1}^{i} \bar{\Phi}_{\mu}(j)}
\end{aligned}
$$

where $K \subseteq X \backslash\{i\}$ denotes all proper subsets from $X$ that do not include source $i$ and $\mathbb{1}$ is an indicator function that is 1 if the FM value is data-supported and 0 otherwise.

The Shapley values of $\mu$ is the vector $\widetilde{\Phi}_{\mu}=\left[\widetilde{\Phi}_{\mu}(1), \ldots, \widetilde{\Phi}_{\mu}(N)\right]^{t}$ where $\sum_{i=1}^{N} \widetilde{\Phi}_{\mu}(i)=1$. The WCS values are important because they inform us about the relative worth of each information source.

\subsubsection{Walk Visitation}

Understanding the quality of the information sources is merely one aspect of the big XAI picture. It is also important to understand the quality (e.g., completeness) of a learned ChI. Herein, we use the walk visitation metric [78], which describes how many unique walks were taken within the training data. We quickly summarize the index due to limited page count. The index works by sorting all samples (according to their input values), finding which walks were encountered, and dividing the number of times that they were observed by the total number of samples. The goal of this metric is to determine the degree to which each walk was observed. If a probability is zero, then a walk was never seen. Furthermore, if we get a new input for the ChI and its walk was not encountered in training, then one should question the ChI output. In [78, this index was used to derive additional indices, like to what degree should we trust an output of the ChI. 


\subsection{PROTOFORMS}

Protoform-based linguistic summaries are often an effective liaison between data the data interpreter. As such, deriving linguistic summaries with respect to the XAI indices has the potential to effectively reduce the amount of information by producing concise summaries. Furthermore, as less is often more, there is also the potential to remediate confusion due to complexity, which can improve decision making. While there are multiple LPS templates to follow, the simple protoform will suffice for the insights that we are drawing herein. The simple protoform takes the following format,

$$
\text { Qy's are } P \text {. }
$$

Within the protoform, $Q$ is a linguistic quantifier, $y$ is a set of objects, and $P$ is the summarizer. Both $Q$ and $P$ are modelled by fuzzy sets over the desired domains. Examples of a quantifier that are suitable for our problem are words like "few", "many", or "most"; whereas examples of a summarizer may be "important" or "observed" when referencing the sources or the walks, respectively. Moreover, an example of a protoform with respect to the XAI indices may be "few walks are observed." With respect to the XAI indices, we produce summaries that describe the importance of each of the sources across all models and how many walks are observed in each model. To do this, we use the vocabulary in Figure 4.3 .

An LPS has a value of truth associated with it. This concept, first introduced by Yager [82, utilizes Zadeh's calculus to use Equation 4.5 to determine the truth value, $T$, associated with the linguistic summary. This equation is as follows,

$$
T(\text { Ay's are } P)=A\left(\frac{1}{N} \sum_{i=1}^{N} P\left(y_{i}\right)\right) .
$$

However, Equation 4.5 may produce non-intuitive results, as noted in 84 and then 

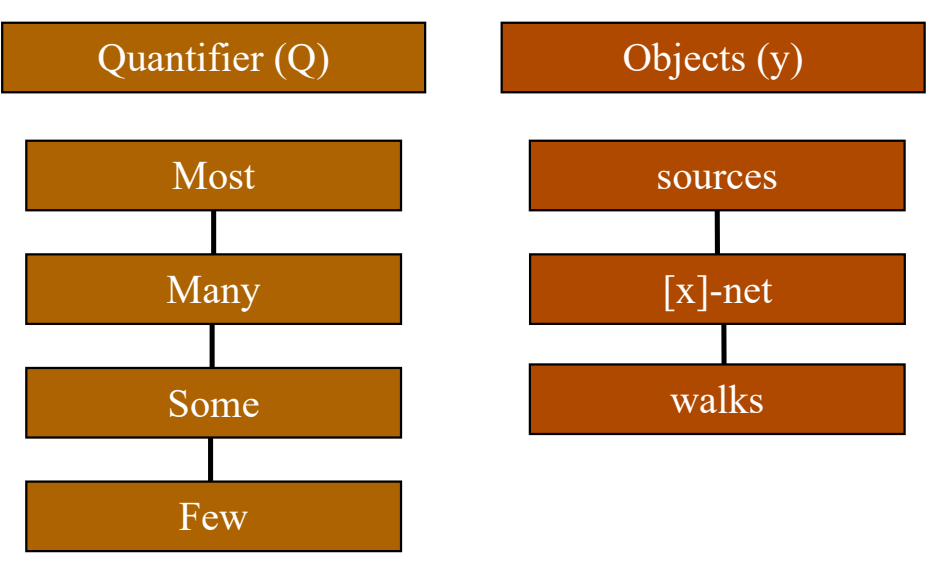

\section{Summarizer $(\mathrm{P})$}
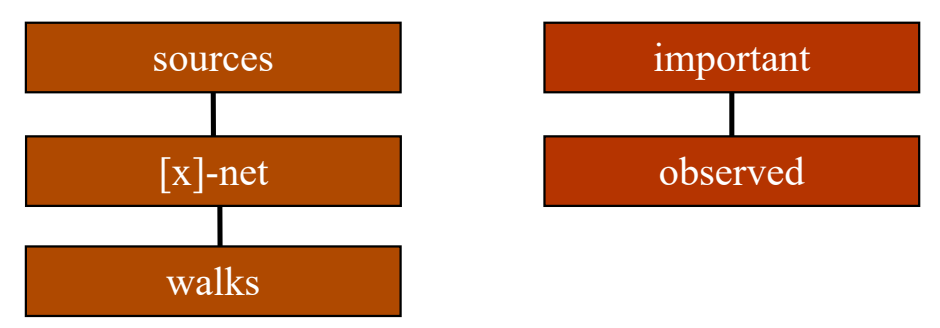

Figure 4.3: Vocabulary used herein to produce fusion LPSs.

in 85. As a result, the fuzzy integral can also be employed to determine the truth value; however, for the scope of our case study, Equation 4.5 will suffice.

\subsection{FUZZY SETS}

As mentioned in Section 4.4. $Q(x)$ and $P(x)$ are fuzzy sets. In each case, we use the trapezoidal membership function for the fuzzy sets, and we have empirically determined their parameters. This is acceptable for our initial work, as we have spent a good amount of time working with the $\mathrm{ChI}$ and the benchmark data set. However, in future work these sets and their values clearly should be learned from data or a set of experts.

\subsubsection{Walk Centric Shapley}

To define the fuzzy sets with respect to the WCS, a fuzzy set must be defined for $P(x)$ (i.e. important) and $Q(x)$ (few, some, many, most). The fuzzy set is defined by the trapezoidal membership values from the WCS values. In an ideal fusion solution, all Shapley values would be equal to $\frac{1}{N}$, meaning equal contribution. As a result, we consider the source to be important if it has a value greater than or equal to $\frac{1}{N}$. Once 


\begin{tabular}{|c|c|c|c|c|c|c|c|c|c|}
\hline Walk Visitation Quantifier & $\mathrm{a}$ & $\mathrm{b}$ & $\mathrm{c}$ & $\mathrm{d}$ & WC Shapley Quantifier & $\mathrm{a}$ & $\mathrm{b}$ & $\mathrm{c}$ & $\mathrm{d}$ \\
\hline Few & 0 & 0 & $\frac{1}{7}$ & $\frac{2}{7}$ & Few & $\frac{1}{7}$ & $\frac{1}{7}$ & $\frac{2}{7}$ & $\frac{3}{7}$ \\
\hline Some & $\frac{1}{7}$ & $\frac{2}{7}$ & $\frac{3}{7}$ & $\frac{4}{7}$ & Some & $\frac{2}{7}$ & $\frac{2.5}{7}$ & $\frac{3.5}{7}$ & $\frac{5}{7}$ \\
\hline Many & $\frac{3}{7}$ & $\frac{4}{7}$ & $\frac{5}{7}$ & $\frac{6}{7}$ & Many & $\frac{3}{7}$ & $\frac{4.5}{7}$ & $\frac{5.5}{7}$ & $\frac{6}{7}$ \\
\hline Most & $\frac{5}{7}$ & $\frac{6}{7}$ & 1 & 1 & Most & $\frac{5}{7}$ & $\frac{6}{7}$ & 1 & 1 \\
\hline Summarizers & $\mathrm{a}$ & $\mathrm{b}$ & $\mathrm{c}$ & $\mathrm{d}$ & & & & & \\
\hline Important & 0 & $\frac{1}{7}$ & 1 & 1 & & & & & \\
\hline Observed & 0 & $\frac{1}{7 !}$ & 1 & 1 & & & & & \\
\hline
\end{tabular}

Table 4.1: Trapezoidal membership function parameters.

$P(x)$ is defined, $Q(x)$ can be modelled. The output of averaging $P(x)$ is passed to $Q(x)$, and it is between $\left[\frac{1}{N}, 1\right]$ because at least 1 source will be important. As such, the domain of $Q(x)$ is $\left[\frac{1}{7}, 1\right]$. Using Equations 4.5, we can compute the truth value of each of the statements allowing us to isolate the most relevant summaries that are produced.

\subsubsection{Walk Visitation}

Similar to the fuzzy sets that govern the WCS values, fuzzy sets are used to model the walk visitation index. For $P(x)$, the fuzzy sets model the concept of observed and unobserved. In an ideal case, we desire each walk to have an equal walk visitation index, so with this in mind, we consider the walk to be observed if $z \geq \frac{1}{N !}$. Next, the fuzzy set for $Q(x)$ must be modelled. At least 1 walk will be observed, and it is possible to observe up to however many training samples exist. As such, the domain of $Q(x)$ is $\left[\frac{1}{M}, 1\right]$, which is approximately $[0,1]$. The values of each of the fuzzy sets can be found in Table 4.1. 


\subsection{CASE STUDY}

To show how these indices work in a real-world application, we consider the fusion of a set of 7 different DCNNs for object detection and land classification of remote sensing data. The DCNNs that we fuse are CaffeNet [70], GoogleNet [71], ResNet 50 [72], ResNet 101, DenseNet [73], InceptionResNetV2 [74], and Xception [75]. The dataset is the AID remote sensing data set [76]. This dataset is composed of 10,000 images over 30 different aerial scene types.

The complete training procedure of how these networks were trained can be found in [46]. Furthermore, the complete description of how these DCNNs are fused can be found in 37. Due to the nature of the multistep classification problem (DCNNs and ChI), it is an important step to determine how to split the data into training and testing. For the DCNNs, five-fold cross validation was used. This means that four folds are used for training, and one fold is used for evaluation. From the evaluation fold, two-fold cross validation is used, due to the limited number of samples in AID. To ensure that each class is approximately balanced in each of the folds, an approximately equal number of samples were chosen from each class. There are multiple ways to perform fusion across the DCNNs. Herein, we train a ChI for each of the 30 classes. As a result, there are $30 \times 7$ ! walk visitation values produced, and $30 \times 7$ Shapley values produced (a total of 151,410 values). Using the proposed LPS configuration, we reduce the XAI indices to a few sets of LPSs that are more easily comprehended.

\subsubsection{Source Summaries}

\section{XAI Question: How many sources are important?}

With respect to each ChI, we can determine how many important sources there are for each class. To produce this set of summaries, we treat each of the $30 \mathrm{ChIs}$ (one per class) as our objects, $y$. The linguistic summarizer is "important", and 
our quantifiers are "few", "several", "many", and "most". Figure 4.4 illustrates the quantity of summaries that are assigned each of the quantifiers.

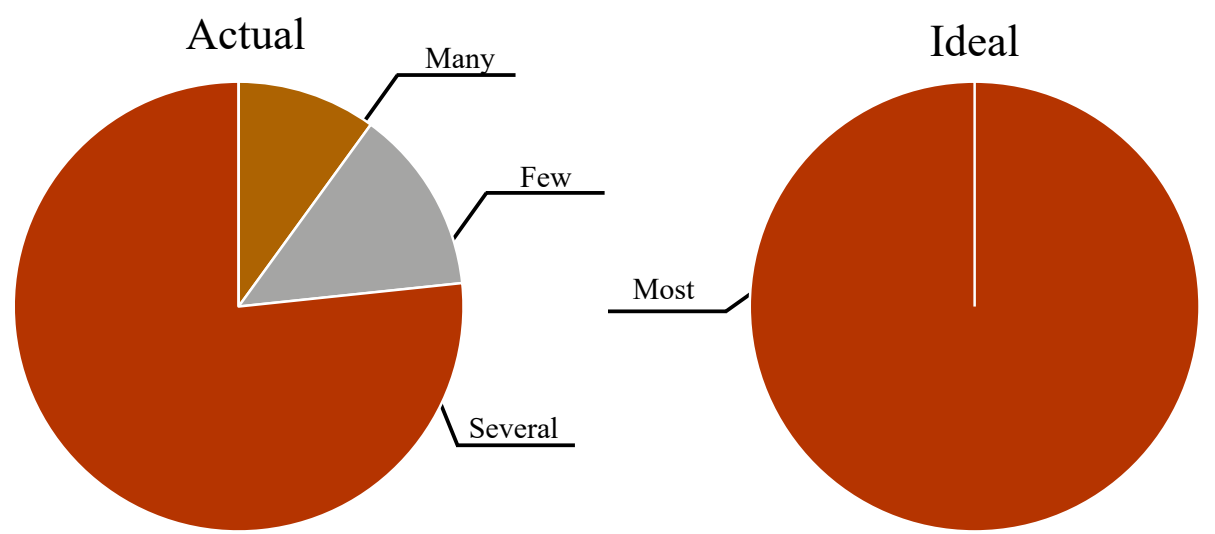

Figure 4.4: Actual versus the ideal distribution of quantifier.

In an ideal case, "most" of the sources would be important, meaning all sources are contributing to the fusion solution. However, our experiment produced no summaries of "most sources are important." The majority of the ChIs are summarized by "several sources are important." This begs the question of which sources are important because we now know that not all are important.

\section{XAI Question: How important is each source?}

Taking our knowledge from the last set of summaries, it is logical to now isolate the important sources. To do this, we produce a set of summaries specifically for each of the sources. We treat each DCNN across all models as our objects, y. For example, one set of objects will be GoogleNets, such that GoogleNets = $\left\{\right.$ GoogleNets $_{1}$, GoogleNets $_{2}, \ldots$, GoogleNets $\left._{30}\right\}$. In Figure 4.4, we show the resulting summaries.

From this set of summaries (with strong truth degrees), we can conclude that ResNet 50 and ResNet 100 are not contributing to the fusion solution; however, 


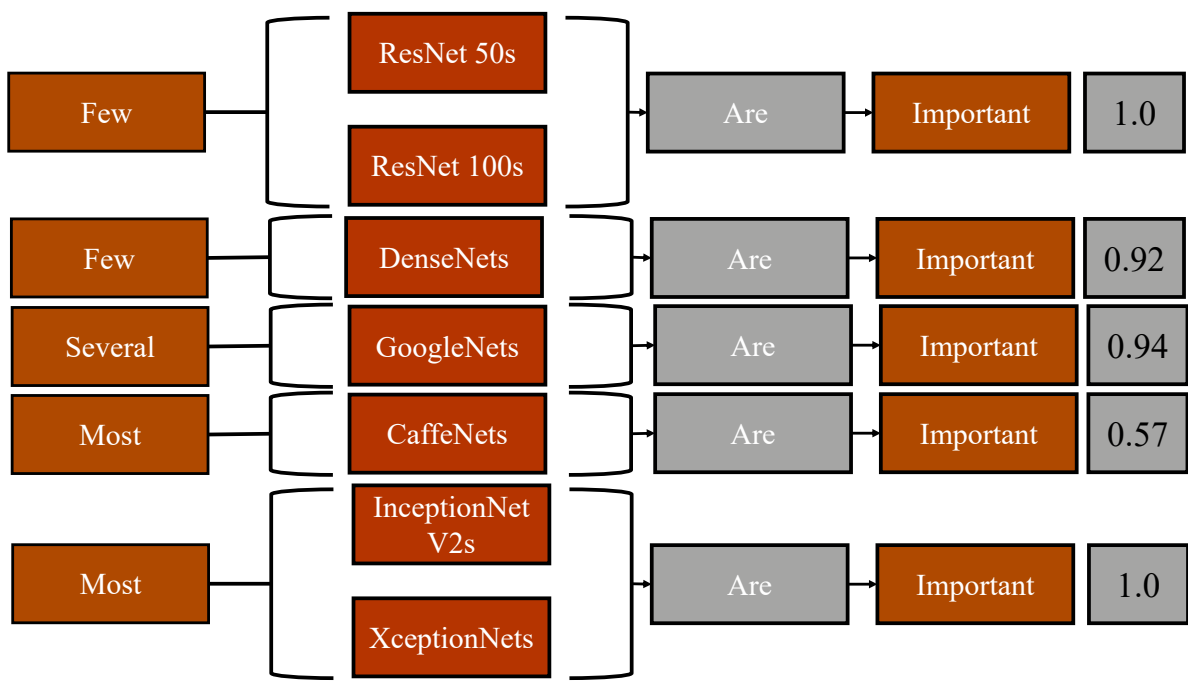

Figure 4.5: LPSs describing the importance of each DCNN with truth degree (gray).

InceptionNet and XceptionNet are important in most of the ChIs, meaning they are strong contributors. This leads us to conclude that ResNet 50 and ResNet 100 can likely be removed, speeding up inference by reducing DCNNs.

\section{XAI Question: How many walks are observed per model?}

In this case, the summarizer is "observed," the objects are each ChI, and the quantifier is again, "few", "several", "many", and "most". However, for this set, there was only one summary ever found, "few walks are observed." This quickly magnifies the flaw with these models because many of the possible walks have not been observed - meaning the FM values of many of the walks are not actually learned from data. In order to fully learn the ChI, "most" walks must be observed. Observing few walks means that there is not much diversity in the data. This highlights that we may have a dominant walk, or that we only ever observe a relatively low number of walks.

\section{XAI Question: How observed is each walk across the data?}

Whereas the last summary encapsulated information pertaining to how many 


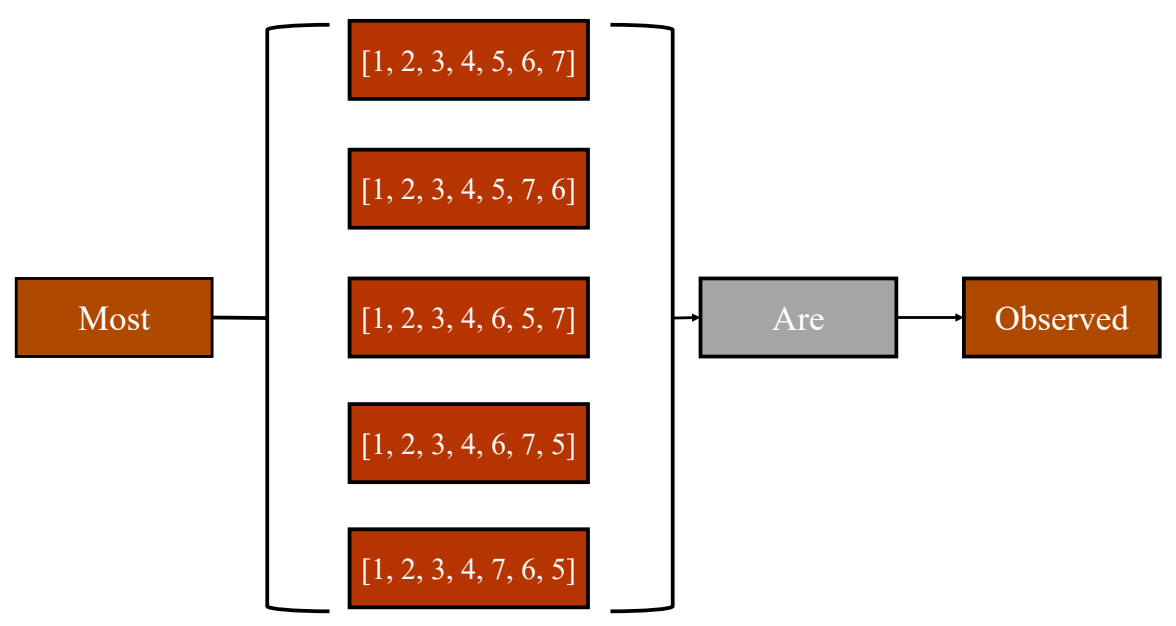

Figure 4.6: Most specific walks are observed.

walks are observed per model, this set of summaries answers the question of how observed is each walk across the entire data set. We consider each specific walk as an object; for example the walk $[1,2,3,4,5,6,7]=\left\{[1,2,3,4,5,6,7]_{1},[1,2,3,4,5,6,7]_{2}\right.$, $\left.\ldots,[1,2,3,4,5,6,7]_{30}\right\}$. When producing these summaries, there would be one summary per walk, so in our example, this would generate 7 ! summaries, which is far too many for anyone to digest. However, by only evaluating the walks that are observed in "most" ChIs with a high truth degree, we only consider 5 of the walks. The summaries shown in 4.6 are produced.

While each of these 5 walks have a truth value of 1 , they are also the only walks to have the quantifier "most". There are 4,978 walks that are observed a "few" times with a truth degree of 1 . This leaves 67 walks that are observed "few", "several", or "most" with a truth values less than 1 for each of them. These summaries clearly highlight that there may be some bias in the data. Specifically, the first 4 sources are typically encountered in the same order, which shows that something is not quite right. This allows us to dig into the data to figure out what might be going on. 


\subsubsection{Code}

The ability to reproduce an experiment is a cornerstone in the scientific community. As such, we provide the code that produced these summaries at the following repository, https://github.com/B-Mur/ChoquetIntegral.git. Moreover, the data set that was used can be found at the following repository, https://github.com/ aminb99/remote-sensing-nn-datasets.

\subsection{SUMMARY AND FUTURE WORK}

Herein, we have proposed and implemented the use of LPSs to reduce a high number of metrics to a short number of concise and more useful summaries. To our knowledge, this is the first work that produces linguistic summaries to explain fusion, without a doubt relative to data-driven learning. Before producing the summaries, the indices produce a large quantity of metrics that are complex to interpret. By producing the summaries, the indices effectively reduce the information that must be digested, while maintaining the integrity of the indices. By first determining only few sources are important, it is a logical step to determine which sources are important. If all sources were important, it would be unnecessary to determine the important sources as they are all important.

The walk visitation summaries tell a similar story. Only few walks are ever observed; this leads us to produce summaries determining the walks are observed across all data (only 5). Before we produce these summaries, these metrics are a raw stream of data that are not intuitive, and interpretable only by those with significant domain experience. However, the summaries allow someone unfamiliar with the indices (and the values they produce) to be practitioners of XAI with their fusion. To our knowledge, has never been done before.

In the future, we hope to generate summaries from the remaining XAI indices 
to provide more complete and comprehensive insights. By doing this, we will likely produce extended LPSs such that a single, extended LPS contains more information than a simple protoform can provide. We will also explore how to present, in a textual or visual fashion, this information to a human. This foundation also excites us because it is a structured format or language in which information can be extracted and then subsequently computed with. Possibilities including deriving higher-level conclusions about the data, models, and systems, or perhaps using the information to improve the training and/or use of fusion. 


\section{Chapter 5}

\section{TRANSFER LEARNING FOR THE CHOQUET INTEGRAL}

\subsection{INTRODUCTION}

A driving force behind computational intelligence (CI) is well developed mathematics. At that, many CI techniques are now learning their parameters from data. However, a caveat of learning models from data is that not all parameters may be effectively learned. Identifying ill-conditioned parameters is not always simple, as many of today's algorithms are black boxes. However, some CI methods allow for us to know which parameters are supported by data. This begs the question, how do we define unknown, or under supported, parameters.

One area within CI is information fusion. Whereas fusion is a broad topic, we limit our discussion herein to aggregation operators. Let $X=\left\{x_{1}, \ldots, x_{N}\right\}$ be $N$ sources, e.g., sensor, human, or algorithm. In general, an aggregation function is a mapping of data from $N$ sources, denoted by $h\left(x_{i}\right) \in \Re$, to data, $f\left(\mathbf{h}=\left\{h\left(x_{1}\right), \ldots, h\left(x_{N}\right)\right\}, \Theta\right) \in$ $\Re$, where $\Theta$ are the parameters of $f$. The required aggregation parameters are often too complex to be specified by a person. In such scenarios, optimization techniques exist to learn the parameters when data is present.

Herein, we explore the Choquet integral (ChI), a parametric aggregation operator. The ChI is an effective mechanism to aggregate data in many applications including explosive hazard detection [22, 23], pattern recognition [25, 27], multi-criteria decision making [28, 29], fuzzy logic [32], and deep learning for remote sensing [37]. The 
Table 5.1: Acronyms and Notation

\begin{tabular}{|c|c|}
\hline $\mathrm{ChI}$ & Choquet integral \\
\hline FM & Fuzzy measure \\
\hline TL & Transfer learning \\
\hline$X$ & Set of sources, $X=\left\{x_{1}, \ldots, x_{N}\right\}$ \\
\hline $\mathbf{h}_{j}$ & $\begin{array}{l}\mathrm{j}^{t h} \quad \text { input, } \quad \mathbf{h}_{j} \\
\left(h_{j}\left(x_{1}\right), \ldots, h_{j}\left(x_{N}\right)\right)^{t}\end{array}$ \\
\hline$\left(O^{S}, Y^{S}\right)$ & Source domain data and labels \\
\hline$\left(O_{1}^{T}, Y^{T}\right)$ & $\begin{array}{l}\text { Target domain training data } \\
\text { and labels }\end{array}$ \\
\hline$O_{2}^{T}$ & Target domain test data \\
\hline$\mu(A)$ & $\Re$-valued FM value for $A \subseteq X$ \\
\hline$\mu_{s}^{S}(A)$ & $\begin{array}{l}\text { Source domain data supported } \\
\text { variables }\end{array}$ \\
\hline$\mu_{u}^{S}(A)$ & $\begin{array}{l}\text { Source domain data unsup- } \\
\text { ported variables }\end{array}$ \\
\hline$\mu_{s}^{T}(A)$ & $\begin{array}{l}\text { Target domain data supported } \\
\text { variables }\end{array}$ \\
\hline$\mu_{u}^{T}(A)$ & $\begin{array}{l}\text { Target domain data unsup- } \\
\text { ported variables }\end{array}$ \\
\hline$E_{1}$ & Traditional ChI error function \\
\hline$E_{2}\left(\mu^{S}, \lambda\right)$ & $\begin{array}{l}\text { Regularization-based ChI error } \\
\text { function }\end{array}$ \\
\hline$E_{3}\left(\mu_{s}^{T}, \mu_{u}^{S}\right)$ & $\begin{array}{l}\text { Hard constraint-based ChI er- } \\
\text { ror function }\end{array}$ \\
\hline $\mathbf{u}$ & $\begin{array}{l}\text { Binary encoded FM vector (see } \\
\text { paper for details) }\end{array}$ \\
\hline
\end{tabular}

ChI has parameters that can be learned from data. If a parameter is encountered during training then we call it data supported. Otherwise, it is referred to as data unsupported or missing. Because missing parameters are not encountered during training, their values are left to the mercy of how the optimizer addresses them. This leaves the question of how we get missing parameters for the application at hand? Herein, we focus on how to transfer learn variables from another domain, e.g., a previously known model. 
Transfer learning is a term that refers to the learning of one model, the target, by means of another model or domain, the source. This may be necessary when there is not enough data to learn a good solution, or a good solution already exists and we want to better condition our target model. For a good review of transfer learning, see [87].The authors of [87] have identified key questions that need to be answered to have an effective transfer learning solution. By answering when, where and how to transfer, we can learn this solution. In an ideal world, transfer learning would not be necessary because there would be enough samples (without any noise) to learn the perfect solution for any machine learning (ML) task. However, CI tools have found a home in many applications, and there are not many (if any) that the perfect solution is achievable. A common task is that of categorizing visual data, and the authors of [88] have assembled a survey of transfer learning that is specific to visual categorization task. While we use this survey as an example, transfer learning methods are applicable in a variety of applications and with a variety of tools.

Herein, several contributions are made. We answer when, what and how to transfer variables specific to the ChI. Whereas it was not the focus of their article, Islam et al. actually answered then when and what in 21] by identifying supported variables in data driven learning. In Section 5.4 and 5.5, we propose two methods for how to transfer data unsupported variables from a source ChI to a target ChI.

\subsection{FUZZY MEASURE AND INTEGRAL}

The fuzzy measure $(\mathrm{FM})$ is a function $\mu: 2^{X} \rightarrow[0,1]$ with two constraints; the (boundary condition) $\mu(\emptyset)=0$ and (monotonicity) if $A, B \subseteq X$ and $A \subseteq B$, then $g(A) \leq g(B)$. The FM can be shown as a Hasse diagram, where the bottom "layer" is singletons, layer two is the tuples, an so forth. Figure 5.1 is an example for the

\footnotetext{
${ }^{1}$ Technically, $\mu: 2^{X} \rightarrow \Re_{0}^{+}$, however $[0,1]$ has advantages like property preservation, e.g., $\bigwedge_{i=1}^{N} h\left(x_{i}\right) \leq C_{\mu}(\mathbf{h}) \leq \bigvee_{i=1}^{N} h\left(x_{i}\right)$.
} 
case of $N=4$.

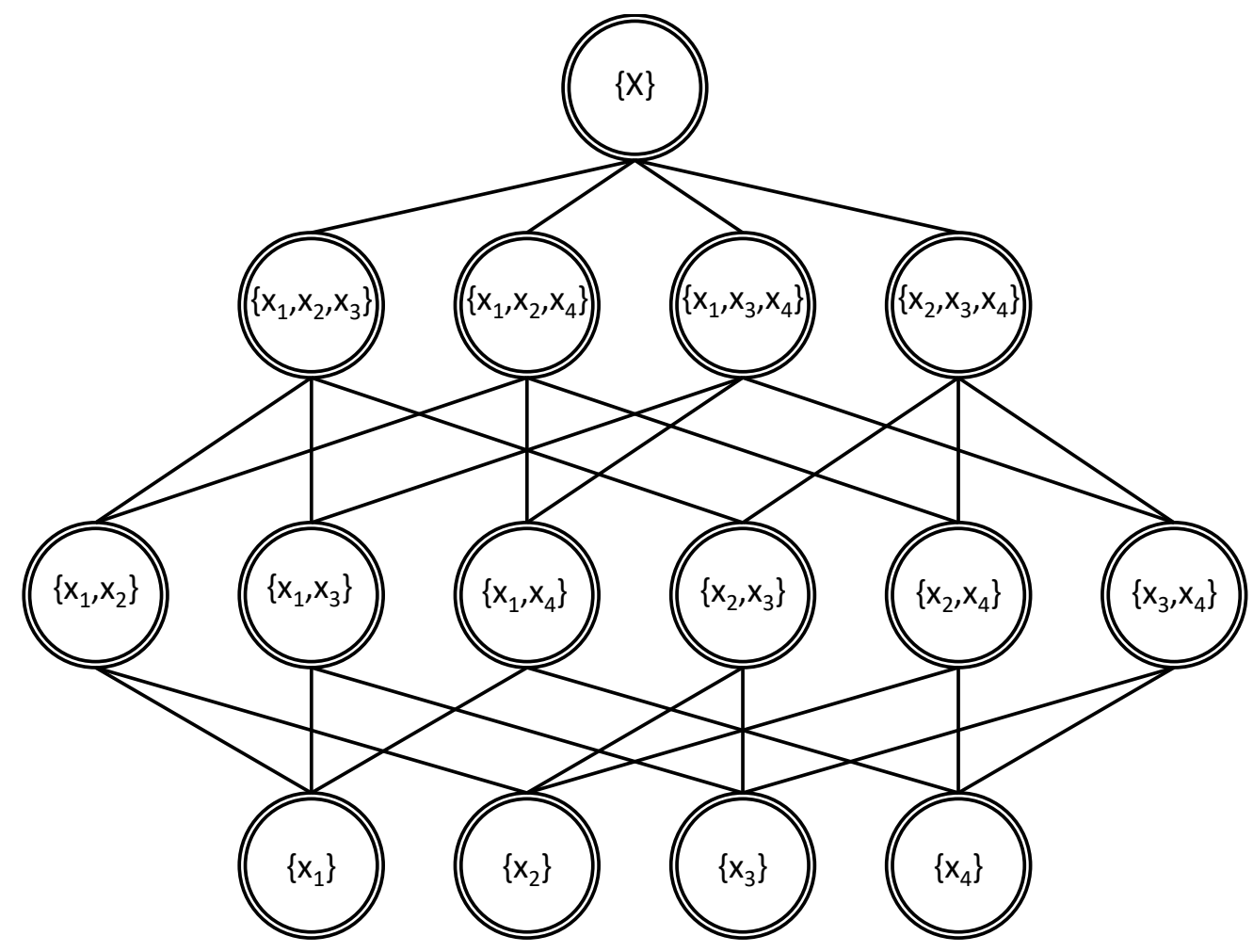

Figure 5.1: Example FM for the case of $N=4$. Circles denote subsets and edges denote monotonic inequalities.

The ChI for finite $X$ on instance $\mathbf{h}_{j}$ is

$$
C_{\mu}\left(\mathbf{h}_{j}\right)=\sum_{i=1}^{N} h_{j}\left(x_{\pi_{j}(i)}\right)\left[\mu\left(A_{i}\right)-\mu\left(A_{i-1}\right)\right]
$$

where $A_{i}=\left\{x_{\pi_{j}(1)}, \ldots, x_{\pi_{j}(i)}\right\}, A_{0}=0$, and $\pi_{j}$ such that $h_{j}\left(x_{\pi_{j}(1)}\right) \geq \ldots \geq h_{j}\left(x_{\pi_{j}(N)}\right)$. 
We can express $C_{\mu}\left(\mathbf{h}_{j}\right)$ in terms of linear algebra (see 21]), i.e.,

$$
\mathbf{u}^{t} H_{j}=\mathbf{u}^{t}\left(\begin{array}{c}
\vdots \\
h_{j}\left(x_{\pi_{j}(1)}\right)-h_{j}\left(x_{\pi_{j}(2)}\right) \\
\vdots \\
0 \\
\vdots \\
h_{j}\left(x_{\pi_{j}(N)}\right)
\end{array}\right) \text {, }
$$

where $\mathbf{u}$ is the lexicographically ordered FM, i.e.,

$$
\mathbf{u}^{t}=\left(\mu\left(\left\{x_{1}\right\}\right), \mu\left(\left\{x_{2}\right\}\right), \ldots, \mu\left(\left\{x_{1}, x_{2}\right\}\right), \ldots, \mu(X)\right)^{t}
$$

and $H_{j}$ is the size $\left(2^{N}-1\right) \times 1$ vector containing the difference of $\mathbf{h}_{j}$ terms, with respect to the $\mathbf{u}$ encoding.

As stated above, the ChI is a parametric function. As such, once the FM is specified the ChI turns into a specific operator. For example, if $\mu(A)=\mu(B)$ when $|A|=|B|, \forall A, B \subseteq X$, we obtain the familiar class of functions known as linear order statistics (LOS); e.g., the min, max, average, etc.

\subsection{DATA-DRIVEN LEARNING}

Numerous methods exist to learn the ChI, see [21]. Herein, without loss of generality, we use quadratic programming $(\mathrm{QP})$. For a set of labeled data, $O$, the sum of squared error (SSE) between the $\mathrm{ChI}$ and the known truth $\left(y_{j} \in Y\right)$ is

$$
E_{1}=\sum_{j=1}^{|O|=M}\left(H_{j}^{t} \mathbf{u}-y_{j}\right)^{2}=\mathbf{u}^{T} D \mathbf{u}+\mathbf{f}^{T} \mathbf{u}+\sum_{j=1}^{M} y_{j}^{2}
$$




$$
D=\sum_{j=1}^{M} H_{j} H_{j}^{T}, \quad \mathbf{f}=-\sum_{j=1}^{M} 2 y_{j} H_{j} .
$$

We can write the boundary and monotonicity constraints on $\mathbf{u}$ as $C \mathbf{u} \leq 0$, where

$$
C=\left(\begin{array}{c}
\Psi_{1}^{t} \\
\Psi_{2}^{t} \\
\vdots \\
\Psi_{N+1}^{t} \\
\vdots \\
\Psi_{N\left(2^{N-1}-1\right)}^{t}
\end{array}\right)
$$

and $\Psi_{1}^{t}$ is a vector representation of the monotonicity constraint, $\mu\left(\left\{x_{1}\right\}\right)-\mu\left(\left\{x_{1}, x_{2}\right\}\right) \leq$ 0 . Hence, $C$ is simply a matrix of $\{0,1,-1\}$ values of size $\left(N\left(2^{N-1}-1\right)\right) \times\left(2^{N}-1\right)$ with the form

$$
C=\left[\begin{array}{cccccccc}
1 & 0 & \cdots & -1 & 0 & \cdots & \cdots & 0 \\
1 & 0 & \cdots & 0 & -1 & \cdots & \cdots & 0 \\
\vdots & \vdots & \vdots & \vdots & \vdots & \vdots & \vdots & \vdots \\
0 & 0 & \cdots & 0 & 0 & \cdots & 1 & -1
\end{array}\right] .
$$

Herein, we solve for $\mathbf{u}$ using a QP,

$$
\min _{\mathbf{u}} 0.5 \mathbf{u}^{T} \hat{D} \mathbf{u}+\mathbf{f}^{T} \mathbf{u}, \quad C \mathbf{u} \leq \mathbf{0}, \quad(\mathbf{0}, 1)^{T} \leq \mathbf{u} \leq \mathbf{1}
$$

where $\hat{D}=2 D$.

In [21], it is shown how to identify data supported and data unsupported FM variables. The ChI starts by sorting each $\mathbf{h}_{j}$. Each index, $h_{j}\left(x_{\pi_{j}(i)}\right)$, is associated with $\left[\mu\left(A_{i}\right)-\mu\left(A_{i-1}\right)\right]$ and in total each input observes $N$ (excluding the empty set) 
FM variables, $\mu\left(A_{1}\right)$ to $\mu\left(A_{N}\right)$. Thus, one can iterate over the $M=|O|$ instances and identify FM variables not encountered in $O$. Herein, we denote data supported variables as $\mu_{s}$ and data unsupported variables as $\mu_{u}$, and their union is the full set of variables. Hereafter, we refer to $\mu_{u}$ as missing variables and the subsequent sections focus on determining how to intelligently assign values to these variables.

\subsection{TRANSFER LEARNING: REGULARIZATION-BASED}

In [89], we showed how to learn the ChI with respect to a "goal". That method minimizes an error function relative to our desire to make the answer be as close as possible to a specified FM. Whereas it was not our original intent, this procedure can be used to transfer learn the ChI.

Let $\mu^{S}$ be a source domain FM and $\mu^{T}$ be the target domain FM to learn. For example, $\mu^{S}$ could be learned on labeled source domain data $\left(O^{S}, Y^{S}\right)$. Relative to target domain data $\left(O_{1}^{T}, Y^{T}\right)$ and known FM $\mu^{S}, \mu^{T}$ can be found via

$$
E_{2}\left(\mu^{S}, \lambda\right)=\sum_{j=1}^{|O|}\left(H_{j}^{t} \mathbf{u}-y_{j}\right)^{2}+\lambda\left\|\mathbf{u}-\mathbf{u}^{S}\right\|_{2}^{2},
$$

subject to the FM boundary and monotonicity constraints, where $\lambda$ is a regularization constant, and $\mathbf{u}^{S}$ is the lexicographically encoded source domain vector (a constant). Algorithm 7 summarizes regularization-based transfer learning.

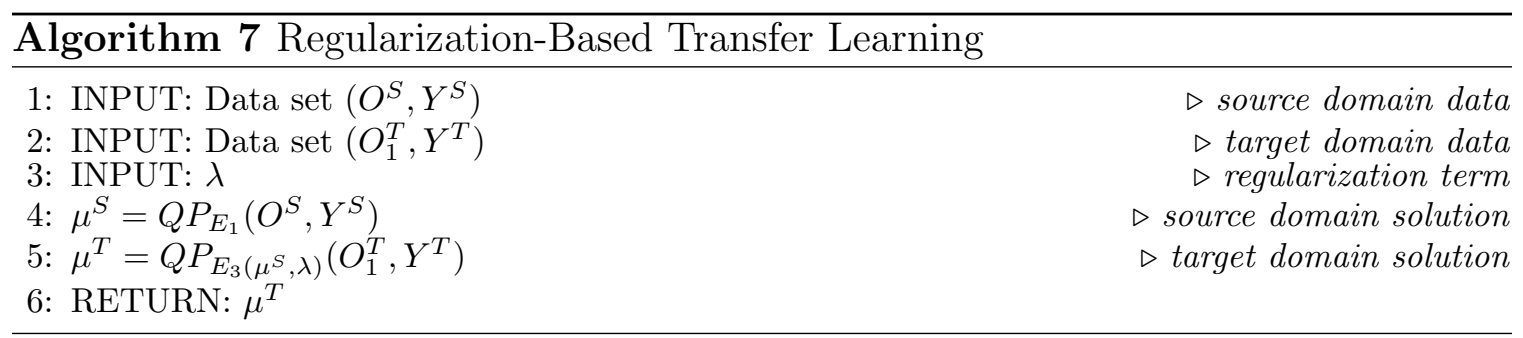

Remark 2. When $\lambda=0, E_{2}\left(\mu^{S}, \lambda\right)=E_{1}$, i.e., solve for target domain $\mu_{s}$ 's and $\mu_{u}$ 's are assigned by the optimizer. 
Remark 3. Conversely, when $\lambda$ is relatively large (with respect to the first SSE term in $E_{2}, \lambda$ drives optimization and $\left\|\mathbf{u}-\mathbf{u}^{S}\right\|_{2}^{2}$ results in $\mathbf{u}^{T}=\mathbf{u}^{S}$.

Remark 2 and 3 simply highlight extreme behavior associated with the driving factors of Equation (5.7). That is, pick too small of a $\lambda$ and get no missing variable benefit. On the other hand, picking too large of a $\lambda$ listens only to the past $\left(\mu_{s}\right.$ and $\mu_{u}$ for the source domain) and no present (target domain). It is clear that the key to Equation (5.7) is $\lambda$ selection. In this article, we use a binary grid search.

\subsection{TRANSFER LEARNING: TWO STEP OPTIMIZATION}

In this section, a second technique is explored to transfer learn the ChI (see Algorithm 8). This method is a two step optimization; learn $\mu_{s}$ 's on the target domain and then minimize the difference between the target and source domain data unsupported variables, relative to optimization ones solution.

Step one can be achieved via $E_{1}$. Next, for each $\mu_{u}^{T}$, we identify all $\mu_{s}$ 's "above" (sets of higher order cardinality) and "below" (sets of lower order cardinality). Naturally, each $\mu_{u}^{T}$ has an interval of uncertainty. Namely, it has to be greater than the larger number below and smaller than the minimum number above. Step two optimizes

$$
E_{3}\left(\mu_{s}^{T}, \mu_{u}^{S}\right)=\sum_{j=1}^{\text {missing variables }}\left(\mu_{(j)}^{T}-\mu_{(j)}^{S}\right)^{2}
$$

subject to the ChI boundary, monotonicity constraints, and the legitimate value intervals produced by $\mu_{s}^{T}:^{2}$

Remark 4. Algorithm 7 can be expressed as

$$
E_{2}\left(\mu^{S}, \lambda\right)=\underbrace{E_{1}}_{\mu_{s} \text { variables, } \mathrm{T}}+\underbrace{\lambda \sum_{\mu_{u} \text { variables, } \mathrm{T} \text { and } \mathrm{S}}^{\mu_{s} \text { set }} e_{k}^{2}}_{\mu_{s} \text { variables, } \mathrm{T} \text { and } \mathrm{S}}+\underbrace{\lambda \sum_{k}^{\mu_{u} \text { set }} e_{k}^{2}}_{k}
$$

\footnotetext{
${ }^{2}$ In $\mathrm{Eq} 5.8,(j)$ denotes indexing the missing yariables
} 


\begin{tabular}{lr}
\hline Algorithm $8 \mu_{u}$ Only-Based Transfer Learning \\
\hline 1: INPUT: Data set $\left(O^{S}, Y^{S}\right)$ & \\
2: INPUT: Data set $\left(O_{1}^{T}, Y^{T}\right)$ & $\triangleright$ source domain data \\
3: $\mu^{S}=Q P_{E_{1}}\left(O^{S}, Y^{S}\right)$ & $\triangleright$ target domain data \\
4: $\mu_{s}^{T}=Q P_{E_{1}}\left(O_{1}^{T}, Y^{T}\right)$ & source domain solution \\
5: $\mu_{u}^{T}=Q P_{E_{3}\left(\mu_{s}^{T}, \mu_{u}^{T}\right)}\left(O_{1}^{T}, Y^{S}\right)$ & $\triangleright$ data supported \\
6: RETURN: $\mu^{T}$ & $\triangleright$ data unsupported \\
\hline
\end{tabular}

where $e_{k}=u_{k}-u_{k}^{S}$.

This expansion sheds light into the inner workings of $E_{2}$. The first term only has error for data supported variables with respect to the transfer (T) domain. The second term involves data supported variables and error is defined with respect to a difference between the source $(\mathrm{S})$ and $\mathrm{T}$ domains. The third term is data unsupported variables and its error is the difference between $\mathrm{T}$ and $\mathrm{S}$.

In many contexts, $\mathrm{S}$ is past data and $\mathrm{T}$ is data for a new domain. As such, term one listens to the present, term two listens to the past and present, and term three listens to the past and present. However, these three concepts are inherently incompatible. Meaning, minimizing one causes error to rise in the other(s). On the other hand, Algorithm 8 tackles term one first, term two is ignored, and it then solves term three, but relative to the solution found in term one. The point is, Algorithms 7 and 8 are similar, but they are not the same.

Algorithm 7 could be extended,

$$
\bar{E}_{2}\left(\mu^{S}, \Lambda\right)=\underbrace{E_{1}}_{\mu_{s} \text { variables, } \mathrm{T}}+\underbrace{\Lambda_{1} \mathbf{1}^{t} \mathbf{e}_{1}^{2}}_{\mu_{s} \text { variables, } \mathrm{T} \text { and } \mathrm{S}}+\underbrace{\Lambda_{2} \mathbf{1}^{t} \mathbf{e}_{2}^{2}}_{\mu_{u} \text { variables, } \mathrm{T} \text { and } \mathrm{S}}
$$

where $\mathbf{e}_{1}^{2}$ and $\mathbf{e}_{2}^{2}$ are vectors of $\mathrm{S}$ and $\mathrm{T}$ domain differences for data supported and unsupported variables. If we let $\Lambda_{1}=0$ (i.e., do not listen to $\mathrm{S}$ domain data supported variables) then Algorithms 7 and 8 are very similar,

$$
\overline{\bar{E}}_{2}\left(\mu^{S}, \lambda\right)=\underbrace{E_{1}}_{\mu_{s} \text { variables, } \mathrm{T}}+\underbrace{\lambda \mathbf{1}^{t} \mathbf{e}_{2}^{2}}_{\mu_{u} \text { variables, T and } \mathrm{S}}
$$


Regardless, this is not the same at Algorithm 8. For example, if we make $\lambda$ relatively large, then optimization will try to make data unsupported variables on $\mathrm{T}$ and $\mathrm{S}$ be as close as possible, but at the expense of term one $\left(E_{1}\right)$. Algorithm 8 is unique because it goes the extra distance and says, do not let data unsupported variables be changed in a way that they alter what we can observe in the target domain.

\subsection{EXPERIMENTS AND RESULTS}

In this section, two synthetic experiments are presented. These experiments allow us to control which aggregation functions are learned and which variables are data supported. In order to test a range of conditions, familiar operators are selected: union-like (max and soft $\max$ ), average, and intersection-like (min and soft min). Not only do the operators for the target and source domains vary, but also the amount and placement of missing variables. For each source/target combination, experiments are performed such that only a few variables or many variables are missing from different parts of the ChI (see Figures 5.2 and 5.3 ).

The first analytic experiment emphasizes the impact of missing variables at different places in the Hasse diagram relative to different source-target domain operator combinations. The second experiment is data-driven and it compares the two transfer learning algorithms.

\subsubsection{Analytic Cases}

In this subsection, we present two examples that illustrate that the type of transfer (e.g., min to $\max$ ) and properties (cardinality) of missing variables matter. This is important because it suggests that in future work it might be possible to characterize and predict "transfer learning model error". Meaning, it might be possible to analyze attributes on the source and target domains and help the user/system decide if the estimated cost of migration is too high and transfer learning should not be used. 


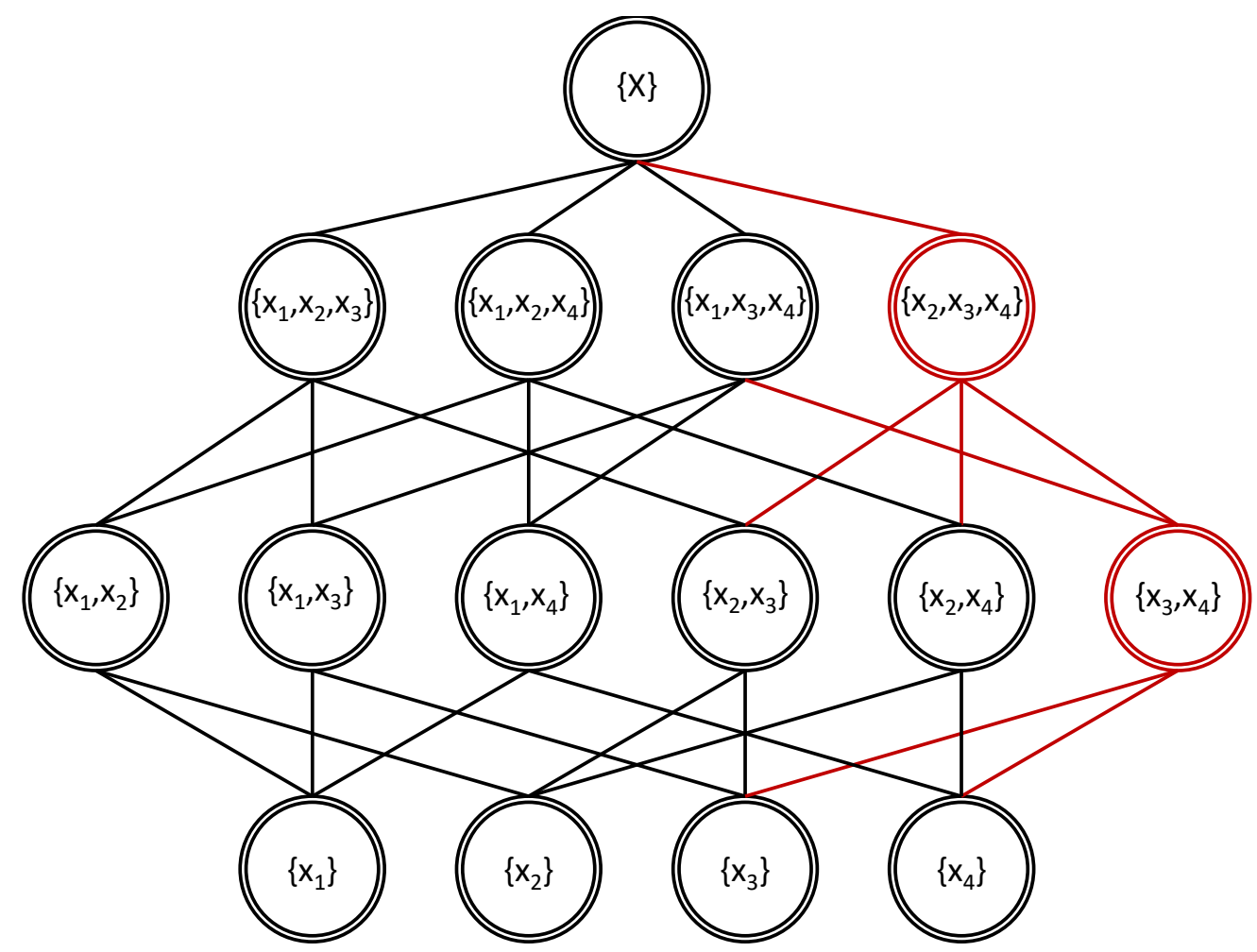

Figure 5.2: Example FM where a few variables are missing (red).

\section{Transfer t-conorm to t-norm}

Here, we explore the case of transferring a t-conorm to a t-norm (see Figure 5.4 and 5.5). First, we need to establish some notation. Each variable can be thought of as "upstream" or "downstream" relative to the other variables in the ChI. Let C denote the current variable under investigation. Upstream is all variables that include C and have greater cardinality. Downstream is all variables of lesser cardinality whose intersection with $\mathrm{C}$ is not empty.

As Figures 5.4 and 5.5 show, there is an increased risk that missing variables high in the lattice are replaced with incorrect values, versus missing variables low in the lattice. The reason is as follows. Numerically, the data supported variables are zeros and the interval of uncertainty is wide for missing variables high up in the lattice versus low in the lattice. Alternatively, high up in the lattice, upstream is one and downstream is zero. However, lower in the lattice, upstream is zero and down stream 


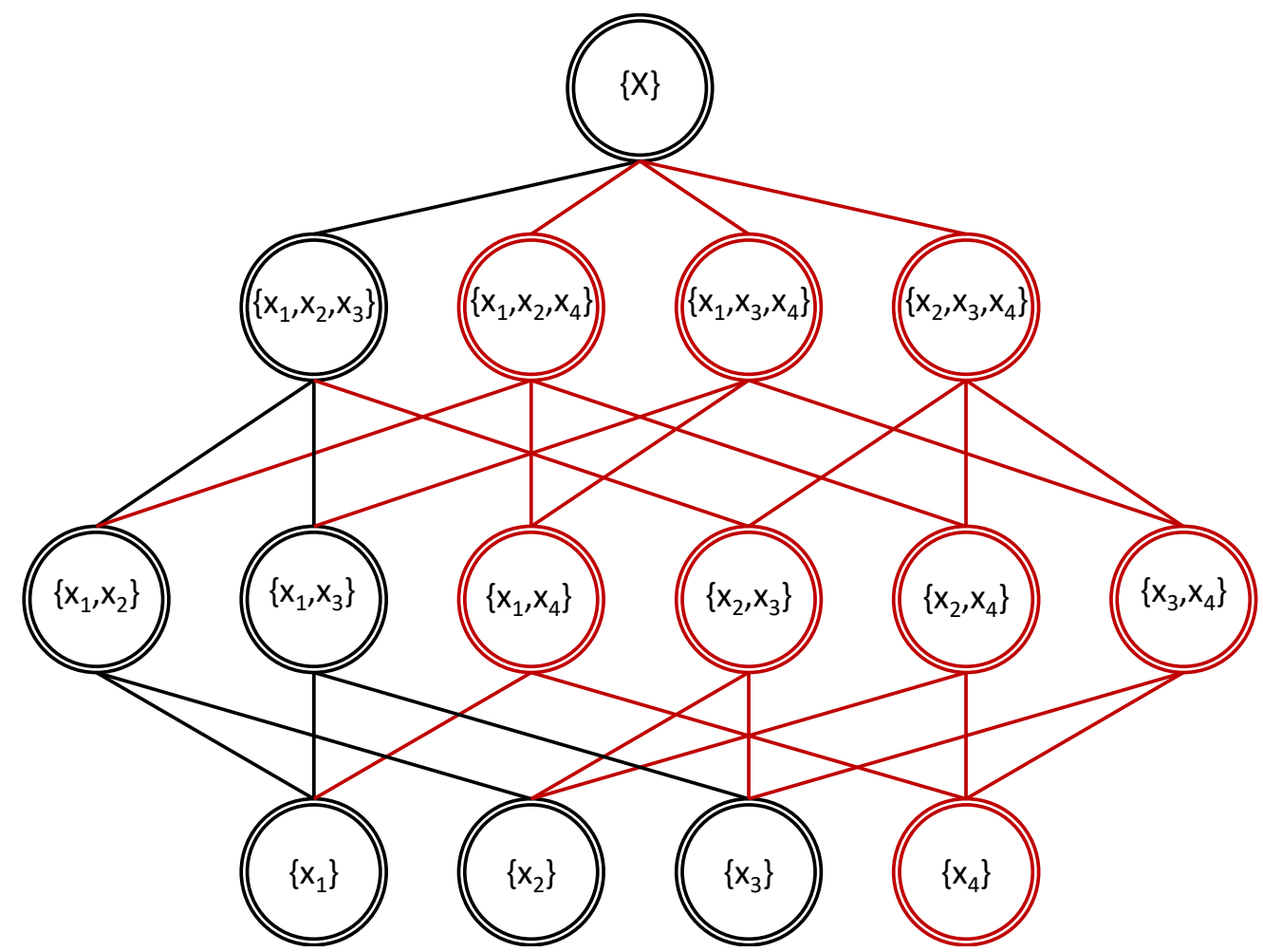

Figure 5.3: Example FM where many variables are missing (red).

is zero. As such, missing variables lower in the lattice are more-or-less "locked in". In summary, not all missing variables are created equal.

\section{Transfer t-norm to t-conorm}

In this subsection we look at the extreme opposite case of transfer learning a t-norm to a t-conorm (see Figures 5.6 and 5.7). The bottom line is, this case has the opposite effect of transferring a t-conorm to a t-norm. Namely, variables are now "locked in" higher versus lower in the lattice. In summary, missing information has a different impact on this case of transfer learning. 


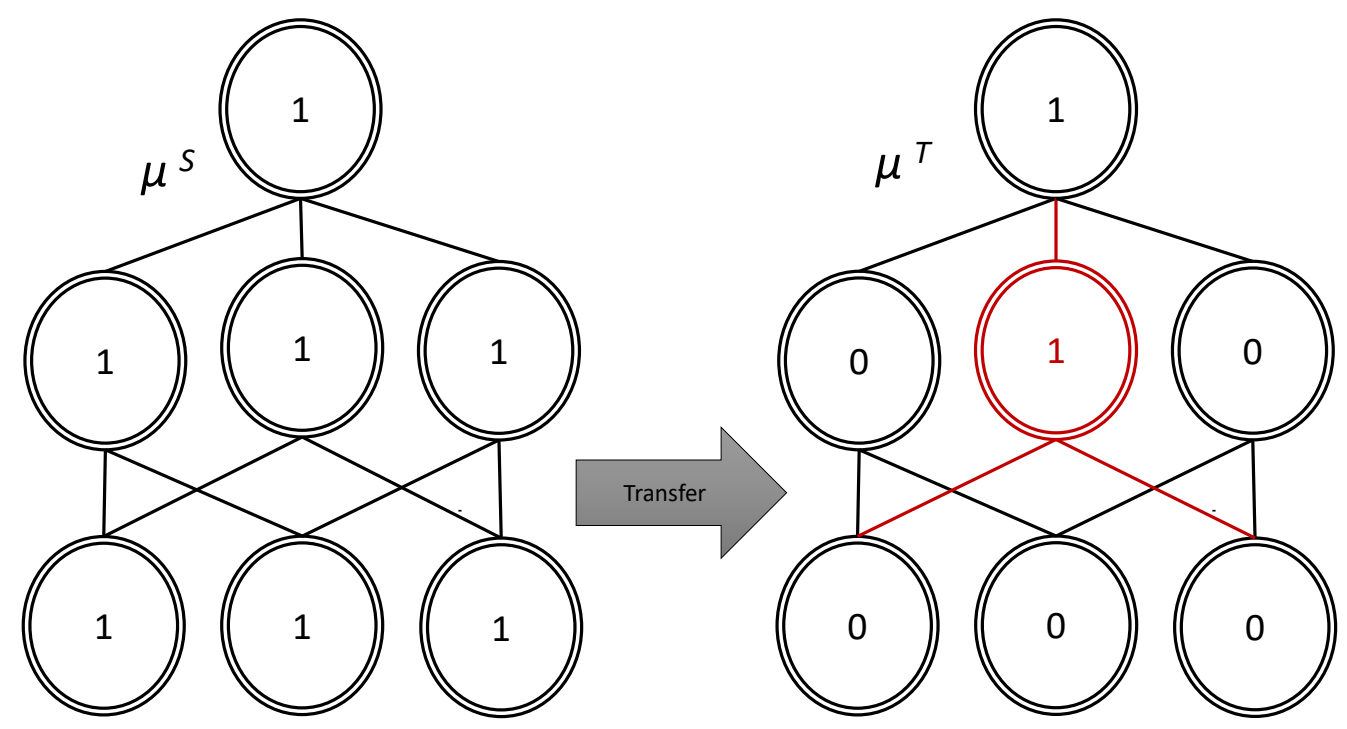

Figure 5.4: Transfer learning where $\mu^{S}$ is the maximum and $\mu^{T}$ is the minimum. The variable in red is missing towards the top of the lattice and its transfer value is zero.

\subsubsection{Regularization versus Two Step Optimization}

The following experiments compare the two transfer learning methods. To this end, the following metric is used,

$$
E_{4}\left(\mathbf{u}^{*}, \mathbf{u}^{T}\right)=\frac{1}{2^{N}-1} \sum_{i}^{2^{N}-1}\left|\mathbf{u}_{i}^{T}-\mathbf{u}_{i}^{*}\right|
$$

where $\mathbf{u}^{*}$ is the optimized FM.

For sake of tractability, we only report tables for many missing variables (see tables 5.2, 5.3, 5.4 and 5.5). The narrative is the same as few, minus exact error values. In the following experiments, $N=4$, soft max is the FM $(0.6,0.8,0.9)$, where 0.6 is the value for the singletons, 0.8 is the tuples, and 0.9 is the 3 -tuples. In addition, $\max$ is $(1,1,1)$, $\min$ is $(0,0,0)$, average is $\left(\frac{1}{4}, \frac{2}{4}, \frac{3}{4}\right)$, and soft $\min$ is $(0.1,0.3,0.6)$. We manually generated the source domain FM, i.e., it represents the ideal case of a model to transfer. On the target domain, we manually identified which variables were missing in the lattice and 500 instances were randomly generated. If a sample 


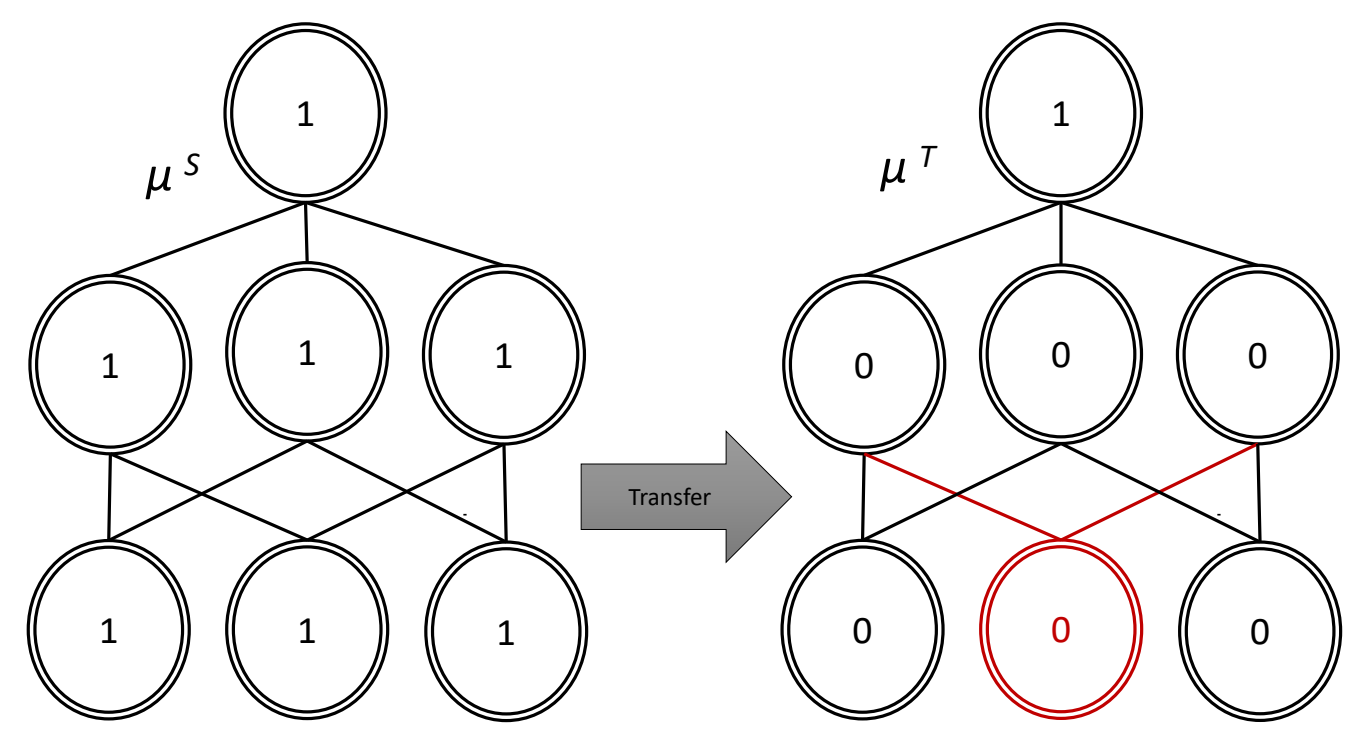

Figure 5.5: Transfer learning where $\mu^{S}$ is the maximum and $\mu^{T}$ is the minimum. The variable in red is missing towards the bottom of the lattice and its transfer value is one.

included a missing variable then it was removed. This became the training data set. For testing, a large number of instances were randomly generated, and for each sort order 50 instances were kept. This was done in order to avoid a bias in sort order, and ultimately we wanted examples that utilized all observed and missing variables.

Figures 5.8 (regularization-based) and 5.9 (two step optimization) graphically illustrate the "cost" of transfer learning (see tables 5.2, 5.3, 5.4 and 5.5). Specifically, in Figure 5.8 we see that there is no error in transfer learning an average to an average operator. However, transferring an average to a min or max has error, as we should expect. The dashed lines show the cost of transferring only a few variables. Conversely, solid lines are for many variables. As one can clearly see, the relative trends in (few, many) and (top-down, bottom-up) are intuitive; where top-down means variables missing high up in the lattice and bottom-up are missing low in the lattice. Figure 5.9 (two step optimization) has a similar story. However, the reader can clearly see that the results are not as symmetric and relative. Furthermore, the errors are higher (for this example). 


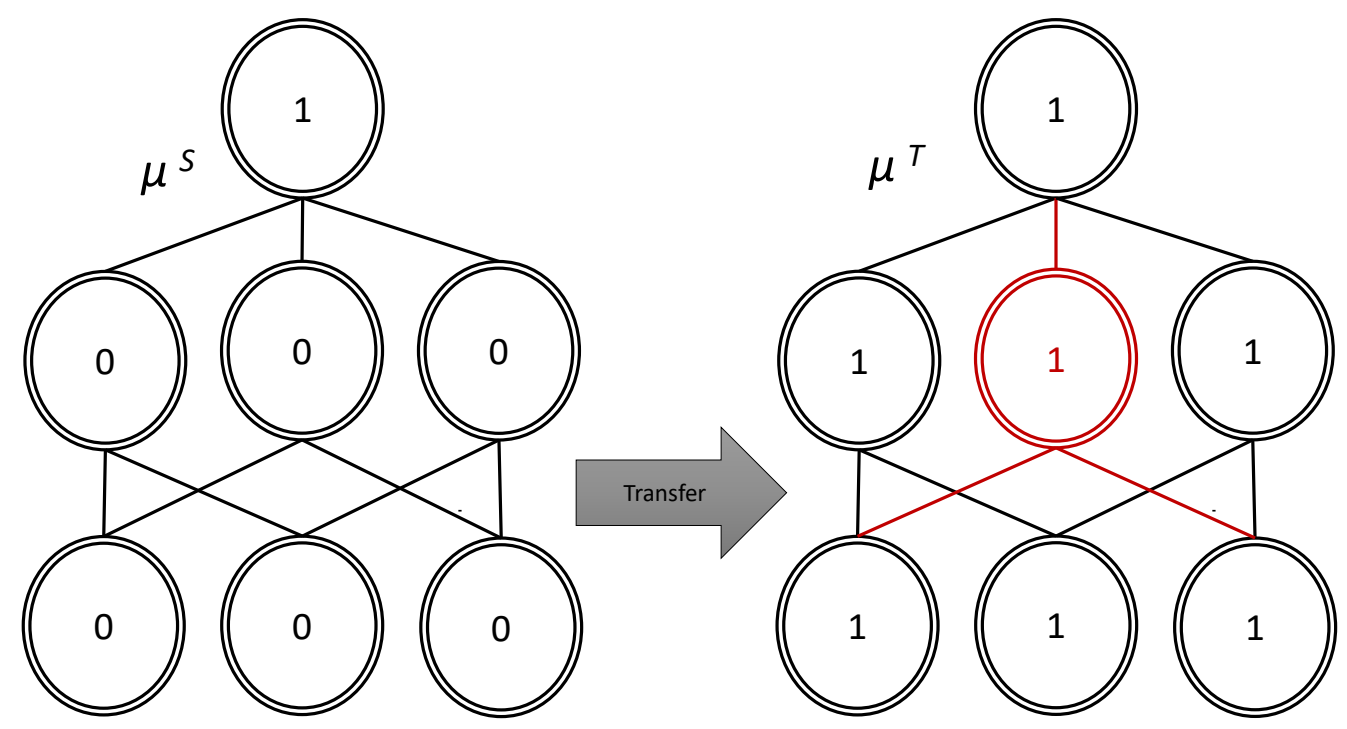

Figure 5.6: Transfer learning where $\mu^{S}$ is the minimum and $\mu^{T}$ is the maximum. The variable in red is missing towards the top of the lattice and its transfer value is one.

\begin{tabular}{|l|l|l|l|l|l|}
\hline & $\max$ & soft $\max$ & average & soft min & $\min$ \\
\hline $\max$ & 0.0000 & 0.0393 & 0.0488 & 0.0476 & 0.0491 \\
\hline soft max & 0.0692 & 0.0000 & 0.0686 & 0.0689 & 0.0695 \\
\hline average & 0.0730 & 0.0729 & 0.0000 & 0.0731 & 0.0733 \\
\hline soft min & 0.1060 & 0.1063 & 0.0727 & 0.0000 & 0.1061 \\
\hline min & 0.2638 & 0.2638 & 0.2639 & 0.2251 & 0.0000 \\
\hline
\end{tabular}

Table 5.2: Regularization Method: Many and Top-Down

We can observe in the tables a few salient trends. Namely, there is no clear winning method across the board. This makes sense to us, meaning, the regularization approach has freedom when appropriate to deviate its entire solution. On the other hand, in some cases we do not want to alter the target domain data supported variables. Our recommendation is, for supervised learning, one can evaluate both methods in a context like cross validation such that winning strategy can be selected. 


\begin{tabular}{|l|l|l|l|l|l|}
\hline & $\max$ & soft max & average & soft min & min \\
\hline $\max$ & 0.0000 & 0.0267 & 0.0500 & 0.0467 & 0.0667 \\
\hline soft max & 0.0933 & 0.0000 & 0.0900 & 0.1067 & 0.1267 \\
\hline average & 0.2166 & 0.1300 & 0.0000 & 0.0800 & 0.1500 \\
\hline soft min & 0.2538 & 0.1671 & 0.0739 & 0.0000 & 0.1751 \\
\hline min & 0.4666 & 0.3800 & 0.2667 & 0.2200 & 0.0000 \\
\hline
\end{tabular}

Table 5.3: Two Step Optimization: Many and Top-Down

\begin{tabular}{|l|l|l|l|l|l|}
\hline & $\max$ & soft $\max$ & average & soft min & $\min$ \\
\hline $\max$ & 0.0000 & 0.1377 & 0.2643 & 0.2641 & 0.2643 \\
\hline soft max & 0.1122 & 0.0000 & 0.1540 & 0.1557 & 0.1580 \\
\hline average & 0.0600 & 0.0601 & 0.0000 & 0.0601 & 0.0596 \\
\hline soft min & 0.0616 & 0.0629 & 0.0621 & 0.0000 & 0.0622 \\
\hline min & 0.0488 & 0.0490 & 0.0462 & 0.0470 & 0.0000 \\
\hline
\end{tabular}

Table 5.4: Regularization Method: Many and Bottom-Up

\begin{tabular}{|l|l|l|l|l|l|}
\hline & $\max$ & soft $\max$ & average & soft min & min \\
\hline max & 0.0000 & 0.1333 & 0.2833 & 0.3333 & 0.4666 \\
\hline soft max & 0.0933 & 0.0000 & 0.1633 & 0.2133 & 0.3466 \\
\hline average & 0.1833 & 0.1500 & 0.0000 & 0.0833 & 0.2000 \\
\hline soft min & 0.1174 & 0.1044 & 0.0633 & 0.000 & 0.1505 \\
\hline min & 0.0667 & 0.0533 & 0.0333 & 0.0400 & 0.0000 \\
\hline
\end{tabular}

Table 5.5: Two Step Optimization: Many and Bottom-Up 


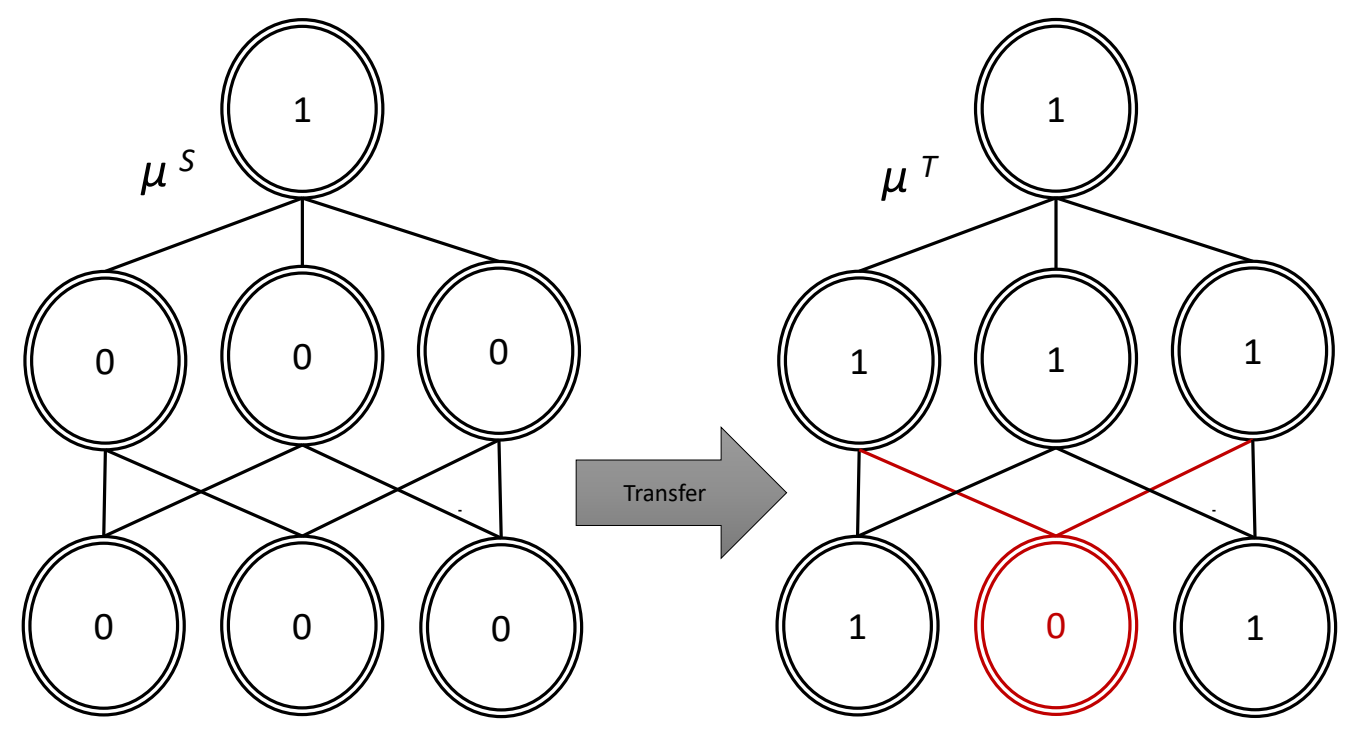

Figure 5.7: Transfer learning where $\mu^{S}$ is the minimum and $\mu^{T}$ is the maximum. The variable in red is missing towards the bottom of the lattice and its transfer value is zero.

\subsection{CONCLUSIONS AND FUTURE WORK}

Herein, we explore two ways to transfer learn the ChI. At that, we are unaware of any related efforts. To this end, we investigated our previously established regularization approach for learning with a goal. In addition, we put forth a two step optimization that sets hard limits on data supported variables relative to imputing missing variables. Our experiments and results shed light onto the inner workings of these algorithms and the fact that there is no clear winning technique.

In future work, we will apply, study, and extend this preliminary work for real applications, e.g., fusion in remote sensing. Furthermore, we will work to characterize the exact behavior of transfer learning relative to parameters like cardinality of missing variables, percentage of missing variables, and transferring between different source and target domain ChIs. An attempt will also be made to sample information from the target and source domains in an attempt to classify and help us connect estimates of predictive error relative to a user or systems need to know, "should I use 


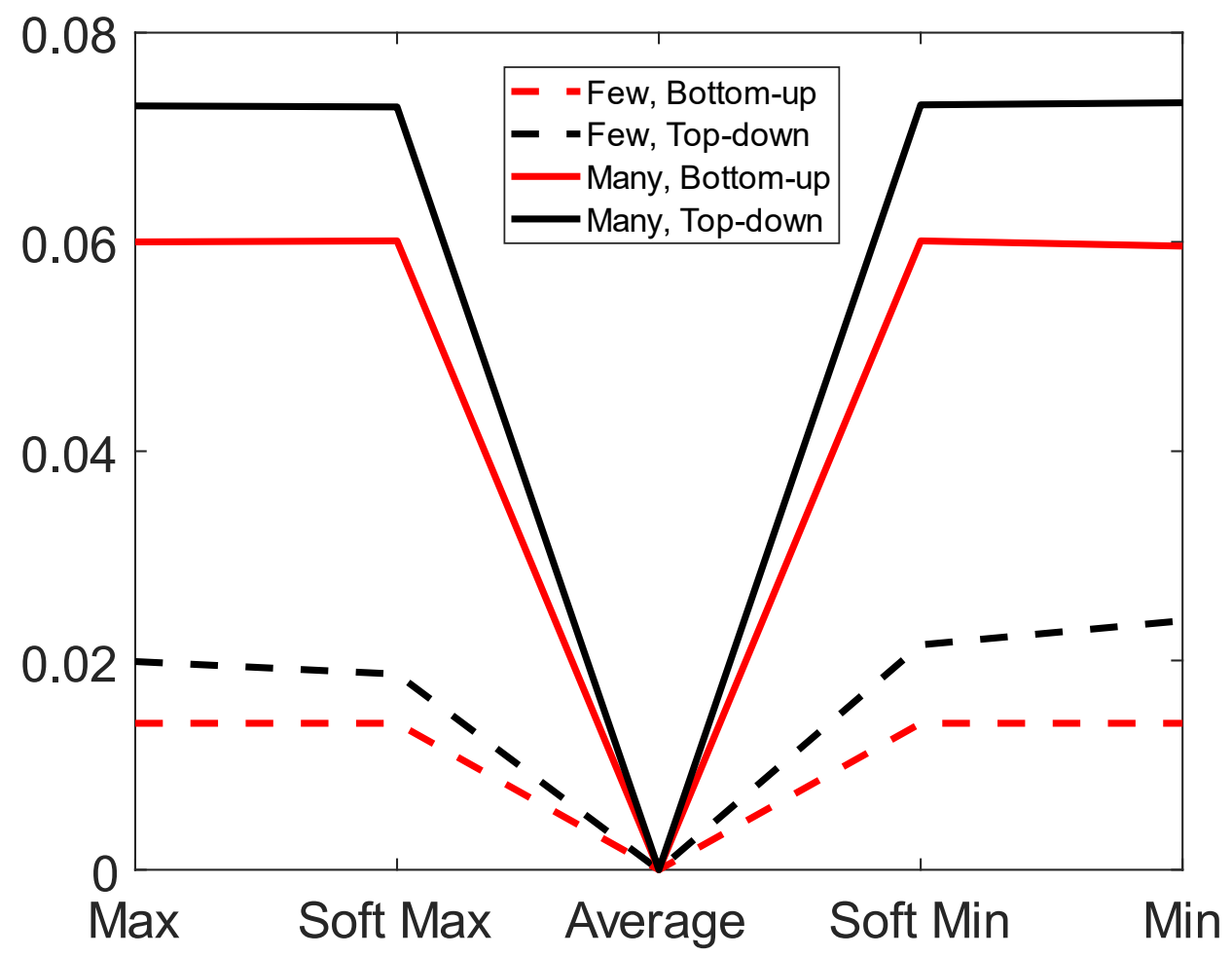

Figure 5.8: (Regularization Method) Error (y-axis) associated with transfer learning a target domain average operator relative to different source domain operators (x-axis).

transfer learning for my problem and which method should I use". 


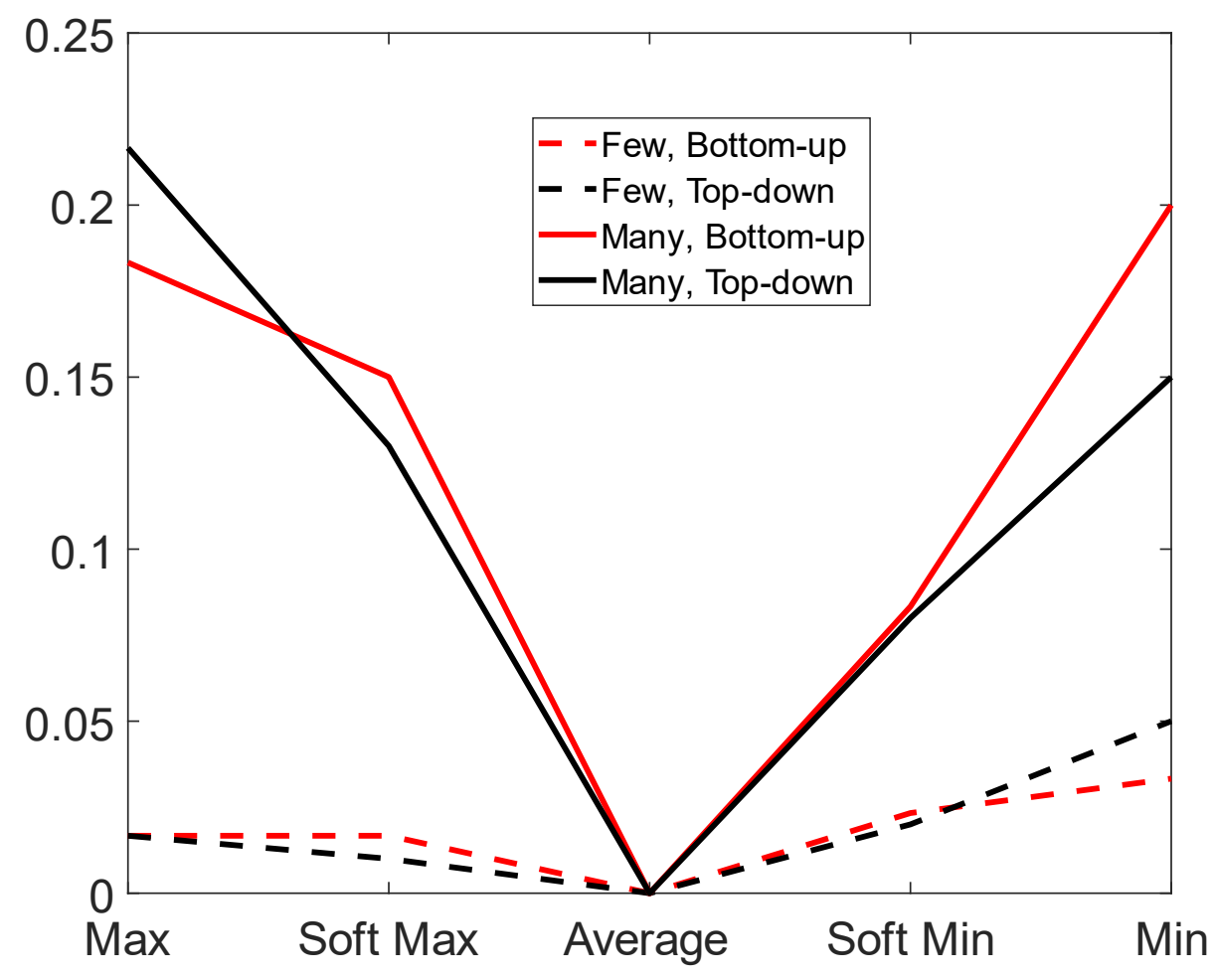

Figure 5.9: (Two Step Optimization Method) Error (y-axis) associated with transfer learning a target domain average operator relative to different source domain operators (x-axis). 


\section{Chapter 6}

\section{ACTIONABLE XAI FOR THE FUZZY INTEGRAL}

\subsection{INTRODUCTION}

Domain task-specific machine learning algorithms are gaining acceptance and are being used in real-world applications from smart cars (e.g., Tesla AutoPilot [Tesla]) to healthcare (e.g., FDA approved Siemens MRI AI Rad [Siemens]). Specialized tools include YOLO $\left[\mathbf{R e d m o n}^{\bullet} \mathbf{2 0 1 6}^{\circ} \mathbf{C V P R}\right]$ for computer vision and DeepMind's AlphaFold for protein folding [alphafold]. However, it is well-acknowledged that these algorithms are susceptible to factors like imperfections in data (uncertainty, noise, sampling, etc.), learning approach, etc. Meaning, modern AI has undesirable vulnerabilities and biases, and they are often opaque. These concerns have led to entities like the White House [white house], Defense Advanced Research Projects Agency (DARPA) [6], and industry proposing legislation and regulations concerning responsible adoption, integration, and trustworthy artificial intelligence (AI). Corporations like Google [google ai] and IBM [ibm] have even started to release open source toolkits for explainable AI (XAI).

Many high-level XAI concepts are of current interest [90], e.g., responsibility, accountability, intelligibility, etc. However, it is usually the case that we cannot precisely define them nor do we understand how to realize them. On the other hand, methods tailored to specific algorithms that produce graphical, linguistic, statistical, or other explanations are only one piece of the XAI puzzle. At the moment, XAI 
appears to be a loosely connected collection of techniques. This is not to say that "model agnostic" tools, like LIME [11, interpretability in fuzzy logic [91, or visually explaining neural networks (deconvolution [7] or Grad-CAM [92]) are not needed; it is just a statement highlighting that one algorithm is not an inclusive solution to XAI.

Herein, we focus on an underrepresented piece of the XAI puzzle, information fusion. Specifically, we focus on the fuzzy integral (FI), a computational intelligence technique used frequently in settings like aggregation, decision making, classification, pattern recognition, and beyond $[24,69]$. As we show in Section 6.3, existing FI XAI methods are relatively low-level and tailored to experts, and it is not always clear what to do with the information they return. The aim of the current article is to move beyond these individual focused works to realize a high-level actionable XAI report on which users can act. The current article is not a formal or closed system, it is a prototype coupled with exploration and discussion about directions moving forward.

The specific contributions of this article are as follows. First, in Section 6.3 we review existing methods and we categorize them according to emerging XAI nomenclature. Second, in Section 6.4 we discuss an initial set of fusion actions. Third, in Section 6.5 we discuss a fusion report prototype 11 Last, in Section 6.6, two case studies, one real and one synthetic, are provided to demonstrate action recommendations and corrections relative to tasks involving classification for multi-sensor fusion and remote sensing.

\subsection{FUZZY MEASURE AND INTEGRAL}

Let $X=\left\{x_{1}, x_{2}, \ldots, x_{N}\right\}$ be $N$ sources of information, e.g., sensors, humans, algorithms, etc., and $\mathbf{h}=\left(h_{1}, h_{2}, \ldots, h_{N}\right)^{t}$ be the input they provide $2^{2}$ The FM,

\footnotetext{
${ }^{1}$ An open source version of the software to produce this report can be found at https://github. com/B-Mur/ChoquetIntegral.git

${ }^{2}$ We use the notation $h_{i}=h\left(x_{i}\right)$ for the sake of brevity.
} 
$\mu: 2^{X} \rightarrow R^{+}$, is a function with two properties $3^{3}$ (i) (boundary condition) $\mu(\emptyset)=0$, and (ii) (monotonicity) if $A, B \subseteq X$, and $A \subseteq B$, then $\mu(A) \leq \mu(B)$. The ChI of integrand $\mathbf{h}$ on finite $X$ for FM $\mu$ is

$$
\int \mathbf{h} \circ \mu=C_{\mu}(\mathbf{h})=\sum_{j=1}^{N} w_{j} h_{\pi(j)}
$$

for $w_{j}=\mu\left(A_{\pi(j)}\right)-\mu\left(A_{\pi(j-1)}\right), A_{\pi(j)}=\left\{x_{\pi(1)}, \ldots, x_{\pi(j)}\right\}, \mu\left(A_{\pi(0)}\right)=0$, and sort $\pi$, where $h_{\pi(1)} \geq h_{\pi(2)} \ldots \geq h_{\pi(N)}$.

In total, there are $N$ ! unique sort orders and $2^{N} \mathrm{FM}$ variables. Each sort is associated with a unique walk up the FM Hasse diagram. For example, if $h_{2}>h_{1}>h_{3}$ then the ChI formula is $h_{2}\left(\mu\left(\left\{x_{2}\right\}\right)-0\right)+h_{1}\left(\mu\left(\left\{x_{1}, x_{2}\right\}-\mu\left(\left\{x_{2}\right\}\right)+h_{3}\left(\mu\left\{x_{1}, x_{2}, x_{3}\right\}-\right.\right.\right.$ $\mu\left(\left\{x_{1}, x_{2}\right\}\right)$, or $h_{2} w_{1}+h_{1} w_{2}+h_{3} w_{3}$, where $\sum_{i=1}^{3} w_{i}=1$ as $\mu(\emptyset)=0$ and $\mu(X)=1$. Thus, each walk is a linear convex sum (LCS).

\subsection{EXISTING XAI FOR THE FI}

\subsubsection{Information Indices}

Before recent acknowledgment of XAI as a formal research topic, researchers like Grabisch, Roubens, and others explored fundamental measure formulas, like the Shapley and interaction index [43], to understand the importance of individual sources and interactions among them. Since the FM has many parameters $-2^{N}$ of them-XAI requires techniques to highlight and summarize relevant information for human consumption. In [93, 94], we showed that existing methods make the assumption that a model is fully observed, which is not a reality in our data-driven machine learning era. This results in distorted answers, i.e., results computed using variables whose information are not supported by data. To remedy this, we proposed ChI

\footnotetext{
${ }^{3}$ There is an additional property that guarantees continuity for the case of infinite sets, however, in practice and in this paper, we focus on finite sets and thus this property is unnecessary.
} 
variable and walk statistics: (1) how often FM variables are observed in layer $k$, $p_{k}^{\mu}(A) \geq 0,\left(\sum_{i}^{|A|=k} p_{i}^{\mu}(A)\right)=1, A \in 2^{X} ;(2)$ observation frequency for each walk, $p^{\mu}\left(\pi_{k}\right) \geq 0,\left(\sum_{k=1}^{N !} p^{\mu}\left(\pi_{k}\right)\right)=1$; and (3) a formula to calculate what percentage of total FM variables and walks were observed. From there, we extended the Shapley and interaction indices ${ }^{4}$ to return values relative to what we know (have observed). While we showed that this improves the quality of their summarized information, these FM statistics are also a new category of FM/ChI XAI tools that can produce explanations (statistics) about the data and how those data interacted with the learned model $5^{5}$

Fig. 6.1 is a graphical depiction of two of these indices relative to fusing a set of heterogeneous deep learning architectures for object detection and land classification in remote sensing $46,37,54,6$ In summary, while indices are essential to our ability to tackle XAI, they are low-level, they require expertise, they generally are associated with information overload, and ultimately they are far from what an end user would likely consider a quality high-level human explanation.

\subsubsection{Visual Explanations}

Perhaps the most heavily used type of explanation in recent times is visual [90]. Examples for understanding convolution neural networks include guided backprop, layerwise relevance propagation, and gradient-weighted class activation mapping (GradCAM) 92 . These algorithms generally produce structures that are the same size as the input image where intensities indicate a functional relationship between pixels and network decisions, e.g., what areas in an image led to the network classifying an image as a dog. While useful, visual explanation is frequently suggestive and not a reliable source for understandability. For example, what image features versus pixels

\footnotetext{
${ }^{4}$ While we focused on the Shapley and interaction index, the underlying formula and processes can be extended to other information indices.

${ }^{5}$ In 93, 94, we produced a "truth" degree that measures how well all variables in a single ChI instance were approximated from data.

${ }^{6}$ Refer to 93,94 for full details about how to interpret Figure 6.1
} 

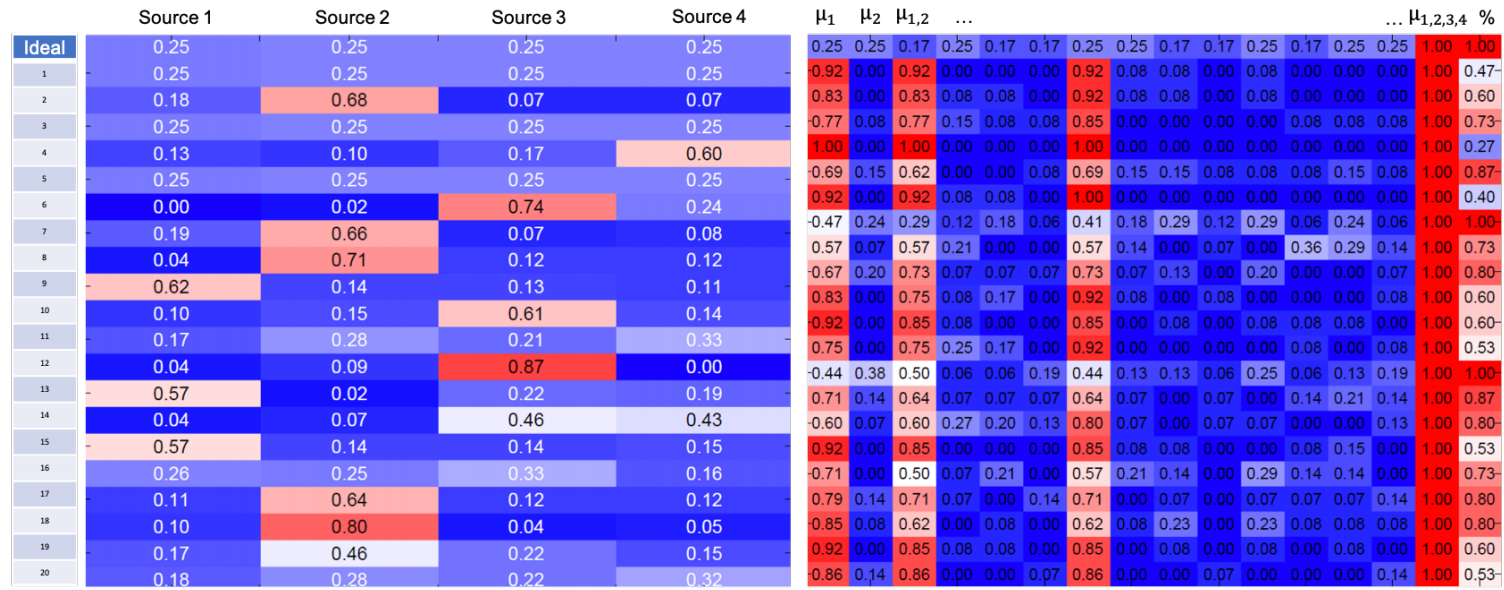

Figure 6.1: Rows are learned ChIs (one per class in the AID dataset), row one is the ideal case, and color coding goes from blue (value 0) through white (0.5) to red (1). (left) Columns are Shapley values. (right) Columns are FM variable observation frequency (lexicographical coding) and the last row is the total number of observed walks.

were used, what logic was used, etc.? It is also confusing why these algorithms return different relative results and they have deep biases (e.g., overly reliant on high frequencies) 95 .

With respect to the ChI, drastically fewer visual explanatory techniques have been proposed. In [96], we proposed a plot of the Hasse diagram relative to data sampling statistics, which informs the reader about training data completeness and which parts of an FM were approximated. In [97], we proposed a matrix visualization, which includes higher level summary information, like the Shapley and interaction index, in addition to data statistics. In [92], we proposed a way to visualize the integral and measure with respect to regularization and nonlinear regression using a non-monotonic ChI (see Fig. 6.2). Specifically, edges in the Hasse diagram are color coded by frequency of occurrence, node sizes show variable magnitudes, red and blue coloring shows positive and negative ChI regression weights, and inner circles in nodes show the effect of regularization for model simplification. In summary, these graphical explanations are an unguided exploration of the impacts of machine learning on the FI. 

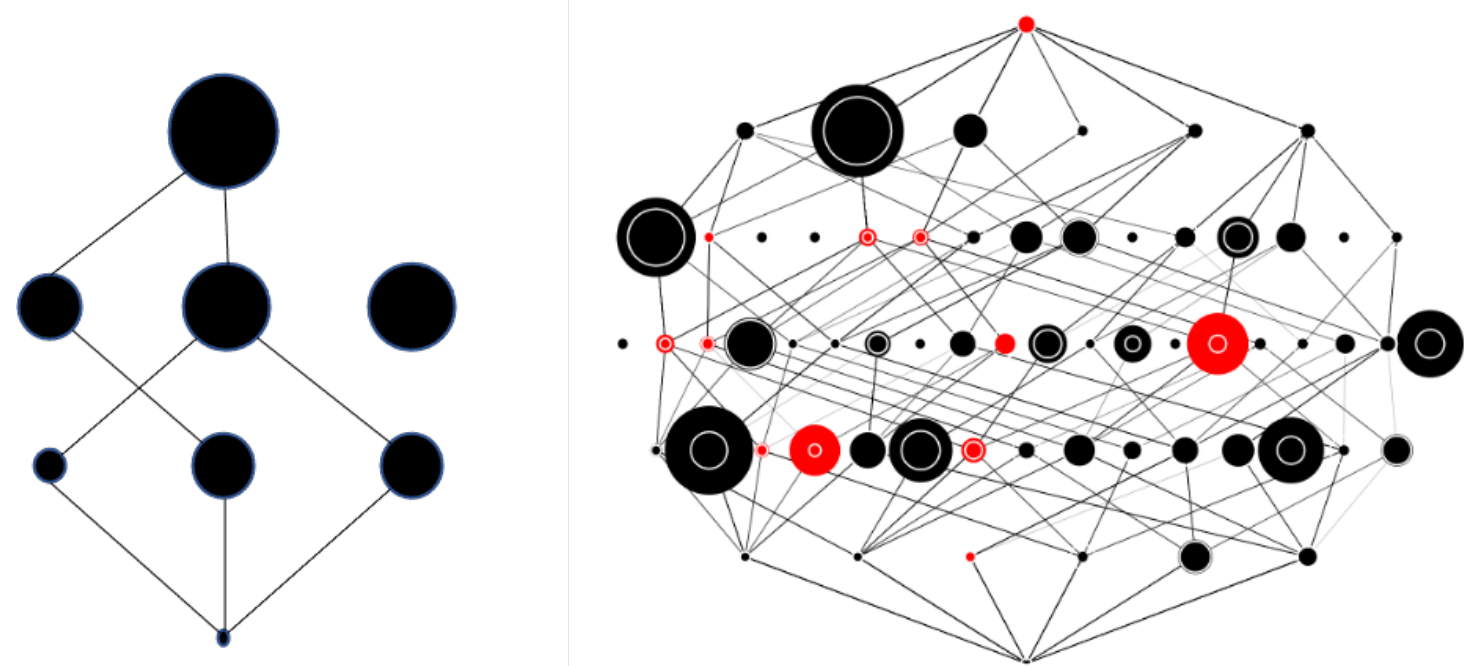

Figure 6.2: (left) Example Hasse diagram for a ChI whose training data observed three sorts, $h_{1} \geq h_{3} \geq h_{2}, h_{2} \geq h_{1} \geq h_{2}$, and $h_{3} \geq h_{2} \geq h_{1}$. The bottom is $\mu(\emptyset)$, the next layer up is $\left\{\mu_{1}, \mu_{2}, \mu_{3}\right\}$, layer two is $\left\{\mu_{1,2}, \mu_{1,3}, \mu_{2,3}\right\}$, and the top is $\mu(X)$. (right) Real diagram for the benchmark Yacht data and non-monotonic ChI for regression 98.

\subsubsection{Local Explanations}

A local explanation [90] is when a method can be decomposed into subspaces or parts for sake of understandability. In prior work [21], we highlighted that the ChI can be decomposed into $N$ ! different LCS subspaces, each of which can be interpreted and then explained to a user. Without loss of generality, consider the input sort $h_{2}>h_{1}>h_{3}$ and an arbitrarily selected set of corresponding difference in FM weight: 7 , $\mathbf{w}=(0,0,1)^{t}$. The underlying associated logic is,

If input 2 is greater than $1\left(h_{2} \geq h_{1}\right)$, and input 1 is greater than 3 $\left(h_{1} \geq h_{3}\right)$, then take a pessimistic stance, select the value they all agree with, i.e., $h_{3}$.

Specifically, we disregard sources 2 and $1\left(w_{1}=w_{2}=0\right)$ and listen fully to source $3\left(w_{3}=1\right)$. This local explanation is possible because the ChI can be decomposed

\footnotetext{
${ }^{7}$ Where $w_{i}=\left(\mu\left(A_{\pi(i)}\right)-\mu\left(A_{\pi(i-1)}\right)\right)$.
} 

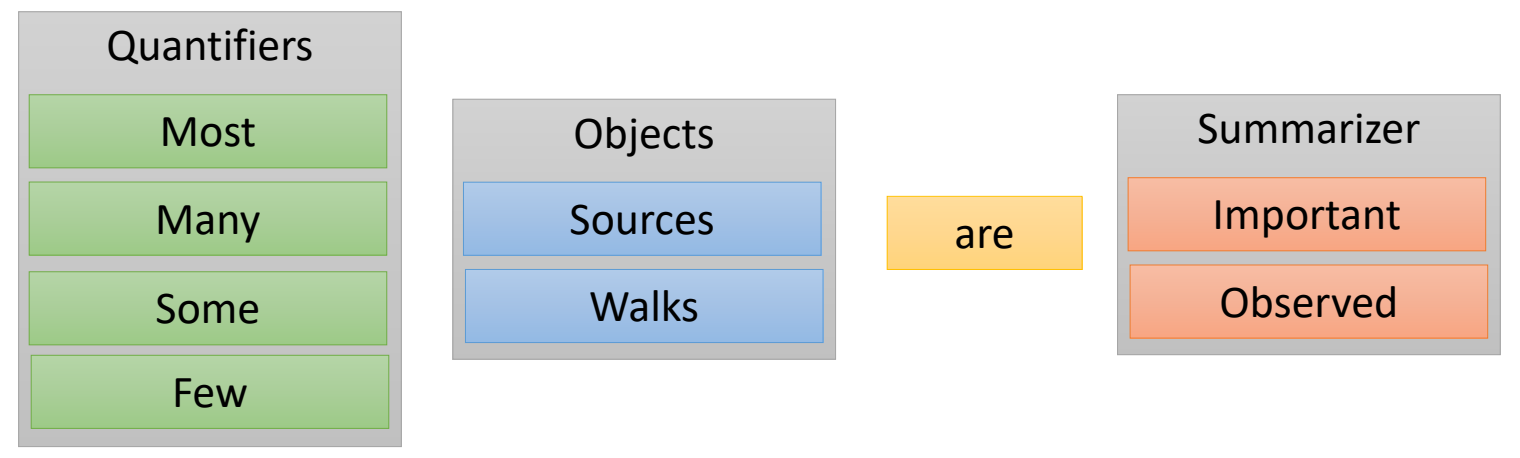

Figure 6.3: Subset of the vocabulary for generating linguistic protoform summaries for XAI for the ChI. Introduced in [99]

into a set of LCSs, and each LCS can be interpreted via its weights. The process is completely transparent when the inputs are understandable, as occurs in many human-centric multi-criteria decision making applications [69]. However, in the case of our prior work on fusing a set of heterogeneous deep learning networks [46, 37, 54, understandability is hindered by the opaqueness of the inputs, i.e., the deep neural network outputs.

\subsubsection{Text or Linguistic Explanations}

An important concept in fuzzy set theory is linguistic summarization. Many domains are plagued with big data and information overload. Linguistic summarization is the task of distilling important trends in data and translating them into succinct linguistic statements for human consumption. In [99], we generated fusion linguistic protoform summaries (LPS) of the form " $\mathrm{Q}$ y's are $\mathrm{P}$ ", where $\mathrm{Q}$ is the quantifier, $\mathrm{y}$ is the object, and $\mathrm{P}$ is the summarizer. Fig. 6.3 shows the LPS fusion vocabulary used in [99].

In [46, 37, 54, we fused 7 different deep learning classifiers. The benchmark dataset had 30 different classes so there are $30 \times 7$ ! walks and $30 \times 7$ Shapley values, that is, 151, 410 raw measurements. In return, there were only 42 LPSs with a high truth value, e.g., 
P1: "Most Inception Net Models are Important,"

P2: "Few ResNet Models are Important,"

P3: "Many $\pi=(2,1,3)$ Sorts are Observed."

Summaries can be produced with respect to a single trained ChI to understand a single model, or across a set of trained ChIs - one per class or across folds in cross validation - to discover trends. Note, these summaries rely on lower-level XAI tools like the indices discussed in Section 6.3.1. To date, these LPSs are the only high-level form of automatic FI explaination that we are aware of.

\subsection{ACTIONABLE XAI AND THE FI}

The explanations outlined above have been used to provide insight into the past, namely a trained model and the ChI output. While this helps us address the question, on what basis was a decision made?, it is still a massive amount of information (indices, local, visual, linguistic). And what do we use them for? This question is a deeper XAI inquiry and the focus of the remainder of this paper. As the FI is used for numerous tasks, like decision making, classification, regression, morphology, and beyond, it is not possible to imagine all possible applications and actions therein. The approach we take is to study fundamental actions, referred to hereafter as atomic activities to denote that they cannot be further decomposed. The three activities highlighted herein are a starting point that help us ameliorate common challenges.

\subsubsection{Atomic Activity 1: Acquire New Data}

Let $T=(\mathbf{y}, H)$ be a set of $M$ training examples, where $\mathbf{y}=\left(y_{1}, \ldots, y_{M}\right)^{t}$ are the training labels and $H=\left\{\mathbf{h}_{1}, \ldots, \mathbf{h}_{M}\right\}$ are the input feature vectors. Modern machine learning is based on sampled data and model fitting. In the case of an AI algorithm

like a neural net, which are generally distributed and implicit models, it is not clear 
which parameters have been approximated to what degree from the observed sample set. However, the ChI is a centralized and explicit model. As highlighted above, each $\mathbf{h}_{i}$ has a sort, $\pi_{i}$, and therefore a fixed set of associated FM variables. As a result, we can easily produce a probability distribution over the set of all possible walks, $p\left(\pi_{k}\right) \geq 0,\left(\sum_{k=1}^{N !} p\left(\pi_{k}\right)\right)=1$, where $p\left(\pi_{k}\right)=\frac{1}{M}$ is the ideal uniformly distributed case. A user can decide what constitutes a problematic walk, e.g., $p\left(\pi_{k}\right)=0$ or $p\left(\pi_{k}\right)<\tau$. Therefore, it is possible to convert $T$ into a request for a specific set of contexts that need to be completed to complete the model. For example, consider a problem from multi-sensor fusion where sources are sensors. The atomic activity, acquire new data, would result in a request like,

Collect new training data where sensor 2 has the highest return, followed by sensor 3 then 1;

Collect new training data where expert 1 is the most confident, followed by expert 3 then 2.

Granted, data collection can be expensive (money, time, etc.). While these suggestions are easy to generate, the burden ultimately rests on the application domain.

\subsubsection{Atomic Activity 2: Remove Source(s)}

The next atomic action is remove a source, i.e., $\hat{X}=X \backslash x_{k}$. Computationally, this is of little benefit as the ChI is merely $N$ multiplications and $N-1$ additions. Storage wise, eliminating sources can greatly lessen the storage and complexity demand of the $2^{N}$ variables and $\left(N\left(2^{N-1}-1\right)\right) \times\left(2^{N}-1\right)$ monotonicity constraints. Perhaps more convincing reasons for removing sources are the physical cost per source (e.g., expensive sensor, invasive medical procedure, etc.), desire to scale to larger $N$ (which is otherwise prohibitive due to the number of variables and constraints), and machine learning benefits of reducing redundant and irrelevant features that plague 
many optimization algorithms. While it is one thing to decide to remove a source, it is another to remove it. Here, we highlight two options. Option one favors machine learning; we simply remove the source(s) and retrain the integral. A second option was discussed in 36 for missing data. Two techniques were provided for removing FM variables associated with the removed source(s), relative to adhering to the monotonicity, boundary conditions and underlying semantics.

\subsubsection{Atomic Activity 3: Add Source}

The last action discussed here is add a source, $\hat{X}=X \cup x_{N+1}$. Unlike action 2, this increases the computational, storage, and associated costs with the aim of improving a task specific goal such as accuracy or F1 score for regression, classification, or decision making. This action comes with the caveat of introducing new parameters in the ChI. As in Section 6.4.2, one option, when/where applicable, is to add the new source and retrain the integral. However, this action comes with the complexity of access to the new source and associated training data. A second option is to add new FM variables, but the question is, where do we obtain these values and how do we augment an existing model? While the prior is straight forward, to the best of our knowledge the latter is unsolved (and remains for a future work).

\subsection{XAI FUSION REPORT}

Next, we combine the methods in Sections 6.3 and 6.4 to generate an XAI fusion report; see Fig. 6.4). Our aim is to convey relevant visual, linguistic, statistical, and associated information in the context of recommending actions. The goal is a user friendly report that can be used by FI novices.

In Fig. 6.4, the left column has the top selection of rank-ordered recommended actions along with any associated caveats and disclaimers.8.$^{8}$ Each of these actions are

\footnotetext{
${ }^{8}$ Caveats are predetermined text recommendations that surround and support the highlighted actions. Disclaimers provide context, they generally impact multiple actions, and they tend to be
} 


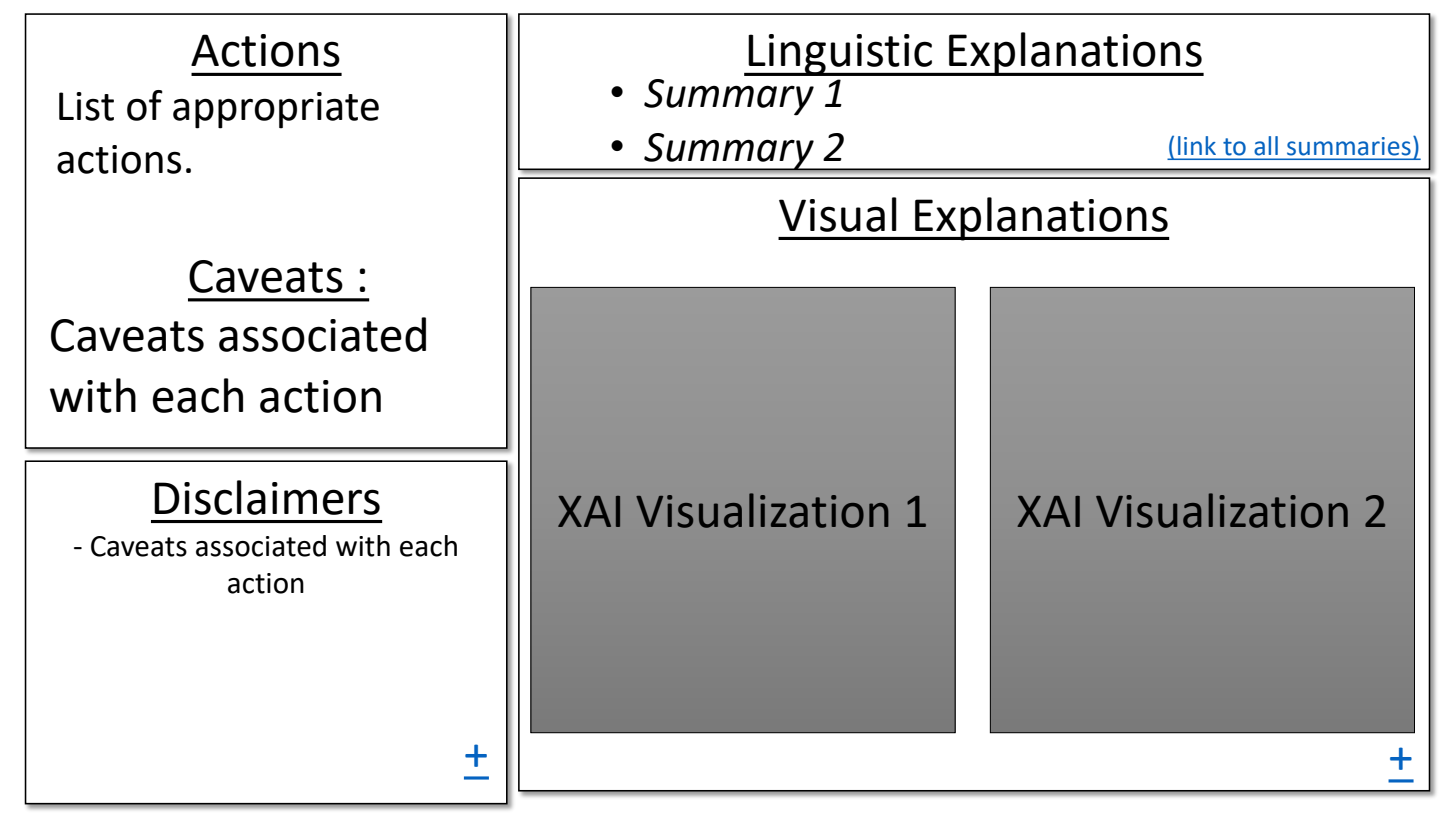

Figure 6.4: Actionable XAI fusion report template. The left column has context, actions, and assistance. The right column has low-level XAI details, e.g., linguistic, graphical, etc. Each box has has a link to expand if a user desires access to the full set of underlying explanations.

supported by a set of underlying statistical, linguistic, and visual explanations, aka, the right column boxes. Our idea is to initially report a minimal, yet informative, amount of information as to not overburden the user. However, each box has an expansion icon that opens up the full set of associated data. No information is ever lost, it is just prioritized for human consumption. This allows the interface to be used as a tool for analyzing the decision making chain in addition to action recommendation. The following two case studies highlight the fusion report.

vague. For example, the user might want to retrain if the learning method is non-deterministic, seek a new learning algorithm, etc. 


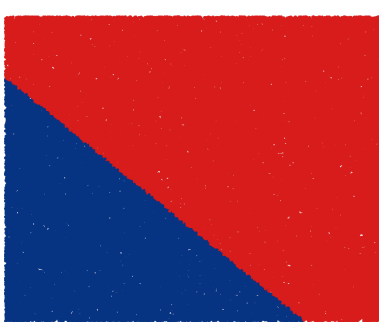

Source 1

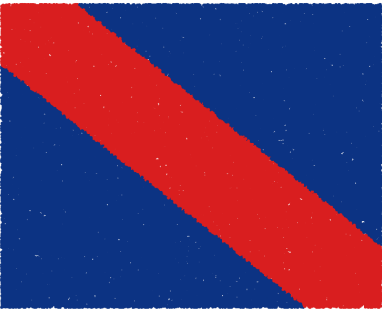

Ground Truth

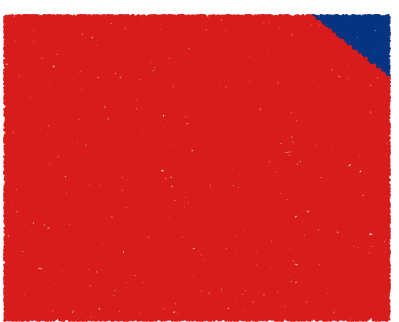

Source 2

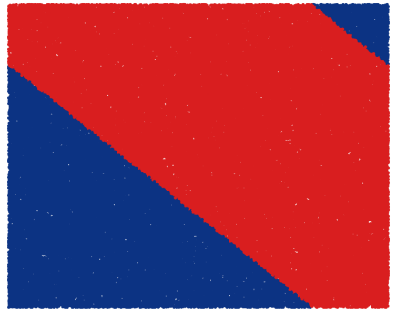

Source $1+$ Source 2

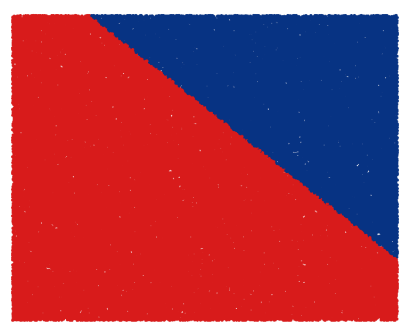

Source 3

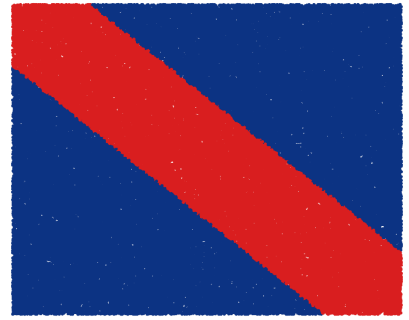

Source $1+$ Source $2+$ Source 3

Figure 6.5: Case Study 1: Synthetic two dimensional dataset with 3 sensors (sources), a ground truth, and fusion results. Red color coded samples have a value of 0 and blue indicates 1 . The answer is the minimum of sources 1 and 3 .

\subsection{CASE STUDIES}

\subsubsection{Synthetic: Multi-Sensor Fusion}

Numerous applications like remote sensing, smart cars, IoT, and beyond require the intelligent fusion of sensor data. Examples we have worked on include combining sensors across the electromagnetic spectrum on hand-held and ground-based platforms for humanitarian demining [100] and detecting human clandestine remains from drones with different sensor payloads [101]. In areas like computer vision, the community has built benchmark datasets for algorithmic validation and comparison. Sadly, there is not a widely accepted benchmark for fusion. As such, we use synthetic experiments to validate. Case Study 1 follows an experiment outlined in 34, 102, where we fused algorithms and sensor data in electromagnetic induction and hyperspectral image processing (see Fig. 6.5).

In Fig. 6.5, there are three sensors (sources) in a $[0,1]^{2}$ space. The data points 
are uniformly sampled across the space. Each sensor provides a 0 (colored red in Fig. 6.5) or 1 (colored blue) at each sample. As discussed in 34, 102, these samples can correspond to sensor inputs or algorithm outputs at spatial locations, e.g., a drone with a nadir sensor look angle. In Case Study 1, the answer (ground truth) is the minimum of two sensors, source 1 and source 3 . Source 2 is not needed. The fact that the inputs are binary is arbitrary; we did this to mimic fusion of binary classifiers (e.g., target or not target).

In Case Study 1, we first provide the user with sources 1 and 2 and a ChI is learned using quadratic programming [21]. Fig. 6.6 shows the resulting XAI fusion report. Overall, there are no anomalies or oddities. The top rank ordered XAI explanations show that all walks and variables are supported by data. The Shapley also shows that there are no useless sources. However, the accuracy of the classifier is poor, below 70\%. Accordingly, the report recommends that the user add a new source to improve accuracy 9

To show how a user may act on this report, we assume that the user follows the recommendation, adding a new source (source 3) and retraining the ChI. A new report is generated, shown in Fig. 6.7. Beyond the user observing that performance is now perfect, the report continues to provide potentially useful information. Namely, while source 2 provided benefit before, it is no longer needed with respect to the new added source. This can be of benefit on domains where sensors are expensive or have other costs, such as increased sensor payload weight and therefore lower flight time for an aerial platform. The point is, Case Study 1 shows that the report can be used in an interactive fashion until the user is content with the performance and the inner workings of the underlying process.

For sake of article length, we do not show expansion of these reports. However, each report section-linguistic explanations, visual explanations, etc.-can be

\footnotetext{
${ }^{9}$ With associated caveats for that action and disclaimers related to the machine learning of the integral.
} 


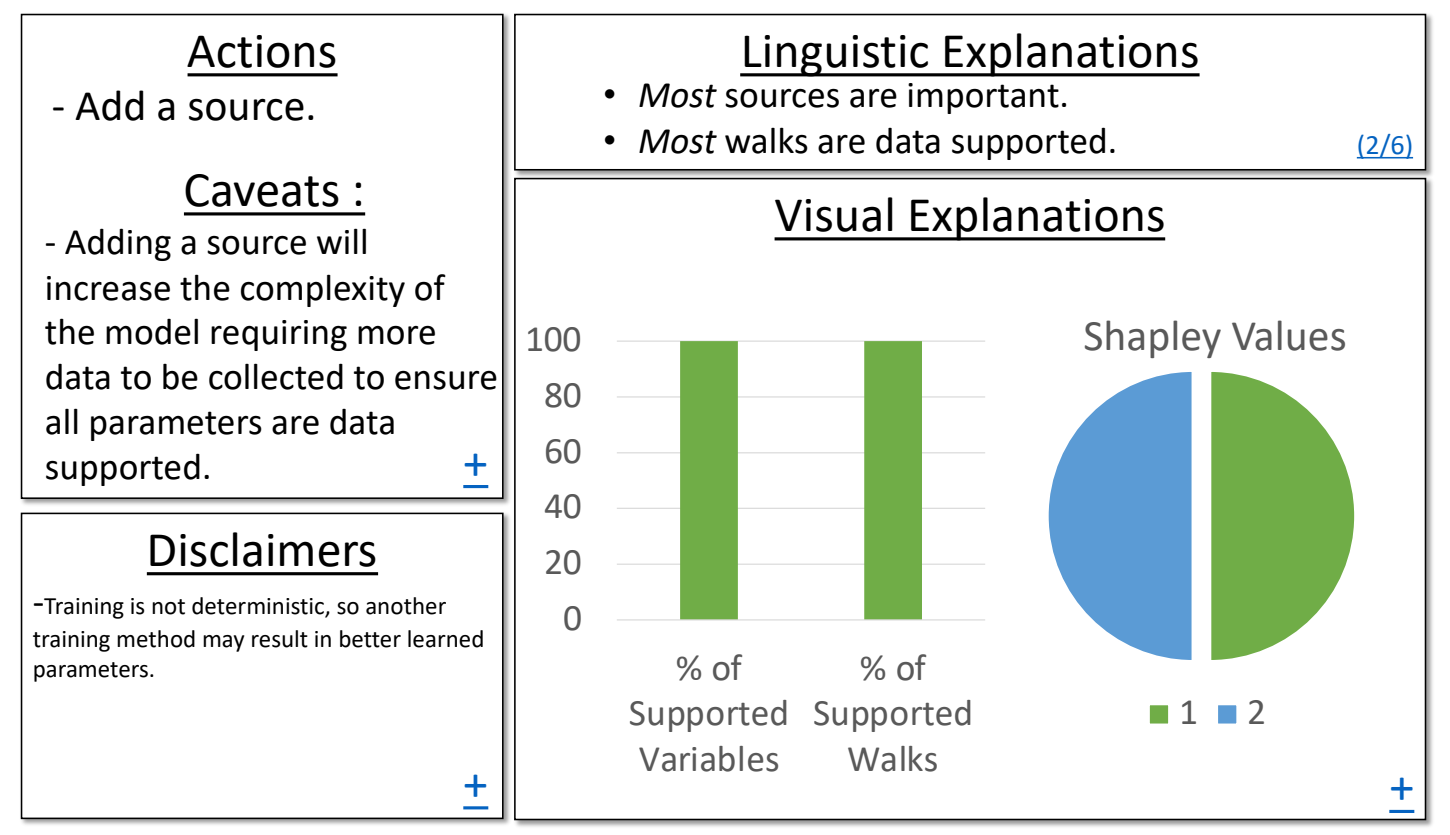

Figure 6.6: Case Study 1: Example fusion report recommending add a source. This action is supported by a small set of rank ordered low-level XAI explanations (right column).

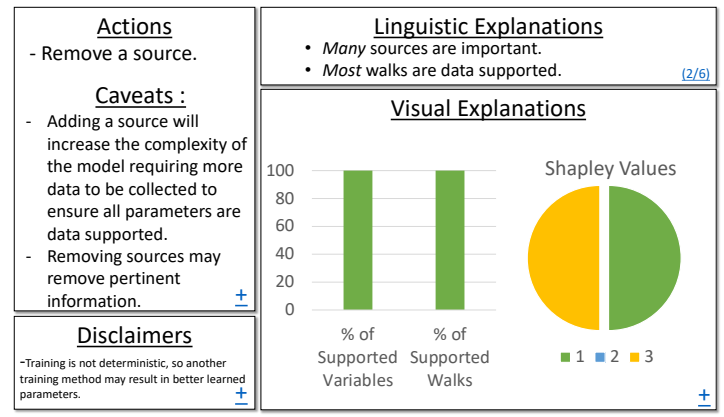

Figure 6.7: Case Study 1: Report showing that a source can be removed and no irregularities or anomalies otherwise exist. 
expanded (hyperlink in the lower right of each user interface box) as needed. Our philosophy is this is good for record keeping and as a chain of evidence, but it is not what a novice would perhaps use.

\subsubsection{Real-World: Deep Learning for Remote Sensing}

The next case study examines the fusion of multiple deep learning algorithms, where no single algorithm, architecture, or model is superior across datasets and classes. Specifically, in 54 we showed state-of-the-art results for land classification and object detection from satellite imagery for remote sensing across numerous benchmark community datasets based on the fusion of heterogeneous deep convolutional neural networks (DCNNs). Here, we focus on a single experiment, the AID remote sensing data set and seven DCNNs: CaffeNet [103], GoogleNet [104], ResNet 50 [72], ResNet 101, DenseNet [73], InceptionResNetV2 [74], and Xception [75]. The goal of this case study is to use the XAI report, not to validate the fusion of deep learners; that was already achieved in [54]. Fig. 6.8 is the result for a randomly selected cross validation fold.

There is no ground truth for Case Study 2 other than the labels of the training data; i.e., we do not know what is the best combination of DCNNs. Based on the report in Fig. 6.8, we explore two recommended actions.

The first action is based on the number of variables seen (over 80\%), but with very few of the possible walks (contexts) observed. This gives us reason to worry about model generalizability outside this particular data set, leading to a suggestion to acquire new data. Since these are benchmark data, we cannot collect more data, rendering this action obsolete ${ }^{11}$ If a goal was to deploy this model in a real-world setting - e.g., rapid broad area search and detection from remote sensing [105] - then

\footnotetext{
${ }^{10}$ The reader can refer to 54 for full details about the data sets, training, parameters, fusion specifics, etc.

${ }^{11}$ However, we do acknowledge that other potential remediations exist, e.g., identifying another data set to include in training.
} 


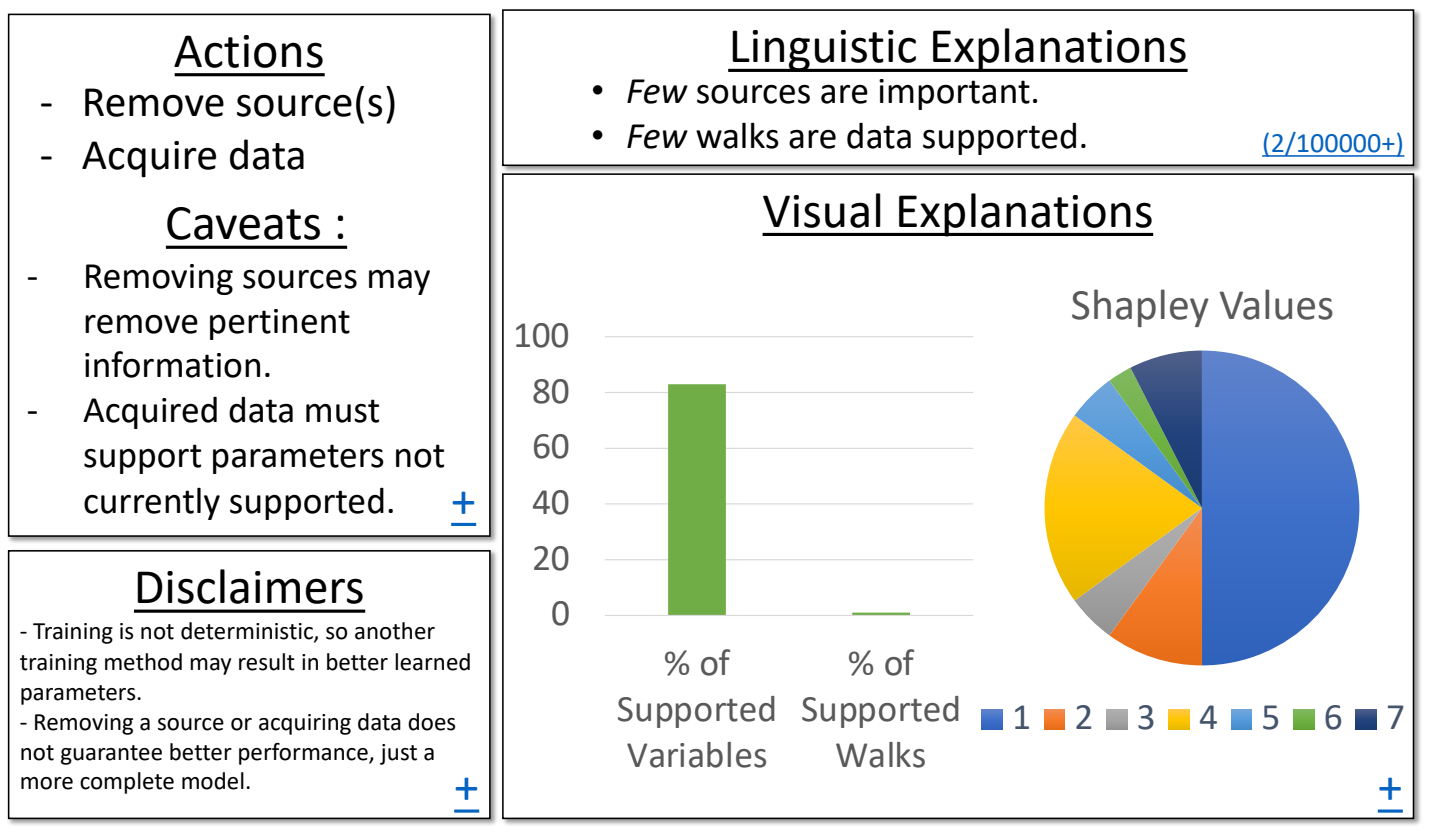

Figure 6.8: Case Study 2: The recommended actions are to remove sources and acquire more data. The visual explanations show that the model was supported by an extremely low amount of training data and there are many Shapley values indicating that sources could likely be removed.

this action could be revisited. In that situation, Fig. 6.9 shows a user clicking on the linguistic explanations section to obtain a list of ascending rank order probabilities for what specific data to collect.

The second recommended action involves our ability to choose the inputs (deep learning classifiers). As such, we examined the Shapley values and experimented with removing sources. Table 6.1 shows the impact of removing sources relative to accuracy. On a side note, we have not folded performance into the fusion report. A future scope is to extend the report into a more inclusive overall dashboard. Table 6.1 shows that we can remove a number of our sources without significant impact to the fusion accuracy, also improving the walk probability observation rates. With respect to the application of fusing deep learners, removing sources has benefits: fewer deep architectures to evaluate for a real-time system, less memory/storage, which ultimately lowers the complexity and cost of the hardware needed. 


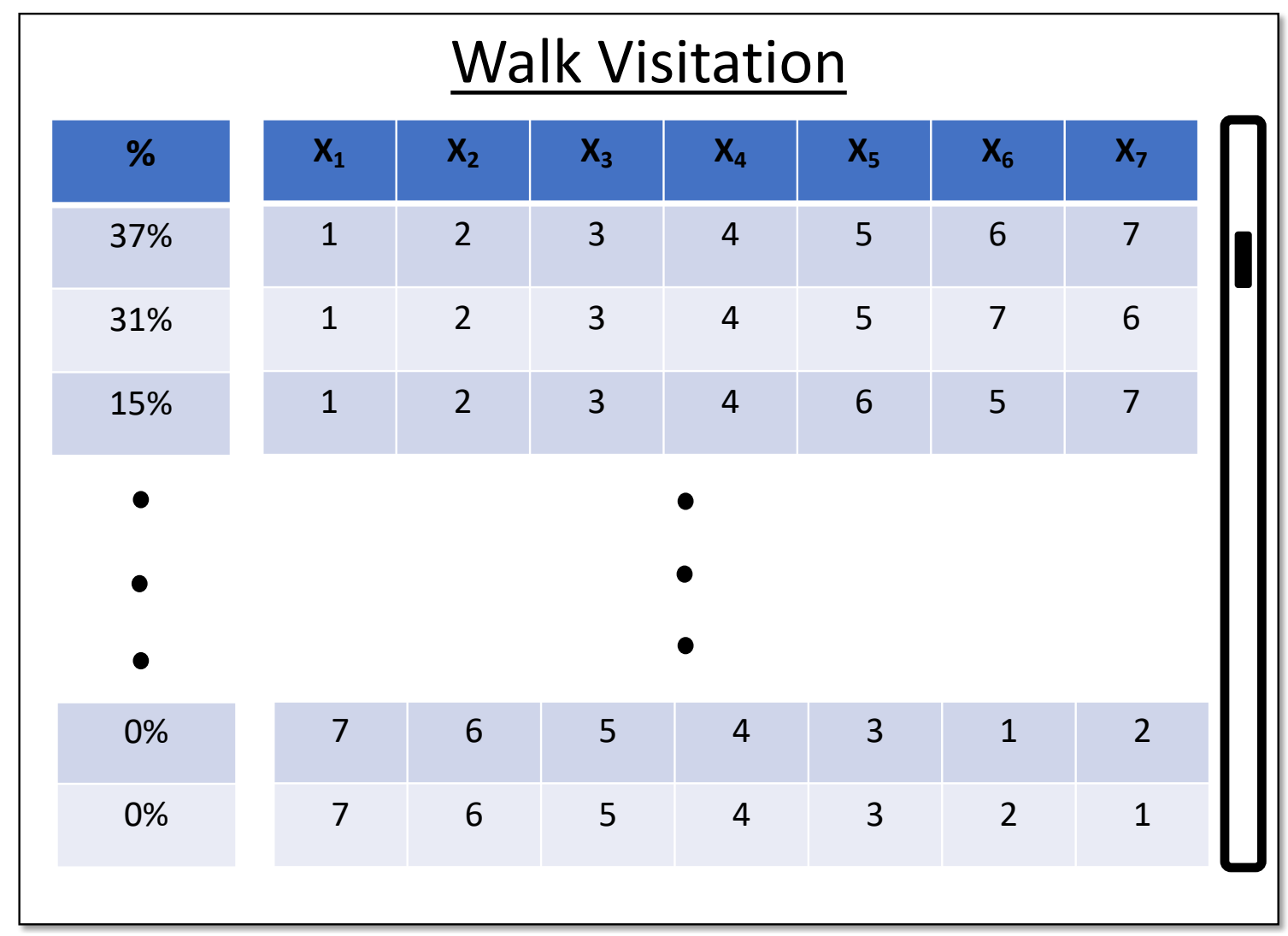

Figure 6.9: Case Study 2: More in-depth interface showing a rank ordering-by ascending probability-on walks (context). Context with little-to-no probability of observation require attention and additional data collection.

Table 6.1: Shapley values, percentage of walks observed, and accuracy of model when removing lowest valued source iteratively.

\begin{tabular}{lllllllll}
\hline Shapley & Shapley & Shapley & Shapley & Shapley & Shapley & Shapley & Walks & \\
1 & 2 & 3 & 4 & 5 & 6 & 7 & Observed & \\
\hline 0.43 & 0.01 & 0.01 & 0.04 & 0.01 & 0.27 & 0.23 & 0.01 & 0.992 \\
0.40 & 0.01 & 0.01 & .04 & $\mathrm{x}$ & 0.30 & 0.24 & 0.07 & 0.992 \\
0.39 & $\mathrm{x}$ & 0.01 & 0.08 & $\mathrm{x}$ & 0.29 & 0.23 & 0.36 & 0.992 \\
0.37 & $\mathrm{x}$ & $\mathrm{x}$ & 0.17 & $\mathrm{x}$ & 0.27 & 0.17 & 0.83 & 0.992 \\
0.2 & $\mathrm{x}$ & $\mathrm{x}$ & 0.15 & $\mathrm{x}$ & 0.65 & $\mathrm{x}$ & 1.0 & 0.992 \\
0.50 & $\mathrm{x}$ & $\mathrm{x}$ & $\mathrm{x}$ & $\mathrm{x}$ & 0.50 & $\mathrm{x}$ & 1.0 & 0.992 \\
1.0 & $\mathrm{x}$ & $\mathrm{x}$ & $\mathrm{x}$ & $\mathrm{x}$ & $\mathrm{x}$ & $\mathrm{x}$ & 1.0 & 0.99 \\
\hline
\end{tabular}


To summarize these case studies, Case Study 1 showed us identifying, understanding, and interactively resolving a fusion of binary classifiers task. In Case Study 2, which is exploratory as we do not know the answer (ground truth), the acquire new data action was deemed not possible and the remove source action helped to realize a simpler fusion solution with little loss in performance (accuracy).

\subsection{CONCLUSION AND FUTURE WORK}

In this article, we reviewed prior low-level XAI techniques for the ChI and we organized them according to where and how they fit into current XAI terminology. A limitation of prior ChI XAI tools is that they are informative but open ended. Herein, we explored the design of a fusion XAI report. The goal was to go beyond informing by recommending a set of actions that a user can take to overcome identified shortcomings. The report was designed to be user friendly to a novice. As such, only a handful of linguistic, statistical, and graphical trends and anomalies are reported. If and when a user wants or needs to dive deeper, the report allows for dynamic expansion and access to the full set of fusion XAI explanations. Two case studies, one synthetic and one an open ended real-world problem, were presented. The case studies highlighted the initial set of proposed actions relative to scenarios that we can control and act on, and scenarios where we cannot. Last, software for the methods proposed here are provided open source at https://github.com/B-Mur/ChoquetIntegral.git.

In future work, we plan to address the following. First, this article only begins to explore an interactive process between man and machine. We plan to more formally study what information is ideal to report and when to report it, relative to a specific task like improving a performance metric. Furthermore, the interface discussed here is only a prototype. We will expand it into more of a dashboard, like TensorFlow's TensorBoard. The idea is to improve the interface by integrating additional data

and reporting of relevant information like training statistics, algorithm performance, 
and whatever else is needed to help a user. Another future work includes identifying more atomic fusion actions. Furthermore, whereas we focused on human-machine interaction, we do not count out the possibility of closing the loop and using our action recommendation to perhaps automatically improve algorithms, e.g., machine learning automation. Last, higher-level compound linguistic descriptions likely need to be generated, our rank ordering of a handful of explanations could be improved, and existing XAI methods like local explanations and our trust index have yet to be incorporated relative to a user taking an action. 


\section{Chapter 7}

\section{CONCLUSION}

"Standing on the shoulders of giants" has never been more true than considering how the explainable data fusion has been realized. Looking back on this journey, we can appreciate the characteristics of the Choquet Integral. The pioneers of the integral proved its legitimacy and significance when applying to a variety of problems ranging from pattern recognition to multi-criteria decision making. Without these works setting the stage, it is unrealistic to believe that the XAI developed herein would even be possible. The innovative thinking that led to data driven learning of the Choquet Integral set off a chain reaction that allowed for the integral to become even more practical. Regardless of how you learn the FM parameters, the data has the same impact. Meaning, it allows for us to isolate how each variable directly contributes to not only the model but also each individual sample. This is what separates this data driven fusion operator from black box methods - the natural interpretability.

To date, no one has exploited the property of the natural interpretability as thoroughly as the XAI of this dissertation. The largest hurdle in this process was identifying and connecting to this very important characteristic to higher level meaning. Once this was recognized, explainability became about translating data driven implications to a practical form to generate low and higher level insights to both fusion experts and beginners. Through this process, a set of indices were developed that shine light 
on the fusion that show different views of the integral. For instance, highlighting the completeness of a learned model is an important answer when asking "how good is a model?" Another insightful index identifies the worth of each of the sources. While accumulating each of these data points provides context to a fusion expert, they are lacking when translating to a fusion amateur much less a fusion beginner.

An important component of research is answering the question "is this important an important endeavor?". Meaning, is the explainable data fusion an important research topic. In order to do this, receiving feedback is a critical step in answering this question. The last few chapters of this dissertation were influenced by the feedback received relative to the explainable data fusion indices. Understanding the lack of practicality of the low level indices propelled a higher level set of tools that provide actionable insights in a succinct fusion report. The goal of this dissertation is to provide an entire picture to the explainable data fusion, and delivering the fusion report is an effective tool to provide every fine detail of explainable.

While this dissertation has taken a deep dive into different components of the explainable data fusion (statistics, visualizations, summaries, reporting), each of these topics could be further extended. Specifically, each of the chapters is a thorough analysis of its subset of XAI, however each can also be further extended.

For instance, it may be relevant to an end user to not only understand that a FM parameter is or is not supported by data, but also how data supported a parameter is. Furthermore, model performance is generally gauged on accuracy of the output, however exploiting the XAI, we can isolate the accuracy of each specific walk - which allows for further targeting how to improve the model. Meaning, if one walk has low accuracy, we can study why this walk is lacking. Each of these examples highlights how more statistics can be generated that answer questions related to different aspects of the ChI. Granted, this is not the only area that can be extended.

Communicating these insights to an end user may be a cumbersome process. With 
so many statistics, we reduced them to a few summaries that describe the statistics and this the model. However, these summaries may be unrelated or even provide a minimal set of insights. However, by extending the summaries beyond the simple protoform, it is possible to produce summaries that better capture the entire set of insights that the statistics produce. Specifically, one avenue that can be taken is to perform an analysis of the generated summaries and to remove any redundant information. For example, if we know that only few sources are important, it is not valuable to provide a summary for each of the sources because we know that only a few are important. Considering the relationships among the summaries, we are able to provide a targeted approach to which summaries to provide (rather than just providing the ones with the highest truth values).

Lastly, generating a fusion report is a pivotal part of the story of the ChI. Making the ChI more accessible will require providing higher level tools that a fusion beginner can use. There is an art to not generating all the information to the end user; rather, it takes deep understanding of the end user and ChI to provide the right information. It is likely that this information may be application dependent. Further analysis can be conducted to determine the appropriate across different applications.

This dissertation captures explainable data fusion relative to a single fusion operator; however, questions surrounding the philosophy of how fusion (and AI in general) should be explained still exist. The goal of this dissertation was not to address these question; rather, these questions were exposed throughout this work. This work effectively answered how explanations can be generated for fusion, but the remaining questions ask why explanations are necessary. Mathematics are the pieces of the XAI puzzle. It is not only up to the human to assemble the puzzle, but also to give meaning to the picture. To a lover of the outdoors, a puzzle that pictures a beautiful landscape will convey much more meaning than random lines and shapes. Similarly, in the right context XAI can paint a beautiful picture, but it can also be a series of 
random pieces of meaningless information. Moreover, the current state of AI requires humans be involved at some capacity. However, one goal of XAI may be to inform itself. In the context of the XAI presented herein, the system can recognize when there are sources that are not important, and it can be automate to remove those sources. This form of reasoning is typically left to the fusion context, but as the algorithms mature, this type of reasoning should be included.

As data grows, it is becoming increasingly hard for humans to recognize patterns and mentally optimize systems. However, turning these decisions over to the algorithms should allow for algorithms with less bias than humans. Explanations are one way for the algorithms to communicate with humans. Understanding how we reason with explanations drives our innovation in bettering our algorithms. This is not a new concept, but it becomes increasingly important. This idea extends beyond data fusion, but understanding the future of algorithms should determine the decisions that are made today. This is why explanations are so important. The XAI that is constructed today is affecting the decisions that AI will be making tomorrow. The AI we are making tomorrow will quite possibly drive society as we know it. 


\section{BIBLIOGRAPHY}

[1] J. L. Parker. Driverless Cars Gain Speed, Despite Global Slowdown. URL: https : / / www . forbes . com/sites / jenniferleighparker / 2020 / 08 / 07 / driverless-cars-gain-speed-despite-global-slowdown/\#414c56d82e86.

[2] K. Buhler. 3 Ways Artificial Intelligence Will Change Healthcare. URL: https: // www . forbes . com / sites / konstantinebuhler / 2020 / 08 / 04 / 3-ways artificial-intelligence-will-change-healthcare/\#1e2cc433e4ed.

[3] National Artificial Intelligence (AI) Research Institutes Accelerating Research, Transforming Society, and Growing the American Workforce. URL: https : //www.nsf.gov/pubs/2020/nsf20503/nsf20503.htm.

[4] A. Jobin, M. Ienca, and E. Vayena. The global landscape of AI ethics guidelines. Nature Machine Intelligence, 1:389-399, 2019.

[5] N. Mehrabi, F. Morstatter, N. Saxena, K. Lerman, and A. Galstyan. A Survey on Bias and Fairness in Machine Learning, 2019. arXiv: 1908.09635 [cs.LG].

[6] M. Turek. Explainable Artificial Intelligence (XAI).

[7] M. D. Zeiler, D. Krishnan, G. W. Taylor, and R. Fergus. Deconvolutional networks:2528-2535, 2010.

[8] M. D. Zeiler, G. W. Taylor, and R. Fergus. Adaptive deconvolutional networks for mid and high level feature learning:2018-2025, 2011. 
[9] K. Simonyan, A. Vedaldi, and A. Zisserman. Deep Inside Convolutional Networks: Visualising Image Classification Models and Saliency Maps, 2013. arXiv: 1312.6034 [cs.CV].

[10] A. Mahendran and A. Vedaldi. Understanding Deep Image Representations by Inverting Them, 2014. arXiv: 1412.0035 [cs.CV].

[11] M. T. Ribeiro, S. Singh, and C. Guestrin. "Why Should I Trust You?": Explaining the Predictions of Any Classifier. CoRR, abs/1602.04938, 2016. arXiv: 1602.04938. URL: http://arxiv.org/abs/1602.04938.

[12] S. Lundberg and S.-I. Lee. A Unified Approach to Interpreting Model Predictions, 2017. arXiv: 1705.07874 [cs.AI].

[13] S. Vasudevan and K. Kenthapadi. LiFT: A Scalable Framework for Measuring Fairness in ML Applications. CIKM '20, 2020.

[14] J. A. Sanz, D. Bernardo, F. Herrera, H. Bustince, and H. Hagras. A Compact Evolutionary Interval-Valued Fuzzy Rule-Based Classification System for the Modeling and Prediction of Real-World Financial Applications With Imbalanced Data. IEEE Transactions on Fuzzy Systems, 23(4):973-990, 2015.

[15] H. Hagras. Toward Human-Understandable, Explainable AI, 2018.

[16] D. Pekaslan, C. Chen, C. Wagner, and J. M. Garibaldi. Performance and Interpretability in Fuzzy Logic Systems - Can We Have Both?:571-584, 2020. Ed. by M.-J. Lesot, S. Vieira, M. Z. Reformat, J. P. Carvalho, A. Wilbik, B. Bouchon-Meunier, and R. R. Yager.

[17] J. .--. R. Jang. ANFIS: adaptive-network-based fuzzy inference system. IEEE Transactions on Systems, Man, and Cybernetics, 23(3):665-685, 1993.

[18] D. Pekaslan, C. Wagner, and J. M. Garibaldi. ADONiS - Adaptive Online Non-Singleton Fuzzy Logic Systems. IEEE Transactions on Fuzzy Systems:11, 2019. 
[19] A. Adadi and M. Berrada. Peeking Inside the Black-Box: A Survey on Explainable Artificial Intelligence (XAI). IEEE Access, 6:52138-52160, 2018.

[20] F. K. Dovsilović, M. Brvcić, and N. Hlupić. Explainable artificial intelligence: A survey:0210-0215, May 2018.

[21] M. A. Islam, D. T. Anderson, A. J. Pinar, and T. C. Havens. Data-Driven Compression and Efficient Learning of the Choquet Integral. IEEE Transactions on Fuzzy Systems, PP(99):1-1, 2017. ISSN: 1063-6706.

[22] R. E. Smith, D. T. Anderson, A. Zare, J. E. Ball, B. Alvey, J. R. Fairley, and S. E. Howington. Genetic Programming Based Choquet Integral for MultiSource Fusion, July 2017.

[23] P. D. Gader, J. M. Keller, and B. N. Nelson. Recognition technology for the detection of buried land mines, 9(1):31-43, 2001.

[24] H. Tahani and J. Keller. Information fusion in computer vision using the fuzzy integral. IEEE Transactions System, Man, and Cybernetics, 20:733-741, 1990.

[25] M. Grabisch and J. Nicolas. Classification by fuzzy integral: Performance and tests. Fuzzy Sets $\mathcal{G}$ Sys., 65(2-3):255-271, 1994.

[26] A. Mendez-Vazquez, P. Gader, J. M. Keller, and K. Chamberlin. Minimum Classification Error Training for Choquet Integrals With Applications to Landmine Detection. IEEE Transactions on Fuzzy Systems, 16(1):225-238, Feb. 2008. ISSN: 1063-6706.

[27] J. M. Keller, P. Gader, H. Tahani, J. Chiang, and M. Mohamed. Advances in fuzzy integration for pattern recognition. Fuzzy Sets and Systems, 65(2-3):273283, 1994.

[28] M. Grabisch. The application of fuzzy integrals in multicriteria decision making. European Journal of Operational Research, 89(3):445-456, 1996. 
[29] C. Labreuche. Construction of a Choquet integral and the value functions without any commensurateness assumption in multi-criteria decision making:9097, 2011.

[30] D. T. Anderson, P. Elmore, F. Petry, and T. C. Havens. Fuzzy Choquet integration of homogeneous possibility and probability distributions. Information Sciences, 363:24-39, 2016. ISSN: 0020-0255. URL: http://www. sciencedirect. com/science/article/pii/S0020025516302961.

[31] M. Anderson, D. T. Anderson, and D. J. Wescott. Estimation of adult skeletal age-at-death using the Sugeno fuzzy integral. American Journal of Physical Anthropology, 142(1):30-41, 2010.

[32] L. Tomlin, D. T. Anderson, C. Wagner, T. C. Havens, and J. M. Keller. Fuzzy Integral for Rule Aggregation in Fuzzy Inference Systems. Springer International Publishing, 2016, 78-90.

[33] A. J. Pinar, J. Rice, L. Hu, D. T. Anderson, and T. C. Havens. Efficient Multiple Kernel Classification using Feature and Decision Level Fusion, PP(99):1-1, 2016. ISSN: 1063-6706.

[34] X. Du, A. Zare, J. M. Keller, and D. T. Anderson. Multiple Instance Choquet integral for classifier fusion:1054-1061, July 2016.

[35] M. Al Boni, D. T. Anderson, and R. L. King. Hybrid Measure of Agreement and Expertise for Ontology Matching in Lieu of a Reference Ontology. International Journal of Intelligent Systems, 31(5):502-525, 2016. ISSN: 1098-111X.

[36] M. A. Islam, D. T. Anderson, F. Petry, D. Smith, and P. Elmore. The fuzzy integral for missing data:1-8, July 2017.

[37] D. Anderson, G. Scott, M. Islam, B. Murray, and R. Marcum. Fuzzy Choquet Integration of Deep Convolutional Neural Networks for Remote Sensing:pppp, 2018. Ed. by Witold and Shyi-Ming. 
[38] G. J. Scott, R. A. Marcum, C. H. Davis, and T. W. Nivin. Fusion of Deep Convolutional Neural Networks for Land Cover Classification of High-Resolution Imagery. IEEE Geoscience $\& 3$ Remote Sens. Letters, 2017.

[39] M. Sugeno. Theory of fuzzy integrals and its applications. Ph.D. thesis, Tokyo Institute of Technology, 1974.

[40] A. J. Pinar, D. T. Anderson, T. C. Havens, A. Zare, and T. Adeyeba. Measures of the Shapley index for learning lower complexity fuzzy integrals. Granular Computing:1-17, 2017.

[41] D. T. Anderson, S. R. Price, and T. C. Havens. Regularization-based learning of the Choquet integral:2519-2526, July 2014. ISSN: 1098-7584.

[42] T. Murofushi and S. Soneda. Techniques for reading fuzzy measures (III): interaction index:693-696, 1993.

[43] M. Grabisch and M. Roubens. An axiomatic approach to the concept of interaction among players in cooperative games. International Journal of Game Theory, 28(4):547-565, 1999.

[44] M. Grabisch. An axiomatization of the Shapley value and interaction index for games on lattices, 2004.

[45] S. R. Price, D. T. Anderson, C. Wagner, T. C. Havens, and J. M. Keller. Indices for Introspection on the Choquet Integral:261-271, 2014.

[46] G. J. Scott, M. R. England, W. A. Starms, R. A. Marcum, and C. H. Davis. Training Deep Convolutional Neural Networks for Land-Cover Classification of High-Resolution Imagery. IEEE Geoscience and Remote Sensing Letters, 14(4):549-553, 2017.

[47] L. Ductu, G. Mauris, and P. Bolon. A Fast and Accurate Rule-Base Generation Method for Mamdani Fuzzy Systems. IEEE Transactions on Fuzzy Systems, 26(2):715-733, Apr. 2018. Issn: 1063-6706. 
[48] O. Cordon, F. Herrera, and P. Villar. Generating the knowledge base of a fuzzy rule-based system by the genetic learning of the data base. IEEE Transactions on Fuzzy Systems, 9(4):667-674, Aug. 2001. ISSN: 1063-6706.

[49] A. Jain and J. M. Keller. Textual summarization of events leading to health alerts:7634-7637, Aug. 2015. ISSN: 1094-687X.

[50] G. Smits, P. Nerzic, O. Pivert, and M.-J. Lesot. Efficient Generation of Reliable Estimated Linguistic Summaries, July 2018. URL: https://hal .archivesouvertes.fr/hal-01854298.

[51] M. D. Zeiler and R. Fergus. Visualizing and understanding convolutional networks:818-833, 2014.

[52] Q.-s. Zhang and S.-c. Zhu. Visual interpretability for deep learning: a survey. Frontiers of Information Technology Electronic Engineering, 19:27-39, Jan. 2018.

[53] X. Du and A. Zare. Multiple Instance Choquet Integral Classifier Fusion and Regression for Remote Sensing Applications. IEEE Transactions on Geoscience and Remote Sensing:1-13, 2018. ISSN: 0196-2892.

[54] G. J. Scott, K. C. Hagan, R. A. Marcum, J. A. Hurt, D. T. Anderson, and C. H. Davis. Enhanced Fusion of Deep Neural Networks for Classification of Benchmark High-Resolution Image Data Sets. IEEE Geoscience and Remote Sensing Letters, 15(9):1451-1455, Sept. 2018. ISSN: 1545-598X.

[55] G. Choquet. Theory of capacities. In: Annales de l'institut Fourier. Vol. 5. Institut Fourier. 1954, 131-295.

[56] M. Sugeno and T. Murofushi. Pseudo-additive measures and integrals. Journal of Mathematical Analysis and Applications, 122:197222, 1987. 
[57] T. C. Havens, A. J. Pinar, D. T. Anderson, and C. Wagner. SPFI: ShapePreserving Choquet Fuzzy Integral for Non-Normal Fuzzy Set-Valued Evidence. In: 2018 IEEE International Conference on Fuzzy Systems (FUZZIEEE). July 2018, 1-6.

[58] D. T. Anderson, T. C. Havens, C. Wagner, J. M. Keller, M. F. Anderson, and D. J. Wescott. Extension of the Fuzzy Integral for General Fuzzy Set-Valued Information, 22(6):1625-1639, 2014. ISSN: 1063-6706.

[59] N. Shilkret. Maxitive measure and integration. Indagationes Mathematicae (Proceedings), 74:109-116, 1971. ISSN: 1385-7258. URL: http://www. sciencedirect. com/science/article/pii/S1385725871800173.

[60] M. A. Islam, D. Anderson, F. Petry, and P. Elmore. An Efficient Evolutionary Algorithm to Optimize the Choquet Integral. International Journal of Intelligent Systems, Sept. 2018.

[61] M. A. Islam, D. T. Anderson, A. Pinar, T. C. Havens, G. Scott, and J. M. Keller. Enabling Explainable Fusion in Deep Learning with Fuzzy Integral Neural Networks. IEEE Transactions on Fuzzy Systems:1-1, 2019. ISSN: 19410034. URL: http://dx.doi.org/10.1109/TFUZZ.2019.2917124.

[62] J. M. Keller and J. Osborn. Training the fuzzy integral. International Journal of Approximate Reasoning, 15(1):1-24, 1996.

[63] J. M. Keller and J. Osborn. A reward/punishment scheme to learn fuzzy densities for the fuzzy integral. Proc. Int. Fuzzy Sys. Assoc. World Cong:97-100, 1995.

[64] G. Beliakov. Construction of aggregation functions from data using linear programming. Fuzzy Sets and Systems, 160(1):65-75, 2009. 
[65] S. Angilella, S. Corrente, and S. Greco. Stochastic Multiobjective Acceptability Analysis for the Choquet integral preference model and the scale construction problem. European Journal of Operational Research, 240:172-182, Jan. 2015.

[66] J. Marichal and M. Roubens. Determination of weights of interacting criteria from a reference set. European Journal of Operational Research, 124:641-650.

[67] S. Angilella, S. Greco, and B. Matarazzo. Non-additive robust ordinal regression: A multiple criteria decision model based on the Choquet integral. European Journal of Operational Research, 201:277-288.

[68] L. S. Shapley. A value for n-person games. Contributions to the Theory of Games, 2:307-317, 1953.

[69] M. Grabisch and M. Roubens. Application of the Choquet integral in multicriteria decision making:348-374, 2000.

[70] Y. Jia, E. Shelhamer, J. Donahue, S. Karayev, J. Long, R. Girshick, S. Guadarrama, and T. Darrell. Caffe: Convolutional Architecture for Fast Feature Embedding. MM '14:675-678, 2014. URL: http :// doi . acm.org/10.1145/ 2647868.2654889 .

[71] C. Szegedy, W. Liu, Y. Jia, P. Sermanet, S. Reed, D. Anguelov, D. Erhan, V. Vanhoucke, and A. Rabinovich. Going Deeper with Convolutions, 2015. URL: http://arxiv.org/abs/1409.4842.

[72] K. He, X. Zhang, S. Ren, and J. Sun. Deep Residual Learning for Image Recognition. arXiv preprint arXiv:1512.03385, 2015.

[73] G. Huang, Z. Liu, L. v. d. Maaten, and K. Q. Weinberger. Densely Connected Convolutional Networks:2261-2269, July 2017. ISSN: 1063-6919.

[74] C. Szegedy, S. Ioffe, and V. Vanhoucke. Inception-v4, Inception-ResNet and the Impact of Residual Connections on Learning, 2016. 
[75] F. Chollet. Xception: Deep Learning with Depthwise Separable Convolutions:18001807, July 2017. ISSN: 1063-6919.

[76] G. Xia, J. Hu, F. Hu, B. Shi, X. Bai, Y. Zhong, L. Zhang, and X. Lu. AID: A Benchmark Data Set for Performance Evaluation of Aerial Scene Classification. IEEE Transactions on Geoscience and Remote Sensing, 55(7):3965-3981, 2017.

[77] B. J. Murray, M. J. Islam, A. J. Pinar, D. T. Anderson, G. J. Scott, T. C. Havens, and J. M. Keller. Explainable AI for the Choquet Integral (under review). IEEE Transactions on Emerging Topics in Computational Intelligence.

[78] B. Murray, D. Anderson, M. A. Islam, A. Pinar, G. Scott, and T. Havens. Explainable AI for Understanding Decisions and Data-Driven Optimization of the Choquet Integral. In: World Congress on Computational Intelligence (WCCI). July 2018.

[79] J. Kacprzyk, A. Wilbik, and S. Zadrozny. Mining time series data via linguistic summaries of trends by using a modified Sugeno integral based aggregation. In: 2007 IEEE Symposium on Computational Intelligence and Data Mining. Mar. 2007, 742-749.

[80] A. Wilbik, J. M. Keller, and J. C. Bezdek. Linguistic Prototypes for Data From Eldercare Residents. IEEE Transactions on Fuzzy Systems, 22(1):110123, Feb. 2014. ISSN: 1941-0034.

[81] J. Kacprzyk and S. Zadrozny. Data mining via protoform based linguistic summaries: Some possible relations to natural language generation. In: 2009 IEEE Symposium on Computational Intelligence and Data Mining. Mar. 2009, $217-224$.

[82] R. R. Yager. A new approach to the summarization of data. Information Sciences, 28(1):69-86, 1982. ISSN: 0020-0255. 
[83] R. R. Yager. Fuzzy summaries in database mining. In: Proceedings the 11th Conference on Artificial Intelligence for Applications. Feb. 1995, 265-269.

[84] A. Wilbik, U. Kaymakand, J. M. Keller, M. Popescu, P. Angelov, K. T. Atanassov, L. Doukovska, M. Hadjiski, V. Jotsov, and J. K. et al. On the computation of semantically ordered truth values of linguistic protoform summaries. In: Intelligent Systems. Vol. 322. Aug. 2014, 69-79.

[85] A. Jain and J. M. Keller. On the computation of semantically ordered truth values of linguistic protoform summaries. In: 2015 IEEE International Conference on Fuzzy Systems (FUZZ-IEEE). Aug. 2015, 1-8.

[86] M. Delgado, M. D. Ruiz, D. Sánchez, and M. A. Vila. Fuzzy quantification: a state of the art. Fuzzy Sets and Systems, 242:1-30, 2014. Theme: Quantifiers and Logic. ISSN: 0165-0114. URL: http://wWW.sciencedirect.com/science/ article/pii/S0165011413004247.

[87] S. J. Pan and Q. Yang. A Survey on Transfer Learning. IEEE Transactions on Knowledge and Data Engineering, 22(10):1345-1359, Oct. 2010. ISSN: 10414347 .

[88] L. Shao, F. Zhu, and X. Li. Transfer Learning for Visual Categorization: A Survey. IEEE Transactions on Neural Networks and Learning Systems, 26(5):1019-1034, May 2015. ISSN: 2162-237X.

[89] A. J. Pinar, T. C. Havens, M. A. Islam, and D. T. Anderson. Visualization and learning of the Choquet integral with limited training data. In: 2017 IEEE International Conference on Fuzzy Systems (FUZZ-IEEE). July 2017, 1-6.

[90] A. Barredo Arrieta, N. Díaz-Rodríguez, J. Del Ser, A. Bennetot, S. Tabik, A. Barbado, S. Garcia, S. Gil-Lopez, D. Molina, R. Benjamins, R. Chatila, and F. Herrera. Explainable Artificial Intelligence (XAI): Concepts, taxonomies, opportunities and challenges toward responsible AI. Information Fusion, 58:82117 
115, 2020. ISSN: 1566-2535. URL: http://wWW.sciencedirect.com/science/ article/pii/S1566253519308103.

[91] D. Pekaslan, C. Chen, C. Wagner, and J. M. Garibaldi. Performance and Interpretability in Fuzzy Logic Systems - Can We Have Both? In: Information Processing and Management of Uncertainty in Knowledge-Based Systems - 18th International Conference, IPMU 2020, Lisbon, Portugal, June 15-19, 2020, Proceedings, Part I. Ed. by M. Lesot, S. M. Vieira, M. Z. Reformat, J. P. Carvalho, A. Wilbik, B. Bouchon-Meunier, and R. R. Yager. Vol. 1237. Communications in Computer and Information Science. Springer, 2020, 571-584. URL: https://doi.org/10.1007/978-3-030-50146-4\%5C_42.

[92] R. R. Selvaraju, M. Cogswell, A. Das, R. Vedantam, D. Parikh, and D. Batra. Grad-CAM: Visual Explanations from Deep Networks via Gradient-Based Localization. International Journal of Computer Vision, 128(2):336-359, Oct. 2019. ISSN: 1573-1405. URL: http://dx.doi.org/10 .1007/s11263-01901228-7.

[93] B. Murray, M. A. Islam, A. J. Pinar, T. C. Havens, D. T. Anderson, and G. Scott. Explainable AI for Understanding Decisions and Data-Driven Optimization of the Choquet Integral. In: 2018 IEEE International Conference on Fuzzy Systems (FUZZ-IEEE). 2018, 1-8.

[94] B. J. Murray, M. A. Islam, A. J. Pinar, D. T. Anderson, G. J. Scott, T. C. Havens, and J. M. Keller. Explainable AI for the Choquet Integral. IEEE Transactions on Emerging Topics in Computational Intelligence:1-10, 2020.

[95] J. Adebayo, J. Gilmer, M. Muelly, I. Goodfellow, M. Hardt, and B. Kim. Sanity Checks for Saliency Maps. In: Advances in Neural Information Processing Systems 31. Ed. by S. Bengio, H. Wallach, H. Larochelle, K. Grauman, N. Cesa-Bianchi, and R. Garnett. Curran Associates, Inc., 2018, 9505-9515. URL: 
http://papers . nips.cc/paper/8160-sanity-checks-for-saliencymaps . pdf.

[96] A. J. Pinar, T. C. Havens, M. A. Islam, and D. T. Anderson. Visualization and learning of the Choquet integral with limited training data. In: 2017 IEEE International Conference on Fuzzy Systems (FUZZ-IEEE). 2017, 1-6.

[97] A. R. Buck, D. T. Anderson, J. M. Keller, T. Wilkin, and M. A. Islam. A Weighted Matrix Visualization for Fuzzy Measures and Integrals. In: 2020 IEEE International Conference on Fuzzy Systems (FUZZ-IEEE). 2020, 1-8.

[98] S. Kakula, A. Pinar, T. Havens, and D. Anderson. Visualization and Analysis Tools for Explainable Choquet Integral Regression. IEEE Symposium Series on Computational Intelligence, 2020.

[99] B. J. Murray, D. T. Anderson, T. C. Havens, T. Wilkin, and A. Wilbik. Information Fusion-2-Text: Explainable Aggregation via Linguistic Protoforms. In: Information Processing and Management of Uncertainty in Knowledge-Based Systems. Ed. by M.-J. Lesot, S. Vieira, M. Z. Reformat, J. P. Carvalho, A. Wilbik, B. Bouchon-Meunier, and R. R. Yager. Cham: Springer International Publishing, 2020, 114-127. ISBN: 978-3-030-50153-2.

[100] T. Havens, D. Anderson, K. Stone, J. Becker, and A. Pinar. Computational Intelligence Methods in Forward-Looking Explosive Hazard Detection, 621:1344, Oct. 2016.

[101] D. Anderson, B. Murray, D. Wescott, R. Moorhead, and M. Anderson. Survey and Insights into Unmanned Aerial Vehicle-Based Detection and Documentation of Clandestine Graves and Human Remains. Human Biology, 90, May 2018.

[102] X. Du, A. Zare, and D. T. Anderson. Multiple Instance Choquet Integral with Binary Fuzzy Measures for Remote Sensing Classifier Fusion with Impre119 
cise Labels. In: 2019 IEEE Symposium Series on Computational Intelligence (SSCI). 2019, 1154-1162.

[103] Y. Jia, E. Shelhamer, J. Donahue, S. Karayev, J. Long, R. Girshick, S. Guadarrama, and T. Darrell. Caffe: Convolutional architecture for fast feature embedding:675-678, 2014.

[104] C. Szegedy, W. Liu, Y. Jia, P. Sermanet, S. Reed, D. Anguelov, D. Erhan, V. Vanhoucke, and A. Rabinovich. Going deeper with convolutions:1-9, 2015.

[105] R. A. Marcum, C. H. Davis, G. J. Scott, and T. W. Nivin. Rapid broad area search and detection of Chinese surface-to-air missile sites using deep convolutional neural networks. Journal of Applied Remote Sensing, 11(4):1-31, 2017. URL: https://doi.org/10.1117/1.JRS.11.042614. 


\section{VITA}

Bryce J. Murray was born in the little river-town of Natchez, MS. Bryce spent most of his summers working with his grandfather on the family farm, which is where his imagination ran wild with the possibilities of things that could and should be built from all the scrap materials lying around the barn. It wasn't long before he went to Mississippi College in Clinton, MS where he earned his Bachelor's degree in Computer Science, Physics, and Mathematics. Upon graduation, Bryce decided to attend Mississippi State University in Starkville, MS where he completed his Master's degree in Electrical and Computer Engineering. MSU is where Bryce met Dr. Derek Anderson, and the reason he decided to come to the University of Missouri. 